\title{
RECENT PROGRESS AND FUTURE PROSPECTS IN SOLAR PHYSICS, AND THEIR RELEVANCE FOR PLANET EARTH
}

\author{
ROGER M. BONNET and ANUSCHKA PAULUHN* \\ International Space Science Institute, Hallerstrasse 6, 3012 Bern, Switzerland \\ *E-mail:pauluhn@issi.unibe.ch
}

(Received 14 June 2004; Accepted 25 November 2004)

\begin{abstract}
Understanding our star, the Sun, is of fundamental interest for life on Earth. In this paper, the status of knowledge in solar physics of roughly two decades ago is summarised, and the most recent developments in this very active field are shown, many of them achieved by means of space based missions. The Sun-Earth connection is described and, finally, an outlook on future solar research is given.
\end{abstract}

Keywords: Solar physics, ground-based measurements, space missions, Sun-Earth connection

\section{Introduction}

During the last 15 years new developments and instrumentation, especially in space missions, have led to significant progress in our knowledge and understanding of the Sun. The Sun is a very close main-sequence star of average mass, luminosity and effective temperature, a typical "cool star" of spectral class G2. Understanding the structure and the processes inside the Sun is mandatory for any kind of study of star formation, galaxy evolution or cosmological models. As the Sun is by far the closest star ( $\alpha$ Centauri is more than $10^{5}$ times farther away), it offers a unique possibility for observation, in particular its atmosphere can be studied in great detail, and spatially resolved observations can be performed. Thus, it is a unique laboratory for fundamental research in particle and atomic physics, magnetism, plasma physics and their mutual interplay. As such it can be considered as a reference star.

Ground-based observations of the Sun have always been compromised by the effects of the Earth's atmosphere, not only by turbulence leading to image distortion, but also by atmospheric extinction, which affects most parts of the radiative spectrum. The latter can only be overcome by observations from space. Since about 1950, numerous solar experiments have been carried out using balloons, aircraft, rockets and satellites. Artificial satellites offer the possibility to observe the Sun from unusual vantage points, as for example the Sun-Earth Lagrangian point $L_{1}$, or from outside the ecliptic plane, or in stereoscopic modes, using very different orbits.

Surveys in Geophysics 25: 371-440, 2004.

2004 Kluwer Academic Publishers. Printed in the Netherlands. 
In recent years, the Sun's interaction with the Earth's atmosphere has gained more and more attention. It has been realized that the effects of solar activity on the terrestrial climate system as well as on technological and biological systems are of vital importance. Various studies on the impact of the variation of the solar radiative and particle flux and of the solar irradiance have been performed, in view of their influence on the climate. The extreme ultraviolet (EUV) radiation has proven to be of particular importance for the Sun-Earth connection due to its effect on the Earth's middle and upper atmosphere. In addition, a fast growing area of research is developing in the field of "space weather" that refers to the state of the interplanetary medium (IPM) and of the Earth's magnetosphere and ionosphere influenced by the solar activity. During unusually high charged particle flux conditions ("storms"), terrestrial systems like communication networks, power grids, and satellites can suffer deleterious effects. Since such systems are more and more indispensable for our modern way of living, it becomes necessary to understand better the processes through which the Sun influences the Earth, and possibly to predict the manifestations of its activity.

This paper intends to review the present situation of solar physics with respect to our knowledge of 20 years ago. It will describe the dramatic jumps witnessed during this time span thanks to observations from space as well as from the ground made possible by innovative instruments. It will address the problem of Sun-Earth relations, in particular the recurrent question of the Sun's influence on our climate, and the space weather issue. It will offer a prospective view on what this very active branch of science will offer in terms of progress and future investigations.

\section{What did we know 20 years ago?}

\subsection{MODELS OF THE SOLAR INTERIOR: NEUTRINO DEFICIT AND HELIOSEISMOLOGY}

The basic theoretical model of the Sun's interior, whereby the energy is produced by nuclear fusion processes in the core, goes back to the thirties of last century (Bethe and Critchfield, 1938; Bethe, 1939). Together with a model of the structure of the Sun and the conditions in its centre, namely temperature, pressure, particle density, composition, the so-called standard model (Bahcall et al., 1995), the reaction chains through which the Sun transforms its hydrogen content into helium (proton-proton (pp) and carbon-nitrogen-oxygen (CNO) reactions) determine the luminous energy of the Sun (the total radiative output per unit time of the Sun is $3.84 \times 10^{26} \mathrm{~W}$ ), as well as an enormous flux of neutrinos (roughly $6.57 \times 10^{14} \mathrm{~m}^{-2} \mathrm{~s}^{-1}$ ), spread over a wide range of energy (about $0.1-$ 
$15 \mathrm{MeV}$ ). These outputs constrain the solar model, and in particular the observations of the luminosity and the radius are used to calibrate the models. A schematic view of the various regions characterizing the Sun from the interior to some of its outer features is shown in Figure 1. Davis and coworkers (Bahcall and Davis, 1976) were the first who tried to observe the neutrinos emitted from the solar core with a radiochemical detector (the latter was awarded a Nobel Prize in 2002). These early experiments showed a significant difference from the expected neutrino flux with only one third of the expected solar neutrinos actually measured. The reason for the missing neutrinos, the so-called "neutrino problem", was ascribed either to measurement uncertainties, or to an incorrect solar model or to an inappropriate knowledge of neutrino physics and of atomic cross-sections.

Concerning neutrino physics, already in the late fifties the idea of neutrino oscillations was introduced by Pontecorvo (1957) and later by Gribov and Pontecorvo (1969) who proposed that the discrepancy noticed could

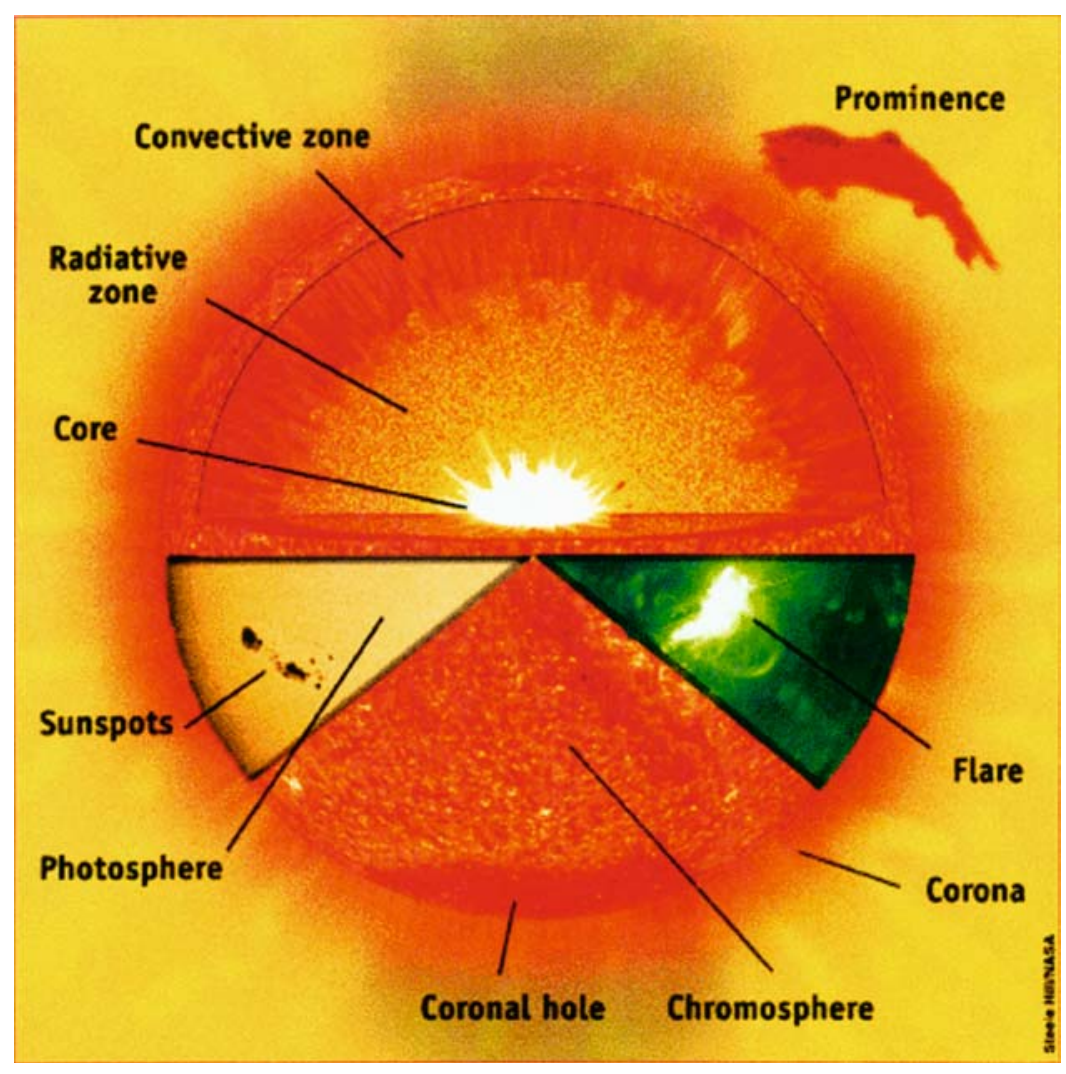

Figure 1. Schematic diagram of the Sun. Credit: SOHO, ESA/NASA. 
be due to an inadequacy of the particle physics model rather than in the standard solar model. They suggested that neutrinos oscillate between different states, or flavours, on their way from the Sun to the Earth. Wolfenstein (1978) and Mikheyev and Smirnov (1985) showed that the effects of matter on neutrinos moving through the Sun increased their probability for oscillation if their mass should fall in a certain range.

In parallel with neutrino detection, the technique of helioseismology provides a tool for the study of the solar interior. The discovery of sound waves (5-min oscillations) in the Sun (Leighton et al., 1962; Deubner, 1975), and their theoretical interpretation (Ulrich, 1970; Leibacher and Stein, 1971), led to an intense development of that technique, which is basically a Fourier analysis of solar acoustic waves. The sound waves can be observed via the Doppler shifting of light coming out of the solar surface, the photosphere, or via tiny variations in the spectral as well as in the total solar irradiance. They are easily accessed by ground based observation. The solar interior transmits sound very well, forming a spherical acoustical resonator. The noise generated by the convective processes near the surface is filtered due to interference, and only the resonant modes survive. The resulting oscillations are called acoustic or pressure waves (p-modes), and they are mostly non-radial.

The oscillation modes are trapped in spherical-shell cavities starting at the visible surface and extending inward. The periods of the waves depend on their propagation speeds and on the depths of their resonant cavities. Thus a large number of resonant modes within different cavities allow probing of the sound speed and density from just below the solar surface down to the core of the Sun.

As for any spherical oscillator, the angular component of the wave function is described by spherical harmonics, characterized by the degree and azimuthal-order quantum numbers, $l$ and $m$. The number of nodes of the oscillation pattern in the radial direction is denoted $n$. While the outer limits of all the cavities are confined to a narrow region just below the photosphere, the depth of a given cavity depends on the frequency and on the spherical harmonic degree $l$ of the associated mode. Besides the standing acoustic or p-modes, at relatively short wavelengths, surface-gravity waves (f-modes) can be observed. The largestscale modes of low frequency, the pure gravity modes (g-modes), penetrate deep into the solar interior, probing the innermost parts of the Sun. These latter have not yet been unambiguously detected in the Sun (cf., Section 7.1). The frequencies of the oscillation modes of the same $n$ and $l$ and different $m$ are degenerate for a spherically symmetric nonrotating system. Rotation lifts the degeneracy, and for solar p-modes the measurement of the rotational splitting can be used to deduce the rotation in the solar interior. 
Precision frequency measurements of the modes are thus the main goal of all helioseismology observations. Unfortunately, interruptions in the measurements, due to days and nights or bad weather, induce parasitic signals in the Fourier analysis. Three different possibilities were envisaged to handle that problem: (1) observations from the Earth's polar regions, (2) a network of observatories around the Earth, or (3) measurements with an uneclipsed instrument in space.

Using a long uninterrupted time series of solar observations of 4 days at the South Pole during the Antarctic summer, Grec et al. (1980) could dramatically increase the accuracy of the oscillation measurements, thus underlining the need for uninterrupted time-series and setting the stage for observational helioseismology. Figure 2 shows a power spectrum of their historical measurements.

The solar model could subsequently be improved down to roughly $10 \%$ from the core (Christensen-Dalsgaard and Gough, 1981; Stein, 1982; Deubner and Gough, 1984; Gough, 1985; Stix, 2002). Further improvement was achieved by comparing the velocities as deduced from the observations with those from models of the solar interior (Christensen-Dalsgaard et al., 1985; Gough, 1985). For the first time, the core of the Sun could be analysed and probed to an amount of detail never achieved before.

Observational results on solar internal rotation were obtained by Duvall et al. (1984). They found that much of the solar interior rotates slightly less rapidly than the surface. This result differed largely from what had been expected from stellar models, namely that the rotation rate would depend on the distance from the rotation axis. The first observations of the rotational splitting of the modes with respect to the azimuthal order $m$ also

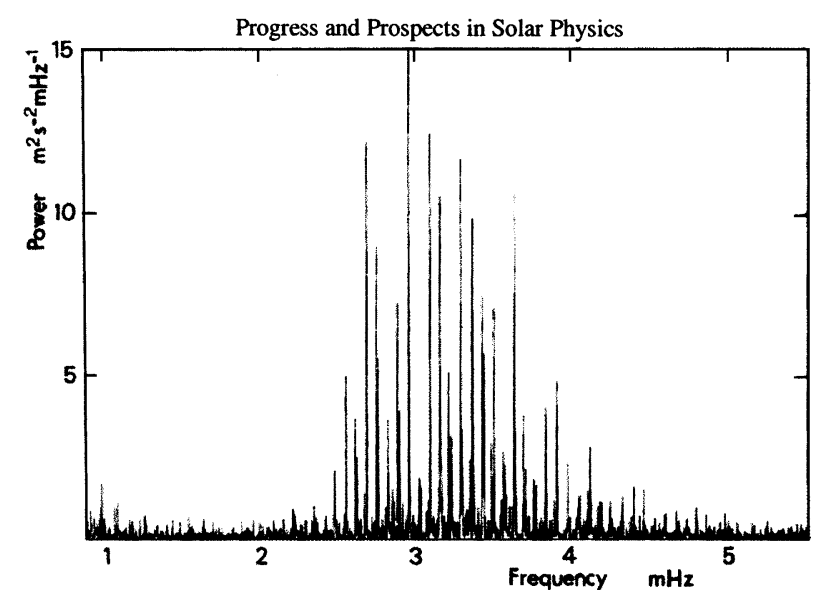

Figure 2. Power spectrum of the South Pole data recorded during the first week (6 days) of January 1980 (from Grec et al., 1983). 
constrained the latitudinal variation of rotation (Brown, 1985; Duvall et al., 1986). Their results indicated that the rotation varies from the surface down to the convection zone whereas the radiative zone rotates roughly uniformly (Brown and Morrow, 1987; Brown et al., 1989; Thompson, 1990). At the base of the convection zone where the transition between the latitudinal variation of rotation and uniform rotation takes place, layers of rotational shear have been discovered (Dziembowski et al., 1989; Goode et al., 1991; see also the review by Thompson et al., 2003). The narrow region of the transition between the two rotational regimes, the tachocline, is generally believed to be the location of the dynamo region, and of great importance for the generation of the large-scale solar magnetic field (Dziembowski et al., 1989).

\subsection{SOLAR MAGNETISM AND SOLAR VARIABILITY}

The Sun is variable on all time and on all spatial scales (Alexander and Acton, 2001; Solanki, 2002). The long time scales range from the solar (sunspot) cycle of 11 years (Schwabe, 1838) or the 22 years cycle of magnetic polarity inversion (Hale and Nicholson, 1925) to variations on even longer scales which manifest themselves in variations of the intensity and duration of the solar activity cycle.

Nearly all phenomena of solar activity are indications of the solar magnetic field. Hale (1908), using the Zeeman effect, identified sunspots as regions of strong magnetic fields, often appearing in pairs of opposite polarity (Hale et al., 1919). After the invention of the magnetograph (Babcock and Babcock, 1955), much weaker fields could be studied. The magnetic field is divergence-free, and thus the total magnetic flux through the solar surface is always zero. The eruption of flux through the surface is in form of loops. Outside sunspots the magnetic field is concentrated in thin flux tubes (Stenflo, 1973) with diameters of a few hundred kilometers. Groups of smaller magnetic elements form bright faculae and a network. The intersections of the largest flux tubes with the solar surface form the dark sunspots with a diameter of 4000-60,000 km. They consist of a darker umbra and a less dark penumbra; their field strength averaged over the whole spot is 0.1 to $0.15 \mathrm{~T}$ (1000 to $1500 \mathrm{G})$, with peak strengths around 0.2 to $0.35 \mathrm{~T}$ (2000 to $3500 \mathrm{G})$.

The manifold and interesting dynamics of sunspots was discovered early last century. Evershed (1909) observed a horizontal outflow in the photospheric layers of penumbrae along thin horizontal flux tubes. This outflow decreases with height and turns into an inflow at chromospheric heights. Biermann (1941) first proposed that the reduced brightness and temperature of the spots is due to the inhibition of convection by the magnetic field. The subsurface structure of sunspots is still not fully known. Parker 
$(1979 \mathrm{a}, \mathrm{b}, \mathrm{c})$ proposed a model in which sunspots are described as consisting of a single monolithic flux tube above the surface and of a crowd of small flux tubes somewhere below the surface (spaghetti or jellyfish model). Local helioseismology (cf., Section 5.2) can provide some constraints on the subsurface magnetic fields.

A comprehensive summary of the large field of sunspot research is given by Solanki (2003).

The sunspot number (see, e.g., Stix, 2002, for a definition) is the most widely used indicator for the solar cycle. Not only does the number of spots vary during the cycle but also their location. The first spots of each cycle appear at about $30^{\circ}$ to $35^{\circ}$ latitude in both hemispheres, and with the advancing cycle the zones of sunspot occurrence migrate towards lower latitudes. The last spots of each cycle appear normally within $\pm 10^{\circ}$ of the equator. This migration is often shown in so-called "butterfly" diagrams, an example of which is given in Figure 3.

The solar magnetic field is mainly a superposition of a toroidal part, i.e., a field whose lines of force are circles around the solar axis, and a poloidal part, with field lines in meridional planes. The most important property of the solar poloidal field is its cyclic reversal which takes place at the poles around the time of maximum sunspot activity. It was generally believed that a dynamo process in the convection zone was responsible for the magnetic activity and for the solar cycle. Several basic processes like
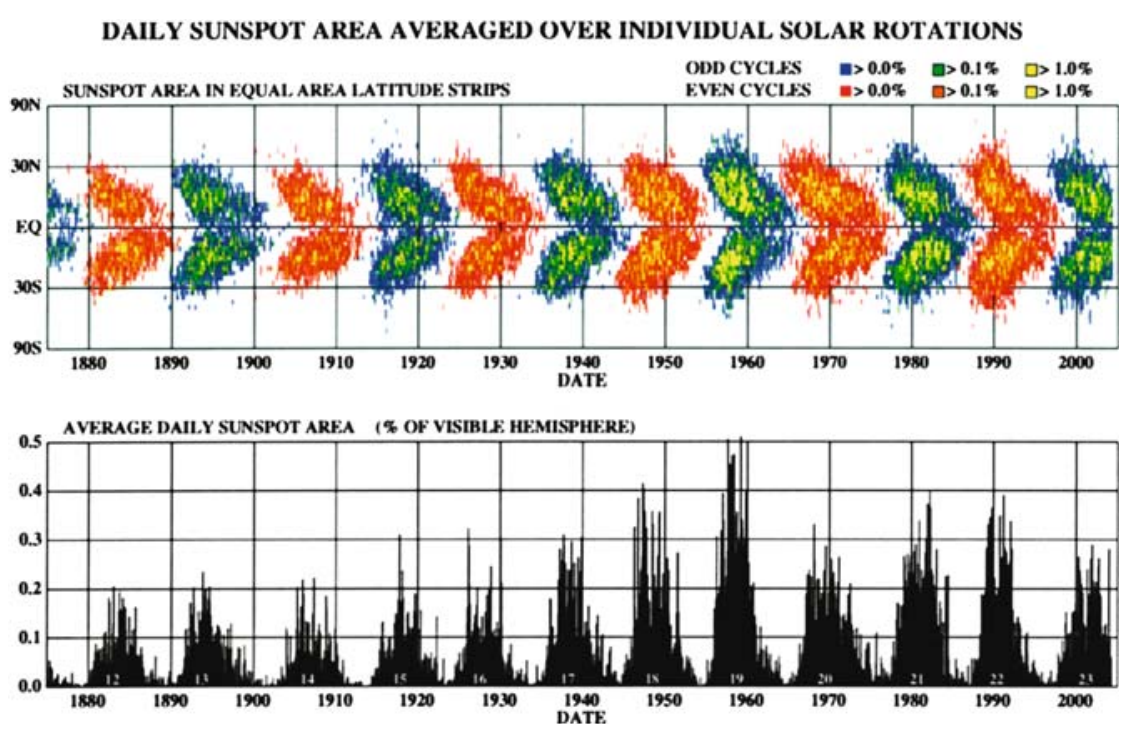

Figure 3. The so-called "butterfly" diagram, showing the location and area of the sunspots on the Sun during the cycles 12 to 23 . The lower part shows the fraction of the Sun covered by the spots. Image courtesy of D. Hathaway. 
differential rotation, buoyancy, and supergranulation were invoked also in order to distribute the fields into the observed patterns of magnetic concentrations (Babcock, 1961; Leighton, 1969).

Further up in the atmosphere, the magnetic pressure overcomes the gaseous pressure and the field plays a dominant role by confining the plasma and structuring the atmosphere. Indeed, the spatial distribution of coronal plasma has been shown to be directly related to the structure of the solar magnetic field, in particular magnetic loops have been observed during eclipses and from space. These loops, which consist of hot and dense material (trapped gas) that produces bright emission, have been the subject of several theoretical and observational studies (Landini and Montsignori Fossi, 1975; Craig et al., 1978; Foukal, 1978; Rosner et al., 1978; Jordan, 1980; Priest, 1981; Withbroe, 1981); the latter, however, were limited to rather modest (a few arcseconds) spatial resolution and also to snapshots lasting the time of a rocket flight.

Flares, the explosive releases of a large portion of energy in sudden brightenings of the active regions, had been observed in $\mathrm{H} \alpha$ and also in white light (cf., Section 6.1, see also the discussion in the later paragraphs). The flaring process is associated with the interaction of magnetic loops of opposite polarity (Giovanelli, 1947; Dungey, 1958; Gold and Hoyle, 1960) and assigned to magnetic reconnection (Petschek, 1964). Radio emission from solar flares (cf., Section 2.3.1) is due to gyrosynchrotron radiation from electrons of energy larger than $1 \mathrm{MeV}$.

Another important problem related to the activity cycle was the stability of the total radiative output of the Sun, the so-called solar constant. It was unclear whether the radiation integrated over all wavelengths was indeed an invariant. Unfortunately, due to the perturbations of the Earth's atmosphere, ground-based measurements did not have sufficient precision to detect possible variations. The early measurements of the total solar irradiance (TSI) were indeed consistent with a constant solar radiative output whose observed variability could be caused by the Earth's atmosphere (Smith and Gottlieb, 1974). However, the early results obtained from space hinted at a dependence on the solar magnetic cycle as well, and a discussion of this topic led to the inclusion of the Active Cavity Radiometer Irradiance Monitor (ACRIM) on the Solar Maximum Mission (SMM) (White, 1976, and articles in these proceedings; Foukal et al., 1977) and subsequently of similar devices on other missions, in particular on SOHO (see Section 4.3).

\subsection{THE SOLAR ATMOSPHERE}

The temperature inside the Sun decreases from the core to the outer parts, the photosphere. A continuous decrease of temperature from the photo- 
sphere into the outer solar atmosphere would then be thermodynamically consistent. However, the temperature rises abruptly in the chromosphere from a minimum of $4300 \mathrm{~K}$ to several $10^{4} \mathrm{~K}$ and within a few hundred kilometers to values of several $10^{6} \mathrm{~K}$ in the corona. Where one would expect a cool non-emitting outer sheath we find a hot, luminous and extensive outer envelope. This somewhat unexpected stratification of the solar atmosphere into these different temperature layers was first observed with early space instrumentation (Bonnet, 1968). These observations were of modest resolution and their rather simplistic interpretation assumed a plane-parallel atmosphere (Figure 4). The first successful models of the solar atmosphere were based on that assumption (Vernazza et al., 1973, 1981; Fontenla et al. 1993). However, due to the rapid decrease of the density with height, the upper atmosphere is highly structured and sensitive to all manifestations of solar activity. In fact, today, the concept of a quiet atmosphere is indeed questionable.

\subsubsection{Early space and radio observations}

The first successful solar observations from space were performed by the US Naval Research Laboratory in 1946, when an UV spectrometer was launched and recovered on board a V-2 rocket (Tousey, 1953). Around 1960, a short-wavelength spectrum was available (Hinteregger, 1965; or the review by de Jager, 2002), which clearly confirmed a change in the spectrum from absorption to emission at about $150 \mathrm{~nm}$, and thus a continuous

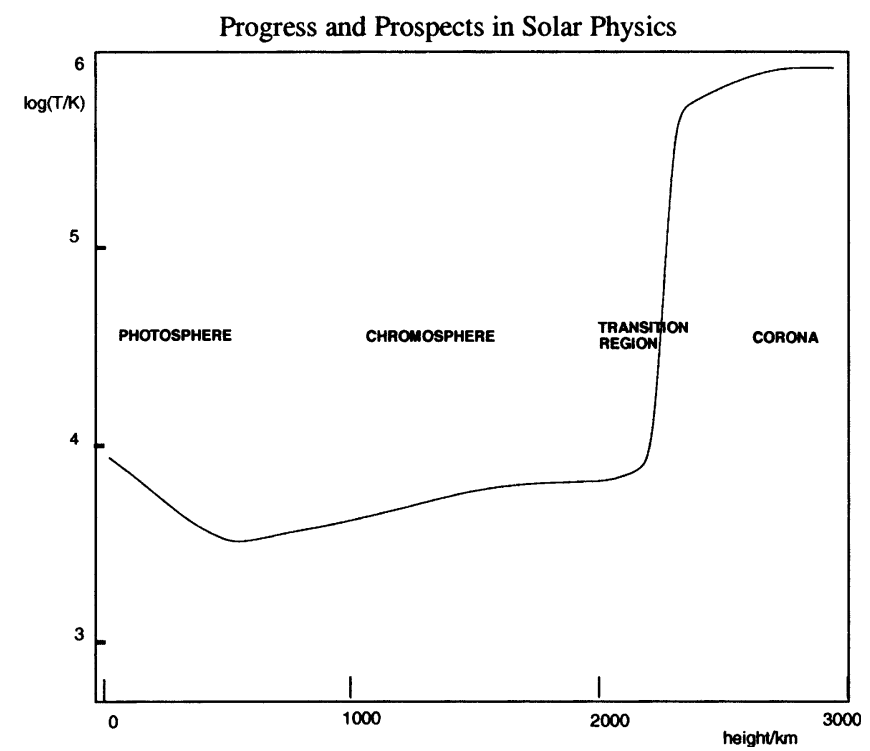

Figure 4. Temperature structure of the solar atmosphere as derived from early EUV observations (after Vemazza et al., 1973). 
temperature increase outward of the region where the $150 \mathrm{~nm}$ radiation is emitted. Solar X-rays were first detected in 1948 (Burnight, 1949) and proved to be highly variable. The first hard X-and $\gamma$-rays associated with a flare were observed in March 1958 during a balloon flight (Peterson and Winckler, 1959). A summarizing overview of the solar emission spectrum ranging from 10 to $100 \mathrm{keV}$ was given by Elwert (1961).

Soon it was realized that monitoring with rockets was not appropriate to capture these variable events, but that satellites were necessary to observe the flaring activity in the high-energy range. Around 1960, the satellite era began with the Soviet Sputniks carrying photon counters for measuring radiation between 0.1 and $12 \mathrm{~nm}$ and the hydrogen resonance line Lyman $\alpha$. In March 1962 NASA started the Orbiting Solar Observatory (OSO) series allowing quiet Sun as well as transient events to be studied through high-resolution EUV and X-ray spectra (Jordan, 1972; Walker, 1972).

The Earth's atmosphere is transparent to radio frequencies; thus, measurements in the radio wavelength range ( $\mathrm{mm}$ - to m-waves) can be made with ground-based antennas. Radiation from the Sun in the radio wavelength band is emitted in the upper atmosphere and in the corona. It was first detected by Southworth in the US and Hey in Britain in 1942 (Hey et al., 1948). This radiation had been predicted earlier, but mainly due to the poor sensitivity of the instruments detection was not possible. With the use of better receivers, the study of solar millimeter wave radiation started around 1970. For reviews on solar and stellar radio observation see, for example, Dulk (1985) and Güdel (2002), and for solar transient events Bastian et al. (1998) and Kundu and White (2003).

Particles accelerated in the solar atmosphere generate various kinds of radio emission, and the solar radio spectrum consists of a superposition of the signatures of many different processes. Besides a thermal quiet Sun spectrum, there is a slowly varying component that correlates well with the solar activity cycle, and the radio flux at $10.7 \mathrm{~cm}$ is often used as a proxy for solar activity (Stix, 2002). At longer wavelengths, the radio spectrum becomes dominated by radio bursts, many of which are associated with flares. These bursts have been classified as types I to V.

Possible radio emission mechanisms at $\mathrm{cm}$ - and mm-waves are gyrosynchrotron radiation by mildly relativistic electrons, synchrotron radiation by highly relativistic electrons, and thermal bremsstrahlung by electron-ion collisions in the plasma. Of particular interest in the context of flaring processes are the type III radio bursts. They are radio emissions generated by streams of energetic electrons that are injected from the Sun into interplanetary space and often associated with some explosive events. These radio bursts were among the first types of meter-wave bursts discovered in the 1940 s, and the drift of the emission to lower frequencies with time was 
interpreted by Wild (1950) as the signature of a particle beam propagating upward through the corona at a speed of 0.2 to $0.6 \mathrm{c}$. Thus, these suprathermal electrons reach the orbit of the Earth in about 20 to $40 \mathrm{~min}$, following the interplanetary extension of the solar magnetic field, the change in frequency being due to the change of the density of the plasma that the particles excite on their way. Consequently, the type III radio bursts can be used as a diagnostic of the acceleration process of electrons in flares, as tracers of the magnetic field lines along which the beams propagate, and of the ambient density along their trajectory.

\subsubsection{The photosphere}

To simplify, the photosphere is the visible surface of the Sun. It extends from a region of temperature $6000 \mathrm{~K}$ about $400-500 \mathrm{~km}$ upward to the atmospheric temperature minimum of $4300 \mathrm{~K}$, which is roughly the base of the chromosphere (Figure 4).

With the use of the first telescopes, the various components and features of the photosphere had been described, such as the dark sunspots and the bright faculae, which are associated with magnetic activity. The photospheric granulation exhibits a "mottled" structure of brighter areas, typically $1000 \mathrm{~km}$ in diameter, surrounded by a darker network. Detailed studies of the granulation have been made with balloon-borne telescopes (Danielson, 1961; Mehltretter, 1978), aboard Spacelab 2 (Title et al., 1989) and from the ground. The granulation is the surface signature of overshooting convection and the bright granules identify the hot upflowing gas from the convection zone whereas the darker lanes mark the cool downflowing gas. An excellent review on solar convection in given by Spruit et al. (1990). On larger scales, a structure of 5 to $7 \times 10^{6} \mathrm{~m}$ called mesogranulation (November et al., 1981), and of 20 to $30 \times 10^{6} \mathrm{~m}$ called supergranulation (Simon and Leighton, 1964) had been found. Giant convection cells, reaching deep into the interior of the convection zone, had been predicted by Simon and Weiss (1968). Several attempts to detect these cells had been made, but only very few gave hints on their existence (Cram et al., 1983; Snodgrass and Howard, 1984). Brown and Gilman (1984) gave an upper limit of their velocity signal $(10 \mathrm{~m} / \mathrm{s})$ and showed that their detection required very high precision.

The photospheric composition was studied since the early days of spectroscopy, the middle of the 19th century, when Joseph von Fraunhofer (1787-1826) first used the dark lines of the solar spectrum (later termed Fraunhofer lines) as reference points for the measurement of refraction indices. Later, Kirchhoff and Bunsen (1860) established the theory of spectral analysis of chemical elements, investigated the Fraunhofer lines, and paved the way for spectroscopy. Solar elemental abundances were determined by Russell (1929) and Goldberg et al. (1960); see also Grevesse and 
Sauval (1998). Similarly, typical phenomena of the photosphere like limb darkening and the Fraunhofer absorption spectrum have been used to determine the temperature stratification (Bonnet, 1968).

\subsubsection{The chromosphere and transition region}

The chromosphere has been observed extensively from the ground at the beginning and at the end of totality of solar eclipses. Alternatively, it can be observed in the cores of strong absorption lines at visible or near-ultraviolet wavelengths (e.g., the $\mathrm{H} \alpha \mathrm{Ca}$ II $\mathrm{H}$ and $\mathrm{K}$ lines at around $390 \mathrm{~nm}$ and the $\mathrm{Mg}$ II $\mathrm{h}$ and $\mathrm{k}$ lines at around $280 \mathrm{~nm}$ ). Because of its high temperature the chromosphere can be extensively studied through the UV, EUV and $\mathrm{X}$-ray part of the spectrum. Observations of the emission lines emitted in these ranges allow the determination of temperature and density structure of the hotter atmosphere (Pottasch, 1963, 1964; Gabriel and Jordan, 1969; Héroux et al., 1972).

The chromosphere is the lowest part of the solar atmosphere where the temperature increases outwards and which thus requires energy input. Biermann (1946) suggested that acoustic waves generated in the turbulent flow field of the upper convection zone would dissipate in the chromosphere and be the source of the heating. The chromospheric network consisting of lanes of enhanced brightness along the boundaries of the supergranulation cells (Bonnet et al., 1982) is a common feature of most spectral lines formed at chromospheric temperatures. Skumanich et al. (1975) showed that the chromospheric emission directly overlying the photospheric magnetic field elements is enhanced.

Magnetograms obtained near the solar limb in chromospheric lines showed patches of almost horizontal field, which were interpreted as the base of a magnetic canopy (Giovanelli and Jones, 1982), where the plasma changes its character from being dominated by the gas pressure to being dominated by the magnetic pressure and the field lines spread out horizontally.

The transition region is generally regarded as the thin layer of the thermocline between the dense and cold chromosphere and the hot and tenuous corona. Early observations of the intensities of EUV emission lines (Pottasch, 1963; Athay, 1966; Dupree and Goldberg, 1967; Dupree, 1972) hinted at a thin stratified layer in the simplistic assumption of a plane-parallel atmosphere. Measurements of spectra obtained by the Orbiting Solar Observatories and Skylab (Vernazza et al., 1973; Rosner et al., 1978; Bonnet et al., 1980; Vernazza et al., 1981; Fontenla et al., 1993), however, contributed to reveal the inadequacy of this assumption to represent the chromosphere's properties.

Along the solar limb, several types of inhomogeneities were found at chromospheric and transition region temperatures, e.g., prominences 
(Sheeley et al., 1975), corresponding to clouds of cooler material embedded in the hot corona and suspended against gravity by the magnetic field. Skylab observed spicules and macrospicules (Zirin, 1966), that are formed by columns of gas out of the solar limb, and that had already been noticed much earlier by Secci (1870). Images taken from rockets and Spacelab, such as with the Transition Region Camera (TRC) in the hydrogen Ly- $\alpha$ line revealed loops at the $1^{\prime \prime}$-level (corresponding to roughly $700 \mathrm{~km}$ on the Sun) in the network boundaries (Bonnet et al., 1980), and showed the existence of a very complex pattern of dynamic phenomena in the transition region. Velocities of up to $500 \mathrm{~km} / \mathrm{s}$, far in excess of the local sound speed, were observed by Brueckner and Bartoe (1978). Dere et al. (1987) also used spatially resolved spectra from the High Resolution Telescope and Spectrograph (HRTS) to provide evidence of the complex fine structure of the transition region.

\subsubsection{The corona}

The invention of the coronagraph by Lyot (1939) led to the detection of a number of emission lines in the corona beyond the brief moments of solar eclipses. The newly discovered lines indicated temperatures of at least $10^{6} \mathrm{~K}$. A historical review of the corona and the solar wind concept is given by Parker (2001).

An outstanding question has been the interpretation of this high temperature and of the heating processes that sustain such a hot corona. Withbroe and Noyes (1977) estimated the necessary heat input to be approximately $3 \times 10^{21} \mathrm{~W}$. It was supposed rather early that some kind of mechanical energy, like convective overturning below the visible surface of the Sun, might provide mechanical work on the plasma and magnetic field. This in turn would generate acoustic waves, internal gravity waves, Alfvén waves, or other magnetohydrodynamic (MHD) wave modes. These waves would propagate into the chromosphere and the corona where they would be dissipated by viscosity, Ohmic diffusion and thermal conductivity and thus convert their energy into heat (Alfvén, 1947; Schwarzschild, 1948; Biermann, 1948; Schatzmann, 1949). Later, it became clear that acoustic waves cannot transport enough energy beyond the chromosphere as was shown using results from OSO 8 (Athay and White, 1978; Bruner, 1981) and ground-based observations (Schmieder and Mein, 1980). Parker (1983, 1988) suggested that the energy necessary for coronal heating could be dissipated in flare type events generated by changes in the magnetic field topology or by the collision of field lines (Sweet, 1969; Švestka, 1976). However, the exact processes involved in the heating process were not known.

Through eclipse as well as radio and EUV and X-ray observations, like all other parts of the solar atmosphere, the corona was found to be made up of a multitude of different structures. 
Coronal holes (Waldmeier, 1957, 1975), i.e., regions that appear dark in coronal emission lines, were known very early to overlay open unipolar magnetic field regions Poletto et al., 1975; Timothy et al., 1975; Bohlin and Sheeley, 1978; Zirker, 1977) and recognized as the sources of the highspeed solar wind streams (Krieger et al., 1973; Nolte et al., 1976; Bohlin, 1977). Active regions are bright in coronal emission lines and consist of complex systems of magnetic loops with larger magnetic field intensity, temperature and pressure. The corona outside holes and active regions is called the quiet corona and composed of loops of lower temperature and pressure than in active regions. Large magnetic loop structures were known to be present in the solar outer atmosphere from ground-based observations, e.g., loop prominences observed at the limb, or from observations of few rare coronal lines falling into the visible spectrum. However, only after the availability of X-ray observations, using rockets and satellite instruments, in particular Skylab, were these magnetic loops identified as the source of the entire X-ray emission (Vaiana et al., 1973; Vaiana and Rosner, 1978). Observations in the EUV wavelength range revealed cool material to be present within the coronal loop structures (Raymond and Foukal, 1982). Although several observational and theoretical studies were concerned with the stability and equilibrium properties of coronal loops (Foukal, 1975; Antiochos, 1979; Kuin and Martens, 1982), many of the phenomena associated with these loops needed more and better data to be understood. Also, little was known about the time variation of the loop geometry, their interaction with the rest of the coronal in particular their reconnection. It was supposed that the magnetic field, instead of passively shaping the loops, could in fact play an active role in providing the mechanism for transporting and dissipating the energy needed to maintain the corona.

\subsubsection{The fast and slow solar wind}

It has been known for a long time that charged particles emitted by the Sun interact with the Earth's magnetic field and cause the aurorae. The observation that the plasma tails of comets always point radially away from the Sun led Biermann (1951) to postulate that there exists a solar corpuscular radiation which is continuous rather than intermittent. Parker (1958) calculated that the inward pressure of the interstellar medium was too weak for the solar atmosphere to be in hydrostatic equilibrium and introduced the term "solar wind" to describe the outward flowing part of the solar corona supplying the pressure to balance the pressure exerted by the local interstellar medium. Moreover, he found that the interplanetary extension of the solar magnetic field would be drawn out into Archimedian spirals due to the rotation of the Sun. 
Most of Parker's theoretical predictions were confirmed by the first interplanetary probes, like the Soviet Lunik 2, launched in September 1959, which measured a stream of high-speed ions from the Sun (Gringauz et al., 1964), and the US spacecraft Mariner 2 which also revealed the existence of a two-velocity regime of the solar wind (a slow wind of a velocity of around $350 \mathrm{~km} / \mathrm{s}$, and a fast wind of around $750 \mathrm{~km} / \mathrm{s}$ ), as well as the sector structure of the interplanetary magnetic field (Neugebauer and Snyder, 1966). The existence of two kinds of solar wind flow was also revealed by the Helios 1 and 2 spacecraft, launched in 1974 and 1976, respectively (Rosenbauer et al., 1977). Using solar wind measurements on board IMP-7 (one of a series of Interplanetary Monitoring Platforms) Hovestadt (1974) found an enhancement of the chemical elements of the corona with low first ionization potential (FIP $\leq 10 \mathrm{eV}$ ) relative to those with higher first ionization potential in comparison to their photospheric abundance ratio. This enhancement was later termed the "FIP-effect". For a historic overview see Parker (2001) and Neugebauer and von Steiger (2001). The source of the wind and its acceleration mechanism(s) were, however, unexplained.

Rocket experiments reported blue shifts of emission lines formed in the transition region and in the corona in a coronal hole (Rottman et al., 1982). Thus it was concluded that an emergent flux of matter, the solar wind, could already been measured low in the transition region and in the inner corona. However, the angular resolution of the measurements was not sufficient to discriminate exactly between open and closed field structures.

\subsubsection{Coronal mass ejections}

Another phenomenon that was observed in the corona were transient events, causing large scale disturbances such as shock waves in the solar wind (Sheeley et al., 1985). Already Chapman and Ferraro (1931) had suspected that solar flares would eject streams of plasma into interplanetary space that would disturb the geomagnetic field several days later. Such events were indeed observed by the coronagraph on Skylab (MacQueen et al., 1974). Shortly after their discovery these events were called "coronal transient disturbances" or "temporary coronal depletions" (Gosling, 1974). Only later were they called "coronal mass ejections" or CMEs. Many observations of these events were made with the Solar Maximum Mission (SMM), launched in 1980, suggesting that isolated magnetic loop structures or magnetic clouds were associated with the ejected plasma and the interplanetary shock wave (Burlaga et al, 1981). It was thereby suspected that changes of magnetic topology and magnetic reconnection could play a major role in the initiation of these CMEs, themselves the cause of large scale interplanetary and magnetic disturbances, in particular in the Earth's magnetosphere. 


\section{The progress in ground-based observations}

Numerous high-standard solar observatories are presently operated all over the world. For a description and a list of the facilities we refer to Stix (2002) and the article by Fleck and Keller (2003).

Ground-based astronomical observations are certainly cheaper than those obtained from space. They have nevertheless always been compromised by cloudy weather or turbulent conditions in the Earth's atmosphere disturbing the seeing along the light path, limiting the angular resolution to $1^{\prime \prime}$ on average with few noticeable exceptions at the best sites. Thus, the largest solar telescopes had diameters of only 1-1.5 m, since going larger did not enhance resolution. The seeing problem, however, has recently been overcome by new correction techniques leading to spectacular improvements in the spectral bands that are capable of penetrating the Earth's atmosphere.

\subsection{SOLAR TELESCOPES AND ADAPTIVE OPTICS}

Adaptive optics (AO) refers to optical systems that actively compensate for optical effects introduced by the atmosphere between the object and its image. Recently, several Earth-bound solar observatories have installed adaptive optics systems on their telescopes. One of the first was operated at the Dunn telescope of the National Solar Observatory (NSO) at Sacramento Peak (Rimmele et al., 2003; Ren et al., 2003). It has been the first to achieve resolutions of about 0.2". In May 2002, the Swedish 1-m Solar Telescope (SST) at La Palma started operating and obtained solar images with an unprecedented spatial resolution of roughly $0.13^{\prime \prime}$ (90 km on the Sun) showing spectacular structures like the sunspot penumbra shown in Figure 5 (Scharmer et al., 2002).

Other facilities equipped with advanced AO systems are, e.g., the German Vacuum Tower Telescope (VTT) in Tenerife, or the McMath-Pierce Solar Telescope at Kitt Peak (currently the world's largest solar telescope with an aperture of $1.5 \mathrm{~m}$ ). Many more such facilities exist worldwide, and a 4-m aperture telescope, the Advanced Technology Solar Telescope (ATST http:// atst.nso.edu), is planned to be built around 2006 and to be commissioned in 2010. It will also operate in the infrared and will be able to measure polarization. Employing the most recent advances in adaptive optics, resolutions of better than $0.1^{\prime \prime}$ will be achieved.

\subsection{THE SEARCH FOR CONTINUOUS 24-HOURS COVERAGE: OBSERVATION NETWORKS FOR HELIOSEISMOLOGY}

Uninterrupted observations from a single Earth-based site are not possible, and all locations, apart from within the Arctic and Antarctic circles, have 


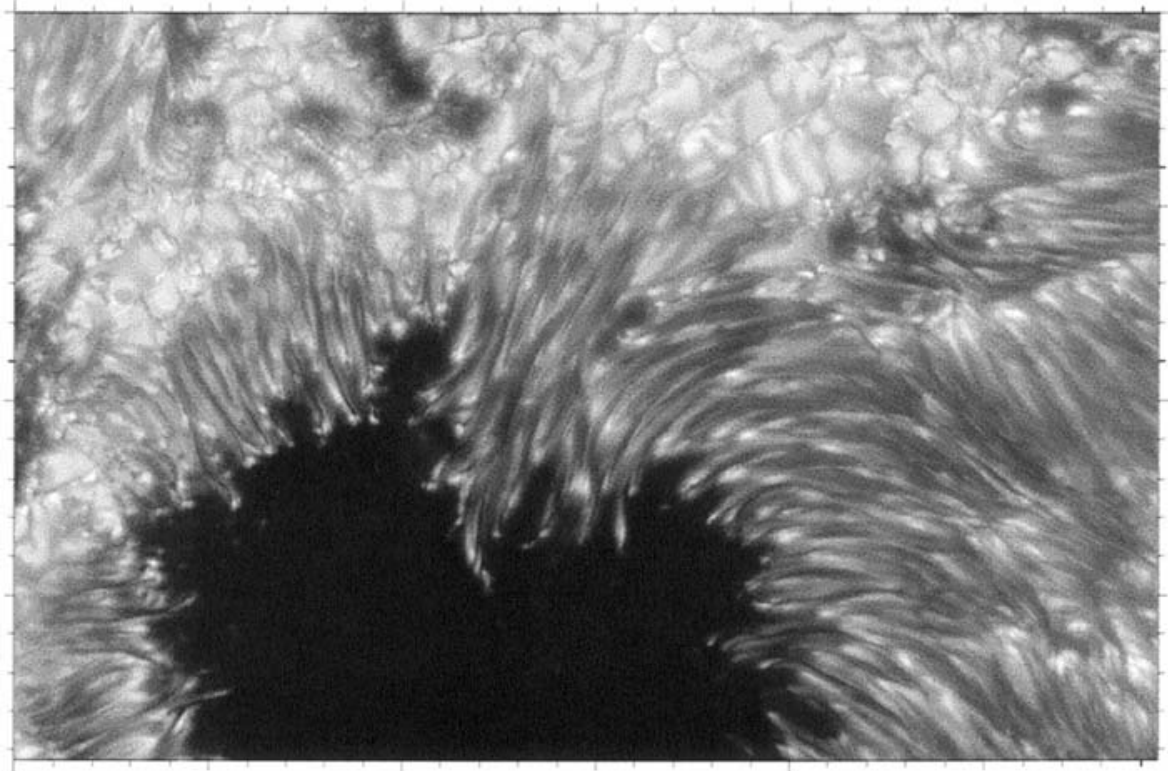

Figure 5. Part of a large sunspot recorded on 15 July 2002 with the Swedish 1-m Solar Telescope at La Palma. This image was post-processed using the so-called Phase-Diversity Technique (Löfdahl and Scharmer, 2003), making it the highest-resolution solar image ever. The distance between two tickmarks is $1000 \mathrm{~km}$. Credit: Royal Swedish Academy of Sciences.

day-night variations which convolve into the spectrum of the solar oscillations. Continuous observation requires a network of instruments distributed around the Earth, i.e., at different longitudes. Such networks have to include a sufficient number of stations at well-chosen locations, in order to provide redundancy in case of instrument failures and bad weather conditions.

Networks do provide an alternative to space measurements. Besides being much less expensive than space systems like, e.g., SOHO (see Section 4.3), the maintenance and data access of their instruments are much easier since components can be updated or exchanged on request.

Five such networks have been developed since the 1980s:

The Birmingham Solar Oscillations Network (BiSON) started in 1981 to monitor low-degree solar oscillation modes with a network of six remote solar observatories (Chaplin et al., 1996).

IRIS (International Research on the Interior of the Sun), a network of six stations (Fossat, 1991) observes since 1989 the full disk line-of-sight velocity.

The Taiwan Oscillation Network (TON), operating since 1993, takes full-disk solar images of high resolution currently using four telescopes (Sun et al., 2003). 
The Global Oscillation Network Group (GONG) (Harvey et al., 1996) is using six stations in operation since 1995 with extremely sensitive and stable velocity imagers.

The ECHO-LOWL network is operated by the High Altitude Observatory, of the National Center for Atmospheric Research in Boulder, Colorado. The first ECHO instrument, at the Observatorio del Teide, Tenerife, Spain, has been operating since the end of 1999. In 2000, a second instrument was added on Mauna Loa. This latter instrument (LOWL) was originally designed for the simultaneous observation of low- and intermediatedegree solar oscillations with spatial resolution. It had been in operation from 1994 to 2000 on Mauna Loa, Hawaii (Tomczyk et al., 1995).

\subsection{NeUtRINO DETECTORS}

Neutrinos barely interact with matter, and the Sun is nearly transparent to them. This makes their detection particularly difficult. Luckily the solar neutrino flux is intense, and measurements have provided unique information about the physical conditions existing in the solar core. Some nuclei, e.g., the ${ }^{37} \mathrm{Cl}$ and ${ }^{71} \mathrm{Ga}$ isotopes, have a sufficiently large cross section to capture a fraction of the neutrinos (the yield is roughly one neutrino per $10^{20}$ ), and these isotopes are used in radiochemical detectors. Another method uses neutrino-electron scattering in water reservoirs, the scattered electron emitting Čerenkov light if its velocity exceeds the velocity of light in water. A description of the most important reaction chains, their energy and neutrino balances, and the various detector energy thresholds is given by Stix (2002). The first neutrino detector, a ${ }^{37} \mathrm{Cl}$ experiment, was built in the Homestake Gold Mine in South Dakota, USA (Davis, 1964). Examples of ${ }^{71} \mathrm{Ga}$-detectors are the Russian-US SAGE experiment in the Baksan underground laboratory in the Caucasus, or the GALLEX French-German-Israel-Italian collaboration in the Gran Sasso underground laboratory in Italy. Cerenkov water detectors have been built in the Mozumi mine near Kamioka (Kamiokande in 1987 and Super-Kamiokande in 1996). In 1998 Super-Kamiokande measured signals of oscillations of neutrinos generated in the Earth's atmosphere by interaction with cosmic rays (Fukuda et al., 1998).

The Sudbury Neutrino Observatory (SNO) is a Cerenkov detector using heavy water instead of ordinary water. It is situated in a nickel mine in Sudbury, Ontario, Canada, and is able to measure the ratio of the electron neutrino flux to the total neutrino flux (all flavours). In 2001 and 2002 the SNO group published the detection of non-electron type neutrinos (SNO, 2001, 2002) and confirmed the neutrino oscillations. These results imply that the total neutrino count is in excellent agreement with the standard solar model and the results of helioseismology, putting the solar neutrino enigma to rest. 


\section{The space revolution}

Following the first wave of pioneering space experiments, during the seventies and eighties of the last century, it became clear that in order to understand the mass and energy balance and the structure and dynamics of the solar atmosphere, observations with higher resolution and longer time series were indispensable. Because of the need to cover the crucial spectral ranges in the UV and X-ray in particular, these observations had to be conducted from space.

Most instruments then still suffered from severe difficulties in calibration and monitoring of their stability (Smith and Huber, 2002). Immense progress, however, was achieved when several elaborate and complementary well-calibrated "high-end" instruments were launched on satellites, most of them in the framework of international cooperation. Their originality derives from major progress in technologies, advanced computing and unique observing sites and orbits. Some of the most prominent ones are described in the following paragraphs.

\subsection{ULYSSES: THE FIRST OUT-OF-ECLIPTIC MISSION}

Originally, the out-of-ecliptic mission was a joint undertaking between ESA and NASA. Both agencies had the responsibility for one spacecraft each. NASA's satellite carried all imaging instruments. However, in 1981, NASA decided to abandon the project. ESA continued to work with NASA on the European satellite now called Ulysses that was launched by the US Space Shuttle in October 1990. After a close fly-by of Jupiter in February 1992, it entered a 6-year eccentric orbit around the Sun.

Ulysses carries a number of instruments for in situ studies of the solar wind and the interplanetary magnetic field, of interplanetary dust grains, particle composition and energies, plasma and radio waves. In addition, remote sensing instruments observe solar X-rays and cosmic rays. For a description of the mission see, e.g., the special issue of Astron. Astrophys. Suppl. Ser., Vol. 92 (1992). Ulysses has conducted the first-ever survey of the Sun's environment in space from the equator to the poles, i.e., from a heliocentric orbit inclined at $80^{\circ}$ to the ecliptic plane, over more than a solar cycle, covering a wide range of solar activity conditions. Ulysses is still in operation and foreseen to be active until 2008.

Thanks to its unique orbit, Ulysses has provided the first survey of the high-latitude heliosphere within $5 \mathrm{AU}$ (1 AU is the Sun-Earth distance, i.e., $1.5 \times 10^{8} \mathrm{~km}$ ) of the Sun over the full range of solar activity conditions. Key results to date include the first detailed measurements of the solar wind (see Section 2.3.5) from the Sun's polar regions, both at solar minimum and solar maximum, showing that the slow wind originates from low solar lati- 
tudes around the equator, while the fast wind originates mostly from the poles where extended coronal holes exist (Phillips et al., 1995); the discovery that the magnetic flux leaving the Sun and normalized to $1 \mathrm{AU}$ is the same at all latitudes (Smith and Balogh, 1995; Forsyth et al., 1996); the discovery that cosmic rays are not more abundant above the poles than near the ecliptic, probably because of the presence of Alfven waves that prevent them from penetrating the polar regions (Simpson et al., 1995; Heber et al., 1996); the discovery of energetic charged particle "reservoirs" surrounding the Sun (Roelof et al., 1992); the discovery of interstellar dust in the solar system (Grün et al., 1993), and the first direct measurements of interstellar helium atoms in the solar system (Banaszkiewicz et al., 1996).

\subsection{YOHKOH: THE STATE-OF-THE-ART X-RAY INSTRUMENT OF THE 1990S}

Yohkoh (Japanese for "sunbeam") was launched in August 1991 with substantial US and UK collaboration. It was designed for the study of solar $\mathrm{X}$-and $\gamma$-rays and optimally suited for the study of flares and transient phenomena in the corona. The satellite carried four instruments covering an energy range from soft X-rays to $\gamma$-rays up to $100 \mathrm{MeV}$. Yohkoh stopped functioning in December 2001. The mission is described in a sequence of articles in Solar Physics, Vol. 136 (1991). Yohkoh has monitored the X-ray corona over a full solar cycle and has revealed the detailed structure and dynamic nature of the corona and its evolution over nearly a complete solar cycle, in particular the loop systems (Tsuneta et al., 1992). It has also revealed the commonality of magnetic reconnection processes in the low corona and in active regions (Shimizu et al., 1992; Shibata et al., 1993), and contributed extensively to the study of solar activity and, in particular, of solar flares, CMEs, and X-ray bright points (cf., Sections 2.2 and 2.3.4), making it possible for the first time to obtain detailed observations of the onset of these phenomena and their clear relationships to coronal structures and magnetic fields (Masuda et al., 1994; Sakao et al., 1994; Hanaoka, 1996; Tsuneta, 1997).

\subsection{SOHO: THE MOST COMPREHENSIVE SET OF INSTRUMENTS TO STUDY THE SUN FROM THE CORE TO THE CORONA}

The Solar and Heliospheric Observatory (SOHO) is a joint venture between ESA (responsible for the spacecraft development) and NASA (responsible for its launch and operation in orbit). Both agencies were in charge of developing the scientific payload for European and American investigators. It was launched in December 1995 into a halo orbit around the first Lagrangian point $L_{1}$. This position, at $1.5 \times 10^{6} \mathrm{~km}$ sunward from Earth, permits an 
uninterrupted view of the Sun, which is especially useful for measurements requiring long time series, as, for example, the global oscillation studies.

Five instruments on SOHO take images and spectra in several wavelength bands from the ultraviolet to soft X-rays. The corona is observed out to a distance of approximately $32 R_{\odot}\left(1 R_{\odot}=696,000 \mathrm{~km}\right)$ with a set of three coronagraphs. Three instruments perform in situ measurements of the solar wind, especially of the energy distribution of its constituents. Another suite of three instruments is dedicated to helioseismology.

In the summer of 1998, SOHO accidentially lost its pointing attitude. As a consequence, it lost the inner coronagraph and its three gyroscopes. Special software was developed which operates very successfully and makes SOHO the first solar spacecraft that is operated without a gyro stabilization system. The SOHO mission is described by Fleck et al. (1995). Most results obtained with SOHO and its unique contribution to solar physics are extensively described in Section 5. These include, in particular, the first in depth measurement of solar internal rotation down to a few percent of the solar radius, observations of subsurface zonal shear flows and of temporal variations of the rotation near the base of the convection zone, showing the complex dynamics at the presumed location of the dynamo, major contributions to the corona heating problem, in particular the role of the magnetic field, the source and acceleration mechanisms of the fast and slow solar wind, continuous and extensive observations of CMEs and active phenomena on the disk and even on the "hidden face" prior to their appearance on the "visible face". The mission is planned to continue operations until 2007.

\subsection{TRACE: THE HIGHEST ANGULAR RESOLUTION ACHIEVED FOR UV IMAGING}

The Transition Region and Coronal Explorer (TRACE) is a NASA mission to image the solar corona and transition region at very high angular and temporal resolution. TRACE was launched in April 1998, and it carries a normal incidence telescope operating between 28 and $250 \mathrm{~nm}$. It is an advanced version of the EIT (Extreme-ultraviolet Imaging Telescope) instrument on $\mathrm{SOHO}$, and images fields of $8.5^{\prime} \times 8.5^{\prime}$ on the disk. The spatial resolution is below $1 \mathrm{~mol}$ I. Since the orbit is Sun-synchronous, long uninterrupted observations of the Sun are possible. Handy et al. (1999) describe the mission, which is foreseen to continue through 2010. Thanks to its unprecedented angular resolution, TRACE has revealed the immensely complex loop structure in the chromosphere and the corona, not only of the magnetically active areas but also of the quiet Sun. Loop oscillations and turbulence have been observed, as well as pre- and post-flare structures. The TRACE homepage (http://vestige.lmsal.com/TRACE/) offers a set of images and movies of these unique observations. 


\subsection{RHESSI: THE HIGH-ENERGY MISSION TO STUDY THE ACTIVE SUN}

The Reuven Ramaty High Energy Solar Spectroscopic Imager (RHESSI) is a NASA small Explorer (SMEX) mission with Swiss contributions. It was launched in February 2002 into a low-altitude orbit inclined $38^{\circ}$ to the Earth's equator. The only instrument on board is an imaging spectrometer with the ability to obtain time series of solar flares with high temporal resolution in $\mathrm{X}$ - and $\gamma$-rays. It uses two new complementary technologies: fine grids to modulate the solar radiation, and germanium detectors to measure the energy of each photon very precisely. Its primary mission objective is to study the basic physics of particle acceleration and energy release in solar flares. RHESSI will be operating until 2007. A description of the mission and its first results have been published in a special issue of Solar Physics (Vol. 210, 2002).

RHESSI has observed a large number of energetic flares and provided the first hard X-ray imaging spectroscopy of flares from thermal to nonthermal energies. Also, the first high-resolution flare $\gamma$-ray line spectrum was produced, measuring redshifts of a fraction of a percent and thus implying directivity of the energetic ions as well as non-radial magnetic fields. Performing the first imaging of flare $\gamma$-ray lines, RHESSI has given evidence that the centres of energetic ion and electron sources are separated by roughly $20^{\prime \prime}$. In addition to its solar results, RHESSI was able to detect strong polarisation in a cosmic $\gamma$-ray burst, implying strong coherent magnetic fields at the source. Some of its recent findings, especially on flares, are summarized in a special issue of the Astrophysical Journal Letters (Vol. 595, 2003).

\subsection{CORONAS: A SEQUENCE OF MISSIONS TO STUDY THE SOLAR CYCLE}

CORONAS, a project operated by the Russian space agency ROSAVIACOSMOS with international contributions, stands for "Complex Orbital Near-Earth Observations of Solar Activity" (in Russian). It comprises a sequence of three satellites each carrying a set of devices capable of obtaining data from $\gamma$ - and X-rays to radio waves. CORONAS-I, the first of the three planned observatories within the framework of the project, was launched in 1994, but was operational for only a few months. The second, CORONAS-F, was launched in July 2001. The satellites are placed into polar orbits at about $500 \mathrm{~km}$ and inclined at roughly $80^{\circ}$. The resulting quasi-synchronous orbit assures periods of recurrence of up to 20 days during which the satellite stays out of the Earth's shadow. The mission is aimed at the study of the internal structure of the Sun and solar flares. A description of the mission can be found in Novosti Kosmonavtiki (Space 
News, No.9, 2001; No.5, 2002, in Russian). The Spectroheliographic X-Ray Telescope (SPIRIT) has recorded images of high-temperature plasma (3-20 MK) in Mg XII and found compact sources at locations up to $0.3 R_{\odot}$ from the limb (Zhitnik et al., 2003). A third mission (PHOTON) is foreseen to follow the present one.

\section{Our present state of knowledge}

The availability of the new ground-based and space facilities described in Sections 3 and 4 has led to major progress in our understanding of the Sun. We briefly describe some of these most important findings. An excellent account of these achievements is also given by Lang (2000).

\subsection{THE SOLAR INTERIOR}

Helioseismology, observations from ground-based networks and from SOHO, in combination with the results from neutrino measurements, supplied corroborating evidence that the standard solar model is basically correct with a precision of a few parts per thousand. Comparisons between models and helioseismic inversions have been made by, e.g., ChristensenDalsgaard et al. (1996), Richard and Vauclair (1997), Turck-Chièze et al. (2001a) or Couvidat et al. (2003). Already early helioseismic results showed that the sound speed between 0.3 and $0.7 \mathrm{R}_{\odot}$ (below the convection zone) was higher than predicted by models, such that the assumed opacity in the models had to be re-assessed, as well as element settling and small mixing near that area (Brun et al., 1998, 1999; Vauclair, 1998; Turck-Chièze et al., 1998). A comparison of modelled and measured sound speed in the solar interior is shown in Figure 6. Gough and Scherrer (2001) give an overview of helioseismology results and their relevance for solar modelling. Using SOHO-MDI data Schou et al. (1998) calculated the solar rotation in the convection zone and in the outer part of the radiative core with unprecedented accuracy. Figure 7 shows a combination of results from MDI and ground-based data (LOWL and BiSON). The rotation of the core at distances less than $0.05 R_{\odot}$ is still uncertain because of the unavailability of modes penetrating deep enough in the core and in particular the g-modes. For the low-degree modes that reach down to the core, only a correspondingly limited azimuthal-order ( $m$-value) range is available and the splitting is hard to detect. Low- and intermediate-degree splittings have been estimated from a variety of instruments and different data analysis procedures. Therefore, the findings differ significantly (Eff-Darwich and Korzennik, 1998). Recent results support a rotation rate of the core which does not exceed the rotation at mid-latitudes by more than $20 \%$ (Eff-Darwich et al., 2002). Elsworth et al. (1995) inferred evidence for a slowly rotating core 


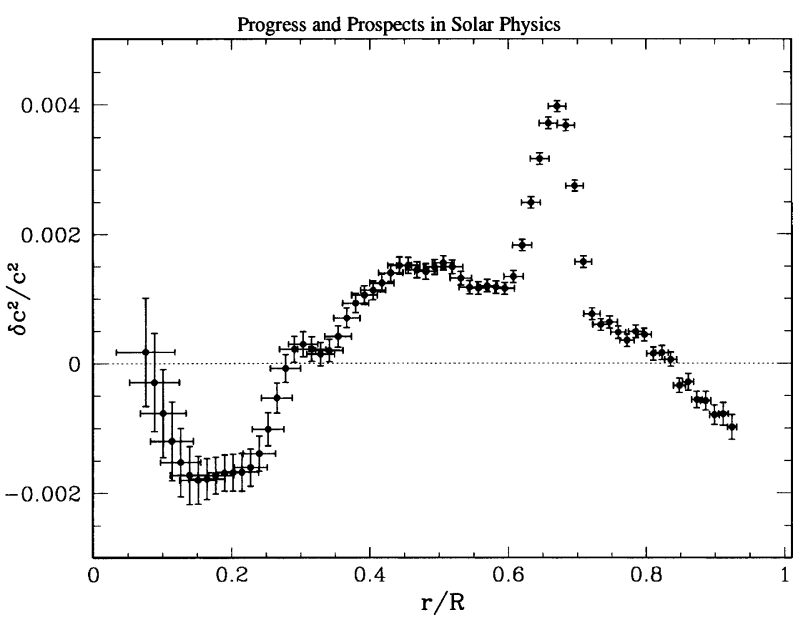

Figure 6. The relative differences between the squared sound speed in the interior of the Sun in a standard solar model (Christensen-Dalsgaard et al., 1996) and as inferred from 2 months of MDI data. The horizontal bars show the spatial resolution, and the vertical bars are the estimates of the uncertainty. Credit: SOHO/MDI.

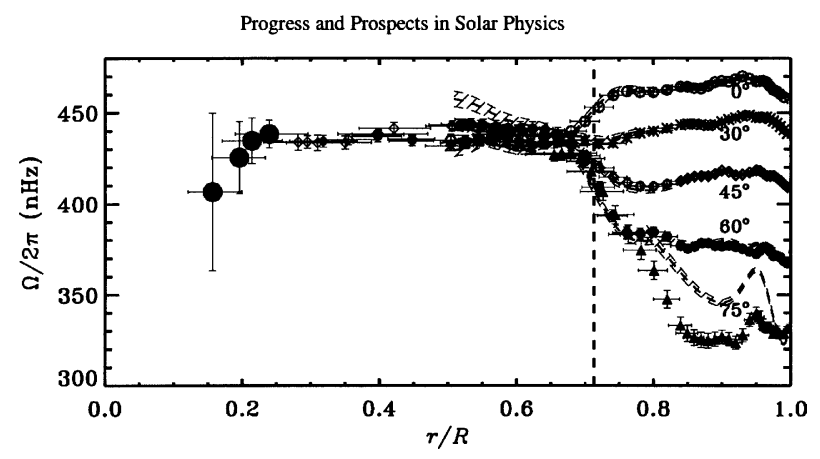

Figure 7. Rotation rate $\frac{\Omega}{2 \pi}$ as a function of fractional radius at different latitudes within the Sun. The results in the outer part for $r \geq 0.5 R_{\odot}$ were obtained with MDI (Schou et al., 1998), the results for $r \leq 0.45 R_{\odot}$ were obtained from a combination of BiSON and LOWL data (Chaplin et al., 1999). The tachocline - the region where the rotation rate changes from differential rotation in the convection zone to nearly solid-body rotation in the interior - is evident near the base of the convection zone, at roughly $0.71 R_{\odot}$. (Courtesy of M. Thompson).

from an analysis of BiSON data. The results obtained by Chaplin et al. (1999) from BiSON and LOWL are consistent with a constant rotation of the deep radiative interior at roughly the same (or a slightly lower rate) as the equatorial surface rate.

At the base of the convection zone, small (6-8 nHz peak-to-peak) oscillations in the inferred rotation rate have been observed by Howe et al. (2000). These oscillations show a periodicity of about 1.3 years. The cause 
of these variations near the tachocline is not yet understood. They might be related to variations in the angular momentum and with the exchange of angular momentum between the radiative and the convective zones (Komm et al., 2003) and might also include solar-cycle related trends (Toomre et al., 2003). This periodicity has also been found to be present in sunspot data, with a power that is highly time-variable (Krivova and Solanki, 2002). Longer time series are probably required to confirm these important findings.

\subsection{SUBSURFACE LAYERS}

In addition to global scale observations, spatially resolving instruments, like, e.g., MDI, allow local helioseismology techniques (Table I) to probe local sound speed variations and flows in the near surface regions just beneath supergranulation and sunspots. A variety of subsurface flow structures has been discovered using acoustic tomography techniques. Subsurface meridional flow from equator toward the poles was detected with MDI (Giles et al., 1997), reaching deep into the convection zone with a speed decreasing with depth. A return flux has not yet been discovered within the depths studied so far. This poleward flow can possibly contribute to the polarity reversal of the 22-years cycle in transporting magnetic remnants of sunspots from lower to higher latitudes (Duvall and Kosovichev, 2001; Kosovichev, 2003).

TABLE I

Basic methods of local-area helioseismology (Courtesy of A. Kosovichev)

\begin{tabular}{|c|c|c|}
\hline Method & Observable & Inferences \\
\hline $\begin{array}{l}\text { Ring-diagram analysis } \\
\text { (e.g., Hill, 1989, Haber } \\
\text { et al., 2002) }\end{array}$ & $\begin{array}{l}\text { Wavenumber-frequency } \\
\text { relation }\end{array}$ & $\begin{array}{l}\text { Radial variation of sound } \\
\text { speed and flows }\end{array}$ \\
\hline $\begin{array}{l}\text { Time-distance } \\
\text { techniques } \\
\text { (Duvall et al., 1993; } \\
\text { Kosovichev and Duvall, } \\
\text { 1997) }\end{array}$ & $\begin{array}{l}\text { Phase and group travel } \\
\text { times of acoustic and } \\
\text { surface gravity waves }\end{array}$ & $\begin{array}{l}\text { 3D sound speed density and } \\
\text { flows }\end{array}$ \\
\hline $\begin{array}{l}\text { Acoustic imaging } \\
\text { (Chou et al., 1991) }\end{array}$ & $\begin{array}{l}\text { Phase and amplitude } \\
\text { variations }\end{array}$ & 3D sound speed and flows \\
\hline $\begin{array}{l}\text { Helioseismic } \\
\text { holography (Lindswy } \\
\text { and Braun, 1990, 2000) }\end{array}$ & $\begin{array}{l}\text { Phase and amplitude } \\
\text { variations }\end{array}$ & 3D sound speed and flows \\
\hline
\end{tabular}


More complex flows have been deduced below the solar surface, exhibiting a structure closely related to surface features like magnetic active regions and supergranulation (Kosovichev, 1996; Duvall and Gizon, 2000). The flows seem to interact with the surface magnetic field, displaying a pattern of divergence and convergence, with active regions appearing as zones of convergence and possible subduction. This suggests that the subsurface flows might mechanically interact with the field lines, leading to unstable magnetic configurations that may flare or erupt as coronal mass ejections. By continuous observation of the variable flow pattern, the interaction of the large-scale flows and magnetic fields can be studied. It might then become possible to establish a relation in order to predict solar activity manifestations (Kosovichev, 2003).

The flows beneath very active regions and sunspots have been studied in detail with MDI (Kosovichev et al., 2000; Zhao et al., 2001). Figure 8 shows a vertical section of the sound speed below a spot. The perturbations of the magnetosonic sound speed are associated with the magnetic field and temperature variations. The sound speed perturbations under spots are much stronger than those corresponding to the average emerging flux. Beneath the spot, the perturbation is negative in the subsurface layers.

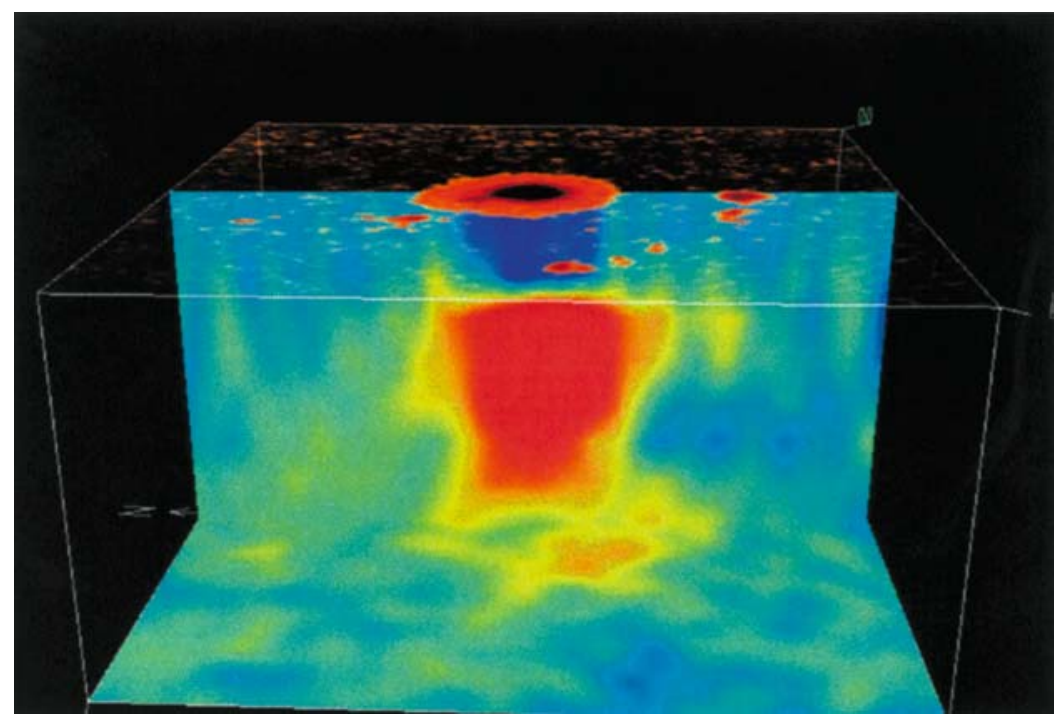

Figure 8. The subsurface structure (sound speed) below a sunspot as derived from MDI measurements, using the technique of time-distance helioseismology. Three planes are shown. The surface intensity shows the sunspot with the dark central umbra surrounded by the somewhat brighter, filamentary penumbra. The second plane cuts from the surface to $24000 \mathrm{~km}$ deep, showing areas of faster sound speed as reddish colours and slower sound speed as bluish colours. The third plane (bottom) is a horizontal cut at a depth of $2200 \mathrm{~km}$ showing the horizontal variation of sound speed. Image courtesy of A. Kosovichev. 
Deeper, it becomes positive. The negative perturbations beneath the spot might be due to a lower temperature. It is shown that the magnetic inhibition of convection, that makes sunspots cooler, is most effective within the top 2 to $3 \mathrm{Mm}$ of the convection zone. However, the separation of the effects of the temperature and the magnetic field on the velocity is an important problem of solar tomography to be solved in the future (Kosovichev, 2003).

Holography and time-distance methods also provide a new approach for the prediction of the emergence of new active regions on the far side of the Sun (Lindsey and Braun, 2000; Duvall and Kosovichev, 2001). This would prove to be extremely useful for space weather forecasts, i.e., the prediction of solar influences on the Earth.

\subsection{SOLAR MAGNETISM AND SOLAR VARIABILITY}

When the Ulysses spacecraft passed over the South pole of the Sun, at a distance of about 2.2 AU below the ecliptic plane, in October 1994, for the first time the topology of the magnetic field that had been suggested by Parker (1958) could be probed. Analyzing Ulysses radio-data measurements, Reiner et al. (1995) were able to track several solar type III radio bursts through interplanetary space, clearly revealing the global spiral magnetic field topology.

With improved solar observations also, more and more of the complexity of the Sun's magnetic field was revealed. Not only do the large scale features like coronal hole structures and sunspots, but also diffuse small scale patterns, play an important role in the generation and distribution of the field (Choudhuri, 2003). Using data from MDI, Schrijver et al. (1998) discovered that the Sun is covered with a small-scale magnetic structure, consisting of positive and negative magnetic fragments continually emerging, cancelling, merging and fragmenting, thus replacing the magnetic flux roughly once every $40 \mathrm{~h}$. The magnetic field accumulates at the boundaries of the supergranule cells. The complex distribution and mixing of magnetic polarities over the entire Sun (see Figure 9) has been termed the "magnetic carpet". The magnetic loops, connecting regions of opposite magnetic polarity, rise far into the solar corona, forming extensive arch structures. While the bright active regions have long been known to be local sources of heating, the more diffuse and permanent heating appears to be associated with this ubiquitous magnetic carpet.

The total solar irradiance (TSI) or solar constant has been monitored since early space missions. A composite of measurements conducted since 1978 from various space missions is shown in Figure 10 (Fröhlich and Lean, 1998; Fröhlich, 2000). Details of the delicate calibration procedures are given in Fröhlich (2003). Within the uncertainties, no significant trend 


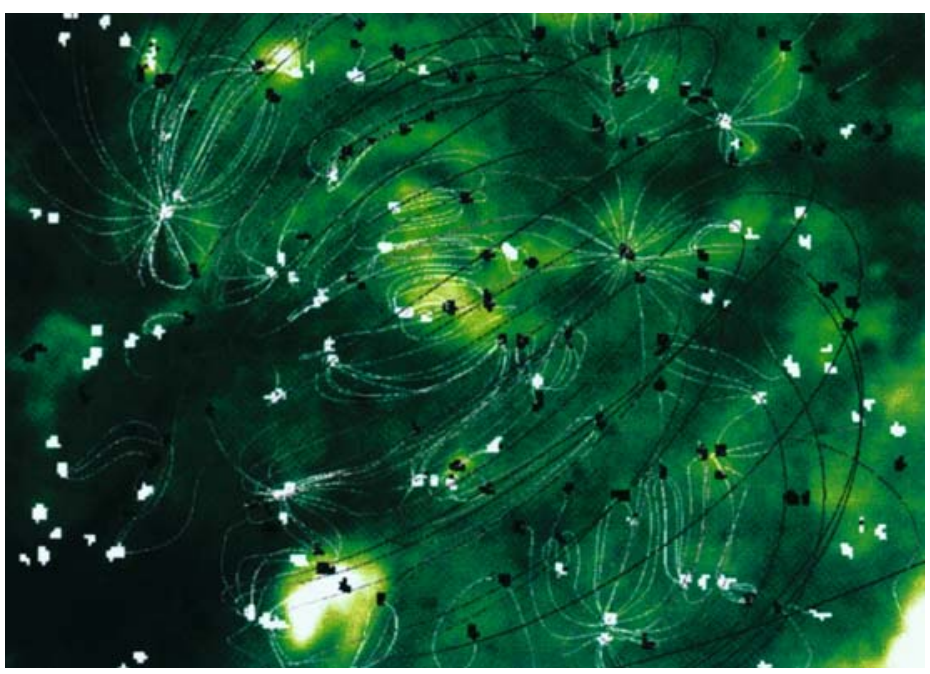

Figure 9. Shown here is a model of magnetic field lines at the surface of the Sun (also called the "magnetic carpet") developed using data from several SOHO instruments. The green and white plane is in image at coronal temperatures from the Extreme Ultraviolet Imaging Telescope (EIT) instrument. The image shows different amounts of coronal material at a temperature of about $2.7 \times 10^{6} \mathrm{~K}$. Whiter areas represent more material at this temperature, darker areas represent less. The black and white spots represent magnetic field concentrations with opposite polarity. Each spot is roughly $8000 \mathrm{~km}$ across. The width of the image is approximately $4 \times 10^{5} \mathrm{~km}$. Credit: Stanford-Lockheed Institute for Space Research and NASA Goddard Space Flight Center.

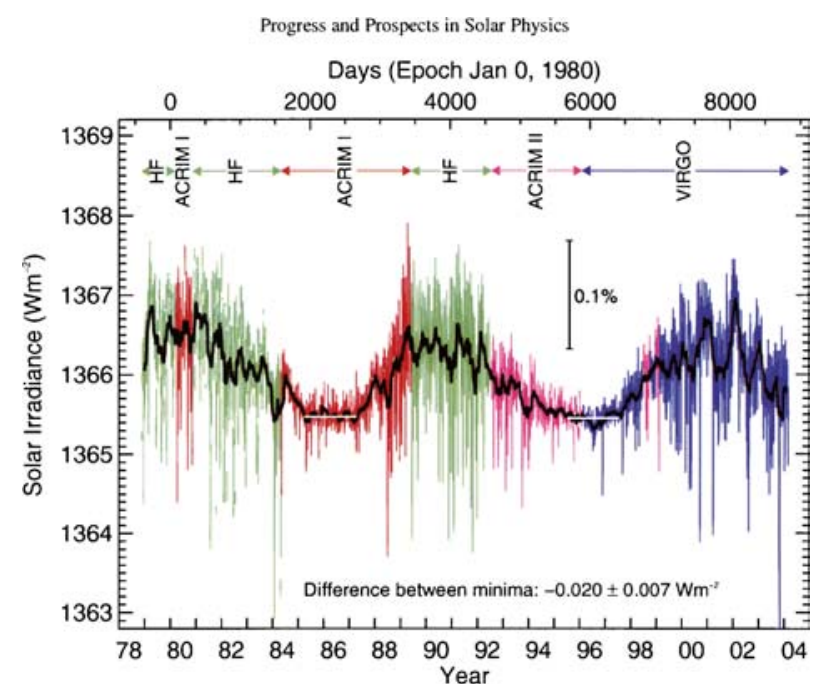

Figure 10. The total solar irradiance (TSI, daily values) as composed from various space missions. Also shown is an 81-day running mean. Image courtesy of C. Fröhlich. 
over the past 23 years can be deduced in the solar constant. The fast "noisy" variability observed during this time frame can precisely be attributed to the presence of spots and faculae on the disk (Solanki and Fligge, 2000). The amplitude of the solar irradiance variations over the 11-year cycle has been roughly $0.1 \%$ peak to peak. Although these variations seem small they are of the same order of magnitude as longer period insolation changes that lead to the formation of pronounced cold and warm periods (ice ages, etc.). However, to date it has not yet been possible to conclude definitely to what extent the total solar radiation variations have influenced the Earth's climate (see also Section 6.2).

A critical point in understanding the mechanisms that determine the solar cycle and the corresponding polarity change is the replacement of the old-cycle magnetic flux with the new-cycle flux of opposite polarity. Global flux observations have shown that the total magnetic flux on the Sun varies by about a factor of three between minimum and maximum activity, i.e., two orders of magnitude lower than the variation expected from the observed rate of flux emergence (Alexander and Acton, 2001). This means that the flux removal rate must account for the difference, and thus nearly match the emergence rate through the solar cycle. The flux removal can occur either via the submergence of magnetic fields or by the reconnection and expulsion of magnetic flux into the interplanetary medium, e.g., by CMEs. Recent results of Gopalswamy et al. (2003) support the latter process.

\subsection{THE SOLAR ATMOSPHERE}

The instruments on SOHO and TRACE have provided a wealth of new discoveries, thanks to the high temporal and spatial resolutions in the EUV wavelength range. It is now clear that there is no such thing as a perfectly quiet solar atmosphere. Instead, with improving solar instrumentation, higher variability and increasing complexity on all scales is revealed.

\subsubsection{The photosphere, chromosphere and transition region}

Certainly, great progress in our knowledge of the photosphere has come from improved ground-based observations, especially with the advent of adaptive optics techniques (cf., Section 3.1). However, space-based instrumentation has made it possible to acquire long uninterrupted time series. This is particularly important for spectral analyses of the larger scale velocity fields induced by convection (Nordlund, 2003). Gizon et al. (2003) reported observations showing that supergranulation, i.e., the pattern of horizontal outflows, outlined by a network of small magnetic features, with a distinct scale of 30 million metres and an apparent lifetime of one day, undergoes oscillations and supports waves with periods of 6-9 days. Using 
MDI dopplergrams Beck et al. (1998) finally discovered the largest signature so far of convection, giant cells, covering $40^{\circ}$ to $50^{\circ}$ in longitude and $7^{\circ}$ to $8^{\circ}$ in latitude, with a velocity of $3.6 \mathrm{~m} / \mathrm{s}$.

On SOHO, the CDS (Coronal Diagnostic Spectrometer, Harrison et al., 1995) and SUMER (Solar Ultraviolet Measurements of Emitted Radiation, Wilhelm et al., 1995) spectrographs have, thanks to their unprecedented sensitivity, detected many new lines in the far and extreme ultraviolet that were too weak to be measured earlier.

The EUV irradiance spectrum covering the ranges $30.7-38.0 \mathrm{~nm}$ and 51.5-63.2 $\mathrm{nm}$ has been measured by CDS (Brekke et al., 2000). Curdt et al. (2001) have compiled a spectral atlas from SUMER measurements in the wavelength range between 67.0 and $160.9 \mathrm{~nm}$. Figure 11 shows part of the spectrum.

With its imaging capabilities, the Extreme-ultraviolet Imaging Telescope (EIT, Delaboudinière et al., 1995) on SOHO, which observes the Sun in four selected EUV bandpasses (Table II), is a unique tool to study the chromosphere, transition region and inner corona throughout the solar

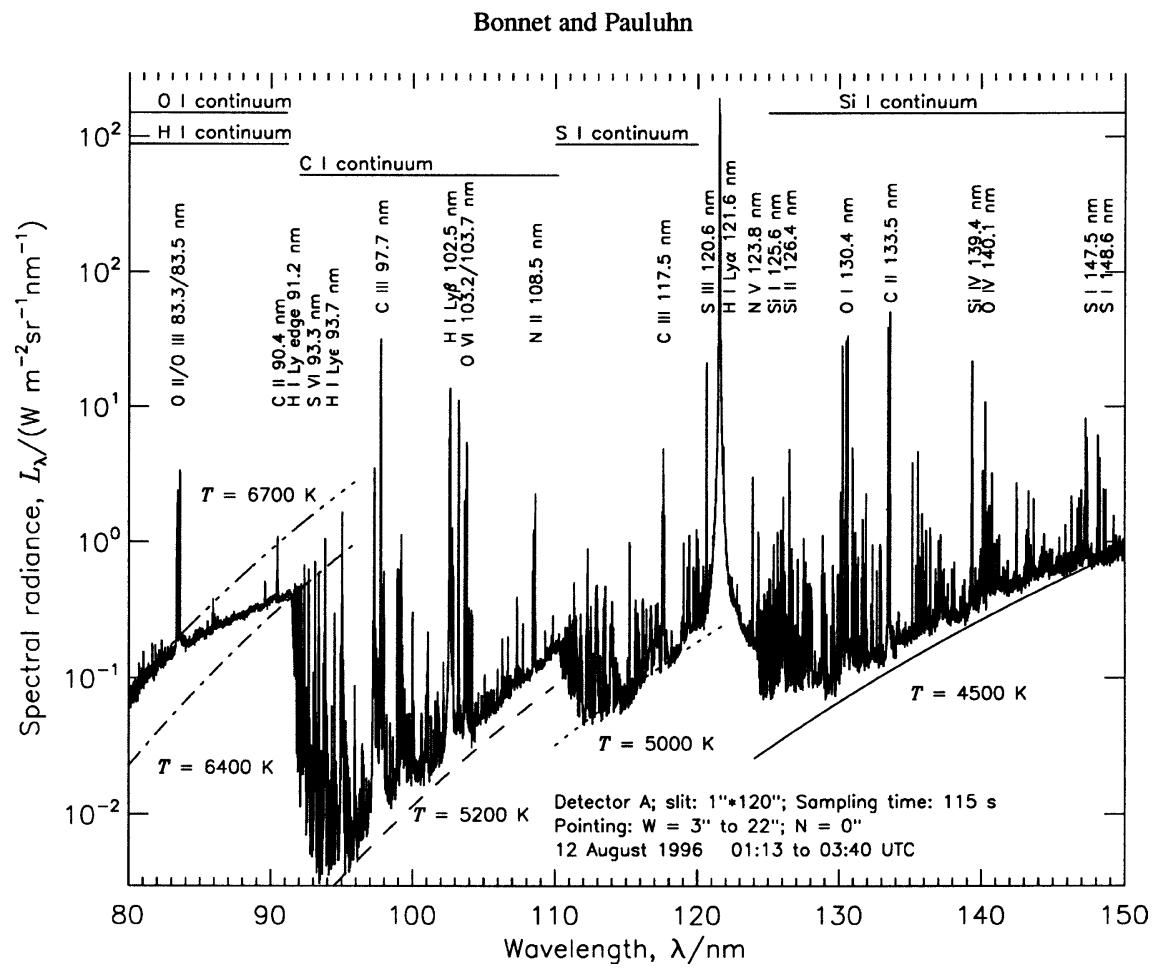

Figure 11. SUMER radiance spectrum of a quiet-Sun region. Prominent lines and continua are identified. Some black-body radiation levels are shown to provide an estimate of the radiation temperatures of the continua. (Courtesy of K. Wilhelm). 
TABLE II

Wavelengths and temperatures measured by EIT

\begin{tabular}{lll}
\hline Wavelength & Dominant ion & Characteristic temperature \\
\hline $30.4 \mathrm{~nm}$ & He II & $8 \times 10^{4} \mathrm{~K}$ \\
$17.1 \mathrm{~nm}$ & Fe IX/X & $9 \times 10^{5} \mathrm{~K}$ \\
$19.5 \mathrm{~nm}$ & Fe XII & $1.3 \times 10^{6} \mathrm{~K}$ \\
$28.4 \mathrm{~nm}$ & Fe XV & $2 \times 10^{6} \mathrm{~K}$ \\
\hline
\end{tabular}

cycle (Figure 12). A remarkable set of heliograms obtained with SOHO in other chromospheric lines has been published in the SUMER atlas (Feldman et al., 2003). A discussion of several chromospheric features, like, e.g., spicules, which are important because they intermittently connect the lower parts of the atmosphere with the corona, is given by Peter (2002).

Heating mechanisms for the chromosphere are discussed by Narain and Ulmschneider (1996) and Ulmschneider and Kalkofen (2003). They conclude that the most probable scenario is a combination of acoustic and magneto-hydrodynamic (MHD) waves. The base of the chromosphere is

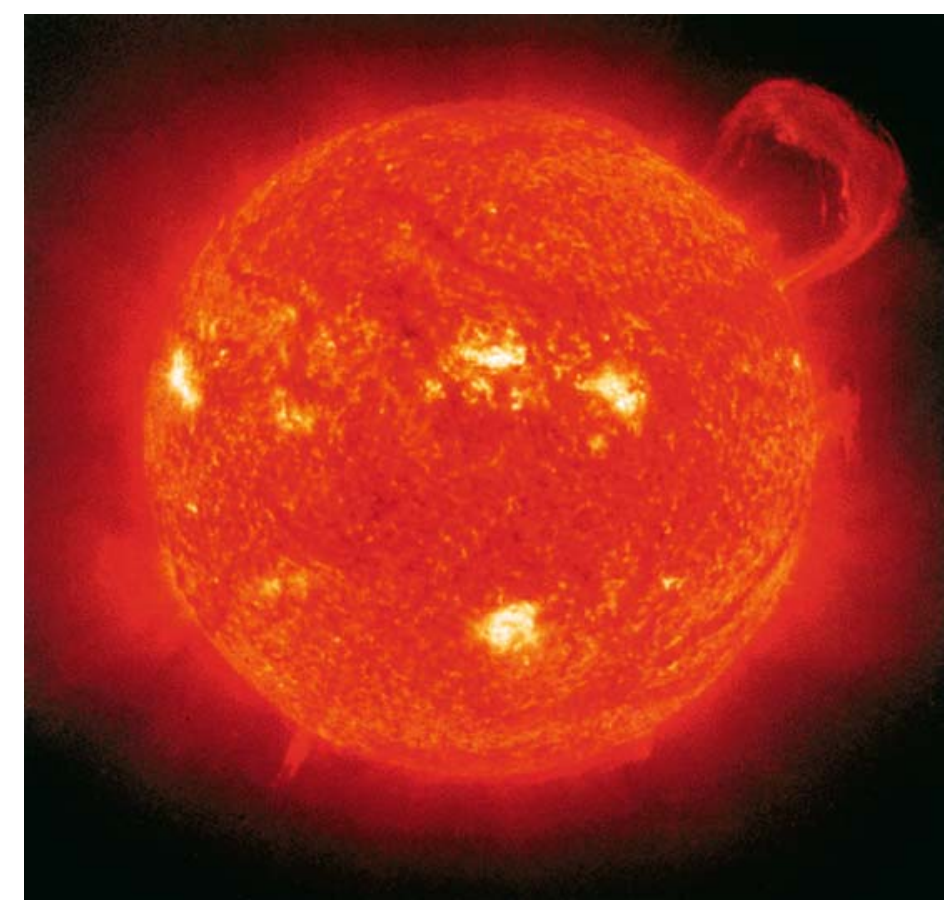

Figure 12. EIT $30.4 \mathrm{~nm}$ image of a huge prominence, i.e., relatively cool dense plasma suspended in the hot, thin corona, taken on September 14,1999. Credit: EIT/SOHO. 
heated by pure acoustic shock waves. The lower and middle parts, where the relative importance of the magnetic flux tubes increases, are heated by longitudinal MHD shock waves propagating in the vertically oriented flux tubes which spread with height and eventually fill the entire available space. In the upper parts of the chromosphere non-wave magnetic processes like (reconnective) micro- or nanoflares may also contribute to the heating. However, the structure and dynamics of the chromosphere are still a matter of debate and intense study. The traditional view of a relatively homogeneous thermal structure has long been given up (e.g., Ayres et al., 1986; Solanki et al., 1994; Carlsson and Stein, 1995), and a morphological characterisation based on its magnetic structure might be a better description (Wilhelm and Kalkofen, 2003). Recently, a technique involving analysis of radio observations (at millimeter and submillimeter wavelengths) that are more sensitive to dynamic processes in the chromosphere has been suggested (Loukitcheva et al., 2004). Thus, future high-resolution radio observations, for example with the Atacama Large Millimeter Array (ALMA) (http://www.alma.nrao.edu), may be particularly useful in determining the nature of the solar chromosphere.

In the transition region, observations from SOHO and TRACE have revealed a finely structured, highly dynamic and extremely time variable plasma region, structured by the magnetic field.

In the lines formed in the lower and cooler parts of the transition region, the extension of the chromospheric network is prominent. Beyond $2.5 \times 10^{5} \mathrm{~K}$ the network widens rapidly with increasing temperature and becomes increasingly diffuse. The emission from loops at transition region temperatures is extremely time variable and features intensity changes at time scales of minutes (Kjeldseth-Moe, 2003).

Transient events on all temporal and spatial scales have been detected at transition region temperatures, and the zoo of variable events has been expanded to include phenomena like flares of all sizes, blinkers (i.e., localised brightenings lasting some tens of minutes), and explosive events (Alexander and Acton, 2001; Švestka, 2003).

Flaring events caused by magnetic reconnection are observed on a scale from large flares to nanoflares, spanning several orders of magnitude in the energy spectrum. Explosive events during which gas is ejected in a bidirectional jet with speeds up to $150 \mathrm{~km} / \mathrm{s}$ (Innes et al., 1997) have also been interpreted as being due to reconnection. Blinker-type events (Harrison, 1997; Brković et al., 2000), however, have been interpreted as density enhancements and not as direct effects of reconnection.

There are many indications that significant variations occur on spatial and temporal scales that are smaller than can be resolved by today's instruments. Clearly, observing the transition region zone poses the highest demands on spatial and temporal resolution for future instrumentation. 


\subsubsection{The highly structured corona}

EUV observations confirm that the corona, like the transition region, consists mainly of loop structures. Active region loops in the corona reach temperatures of 2 to $5 \times 10^{6} \mathrm{~K}$ during their quiet phases and up to around $10^{7} \mathrm{~K}$ during heating bursts. The heating of coronal loops is most probably of magnetic origin. Footpoint motions in the photosphere generate waves that travel upwards along the magnetic field. Different types of waves exist, such as kink waves along the flux tubes, torsional waves, and longitudinal (or sausage mode) waves. Several more modes are possible, e.g., along the outside of thick flux tubes, or in the interior (Solanki and Hammer, 2001). The location of the loop heating function had been suspected to be either uniform along the loop or at the top of the loop, until Aschwanden (2001) showed that footpoint-heating could match the observations well. It is now envisaged that the spatial heating function is concentrated in the lowest $\leq 20 \mathrm{Mm}$ of each coronal loop (Aschwanden, 2002). This location points towards a heating mechanism operating at chromospheric and transition region heights.

Loops might be made up of several threads, all with their own energy balance and temperature profile (Reale and Peres, 2000). Unfortunately, their full spatial and temporal variability is yet unresolved. The observational averaging effect might explain why Yohkoh, which samples a broader temperature range, has observed in general thicker loops than TRACE (Peres, 1999). TRACE has indeed observed a braiding and twisting of individual loop threads (Schrijver et al., 1999). If this braiding is strong enough, it may contribute to coronal heating via electric currents or reconnection events. Figure 13 shows a TRACE image of a coronal loop structure.

Recently, oscillations of loops could be measured with imaging observations from SOHO and TRACE. This leads to the relatively new technique of "coronal seismology", originally suggested by Uchida (1970) and Roberts et al. (1983), probing the corona with standing MHD waves and oscillations (Nakariakov and Ofman, 2001; Nakariakov, 2003). Several oscillation modes have been observed with TRACE (Aschwanden et al., 1999; Schrijver et al., 2002), SUMER (Wang et al., 2002, 2003), or radioheliographs (Asai et al., 2001; Aschwanden et al., 2004). Observing the loop oscillations, which are most probably triggered by nearby flares or erupting filaments, and measuring their oscillation characteristics, allows the determination of coronal plasma parameters which are otherwise not accessible, for example, the viscosity, resistivity and thermal conduction. All these coronal transport coefficients must play a crucial role in coronal heating and are still largely unknown. Nakariakov et al. (1999) determined the damping of waves in coronal plasma and thus its viscosity, and found it larger than expected, which they ascribed to coronal micro-turbulence. 


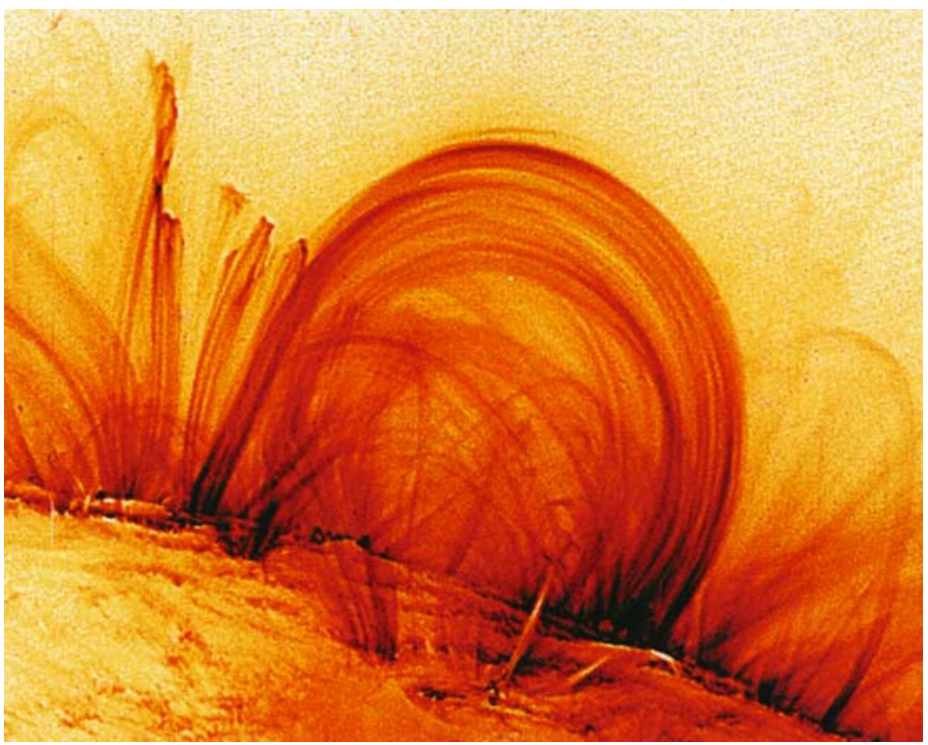

Figure 13. This image of coronal loops over the eastern limb of the Sun was taken in the TRACE $17.1 \mathrm{~nm}$ pass band, characteristic of plasma at $1 \mathrm{MK}$, on 6 November 1999. Credit: TRACE, Stanford-Lockheed Institute for Space Research.

This might indicate that the resistivity in the corona is at an enhanced level and thus the dissipation of MHD waves or magnetic reconnection could proceed much faster than expected. This would eliminate some of the difficulties presently encountered in solving the coronal heating problem.

As we can see, coronal heating is a highly complex problem because of the variety of structures present in the corona, and it is most probable that several mechanisms and processes are involved. Handy and Schrijver (2001), using EIT and MDI data, have shown that the coronal pattern over the quiet Sun decorrelates completely on a time scale of no more than $15 \mathrm{~h}$ which agrees well with the timescales of around $40 \mathrm{~h}$ observed in the "magnetic field carpet". Only waves where the magnetic field provides the restoring forces seem able to heat the corona. Due to limited-telemetry reasons, MHD waves with very short periods cannot yet be observed with space-based instrumentation. These, however, are the most promising candidates to contribute to coronal heating (Phillips and Dwivedi, 2003). Ground-based observations with high speed CCD instrumentation during eclipses and with coronagraphs might provide the required measurements (Pasachoff and Ladd, 1987; Williams et al., 2002) but will lack the long time series.

A number of observations suggest that small-scale transient events might play an important role in heating the solar corona. TRACE has observed flares on scales seven to eight orders of magnitude lower than 
that of "ordinary" flares. Small-scale flaring events (such as nanoflares or picoflares) might even be powerful enough to provide a significant source of coronal heating if they are numerous enough, i.e., if their relative energy input, compared with larger flares, is large enough (Hudson, 1991). This issue of the flare distribution, however, is still controversial (Krucker and Benz, 1998; Benz and Krucker, 2002; Parnell and Jupp, 2000; Aschwanden and Parnell, 2002). Nevertheless, even if nanoflares cannot be the main cause of coronal heating, the energy input of the observed quiet-Sun transients would be enough to power the quiet solar corona, and the solar quiescent emission might simply be made up of unresolved flares (Phillips and Dwivedi, 2003; Pauluhn and Solanki, 2004). Marsch et al. (2003) suggest a heating by high-frequency waves resulting from picoflares already occurring in the supergranular network.

Of major importance for coronal physics is better measurement of the magnetic field in chromosphere and corona. Recent progress has been made in measuring the full magnetic vector in an interaction region near the base of the solar corona (Solanki et al., 2003). Clearly, there is room for further observations in this field.

\subsubsection{The fast and slow solar wind}

The structure of the solar wind was studied by in situ particle instruments, especially those on board Ulysses. Geiss et al. (1995) confirmed the basic differences of the two quasi-stationary types of solar wind: they differ in their heliospheric properties, as well as in their chromospheric and coronal properties, namely in speed, elemental composition, and charge-state composition. A sharp boundary separates the two types through all these regions. Von Steiger et al. (2000) concluded that slow and fast wind appear in regions of different coronal temperatures. All parameters are found to be more variable in the slow solar wind. Figure 14 shows a superposed picture of the solar disk and corona together with Ulysses measurements. The coronal holes as regions of emergence of the fast steady wind, and the coronal streamers (i.e., the helmet and ray-shaped structures near the solar equator) as regions of emergence of the slow wind, can be well identified.

The outflow from network boundaries, or intersections of boundaries, in the higher temperature regions of the transition region and especially in coronal holes where the large-scale magnetic field is open, has been identified with the emergence of the fast wind from coronal holes (Hassler et al., 1999; Peter and Judge, 1999; Stucki et al., 1999; Wilhelm et al., 2000). Coronal holes are structured by plumes, i.e., ray-shaped features that can be traced out to several $R_{\odot}$. They have higher densities and lower temperatures than the interplume regions. Model calculations together with SUMER observations (Wilhelm et al., 1998) have shown that the outflow speeds are much lower within plumes, so that interplume regions are prob- 


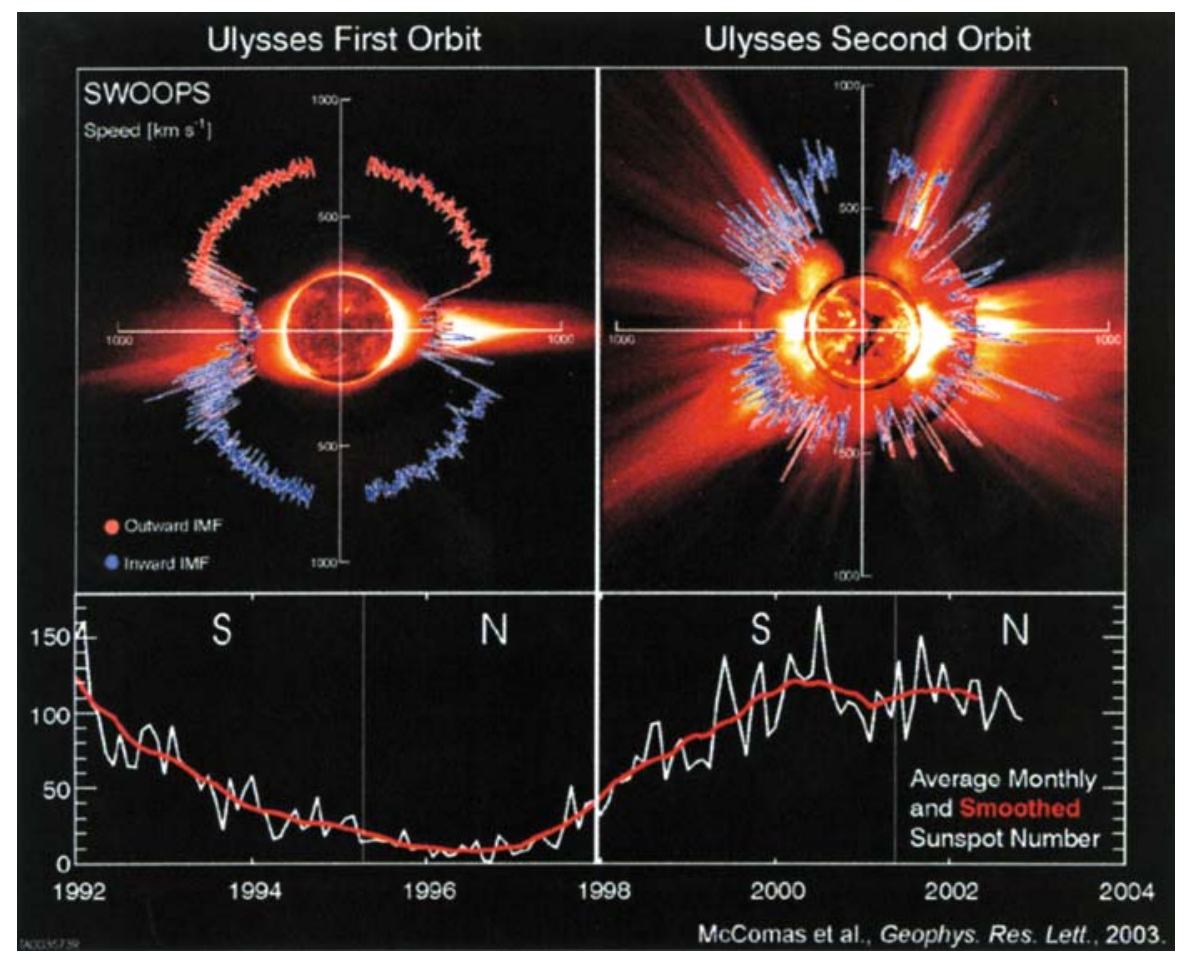

Figure 14. The solar wind as measured by the Ulysses SWOOPS (Solar Wind Observations Over the Poles of the Sun) instrument. Shown are the velocities of the solar wind (in $\mathrm{km} \mathrm{s}^{-1}$ ) overlaid over images from EIT/SOHO. The direction of the interplanetary magnetic field (IMF) is denoted by red and blue colours, respectively. The first orbit of Ulysses took place during solar minimum conditions, the second during solar maximum (also compare the corresponding plot of the sunspot numbers below the images). From McComas et al. (2003).

ably the genuine regions of emergence of the fast solar wind. This seems to be confirmed by UVCS (Ultraviolet Coronagraph Spectrometer, Kohl et al., 1995) measurements (Giordano et al., 2000). The plasma that makes up the fast solar wind would flow first through open funnels within the network and would then be accelerated within the low-density interplume lanes. Most probably, the fast wind is to a large part sustained through high-frequency wave dissipation, already in the lower solar corona (Marsch et al., 2003). The result that the transverse ion speed is up to a factor of 100 larger than the longitudinal speed (relative to the magnetic field direction) and its interpretation in terms of ion-cyclotron waves was one of the major breakthroughs of UVCS on SOHO (e.g., Cranmer et al., 1999). Cranmer (2000) investigated the ion-cyclotron wave dissipation of Alfvén waves using data from more than 2000 ion species measured by UVCS. He found that the high-frequency waves are already damped very efficiently when they resonate with ions of relatively small charge-to-mass ratios in 
the lower corona. Recently, these results have been subject to discussion, when Raouafi and Solanki (2004) showed that the velocity anisotropy is strongly dependent on the adopted density profile.

Our knowledge of the slow solar wind is less advanced. It is by far more irregular and filamentary than the fast wind. The majority of the slow wind (roughly 80 to $90 \%$ ) connects to variable fields at the coronal base in the magnetic network and comes from the streamer belt. The other fraction of the slow wind has no connection to the coronal base. It comes in the form of small plasmoids or bubbles (Sheeley et al., 1997), and seems to originate from bursty reconnection processes, mostly at points of field reversals at the top of coronal streamers. A compilation of the properties of the solar wind types can be found in Marsch et al. (2003).

\subsubsection{Coronal mass ejections}

Another dynamic structure of the corona are the CMEs (Crooker et al., 1997). They are now known to play one of the most important roles in solar-terrestrial relations. CMEs are due to the disruption of closed magnetic structures along a huge part of the solar surface. Their mass ranges generally from $10^{15}$ to $10^{16} \mathrm{~g}$. Their fronts propagate through interplanetary space with speeds between 100 and $1000 \mathrm{~km} / \mathrm{s}$, the average being $400 \mathrm{~km} / \mathrm{s}$ as was shown with the Large Angle Spectroscopic Coronagraph (LASCO, Brueckner et al., 1995). The precise relation between flares and CMEs is not yet clear. Some CMEs are preceded by flare eruptions; some are not. They occur both separately and together, in any combination. While flares and CMEs are observationally very different, the physics of both phenomena is similar: a sudden reconfiguration of the magnetic field releasing energy in the solar corona. However, various CME types appear to be very different in shape and structure, depending on the magnetic configuration of their generation site. Figure 15 shows a composite image of a $\mathrm{CME}$ obtained by combining two $\mathrm{SOHO} / \mathrm{LASCO}$ images and a $\mathrm{SOHO} /$ EIT image.

\section{The solar influence}

The Sun strongly influences our planetary system. How far this influence goes, and to what extent and on what timescales our own planet is affected, is the subject of intense research nowadays.

In addition to the variable radiative solar output, the fast and slow wind send streams of plasma and particles into the interplanetary medium (IPM) and coronal mass ejections emit plasma material and shock waves into space eventually to cause other particle acceleration there. All this produces highly variable and complex conditions in the IPM between the 


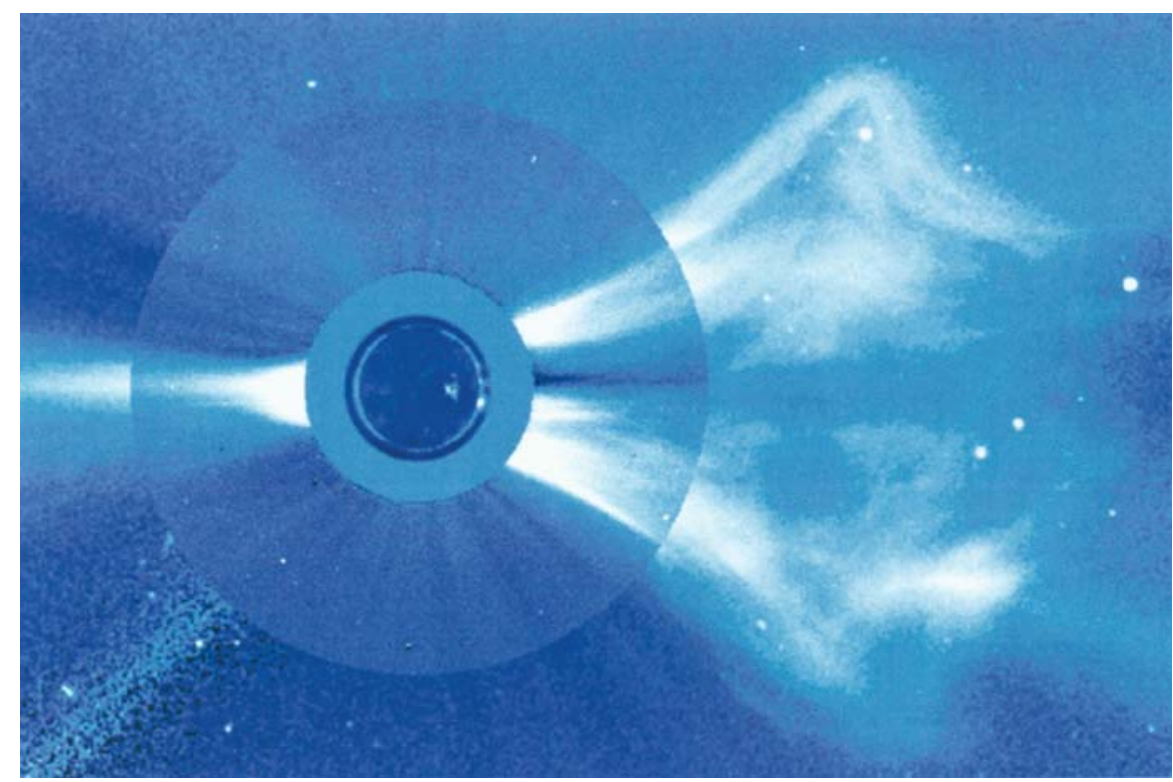

Figure 15. A composite of three images of a large CME. The images are from EIT (the blue Sun in the centre) and the LASCO C2/C3 instruments. The outermost circle centred on the Sun has a radius of approximately $32 R_{\odot}$. Credit: EIT and LASCO/SOHO.

Sun and the Earth, especially during periods of high solar activity. In the last decade, the term space weather was created to describe these conditions, stimulating a continuous monitoring of our star. Space weather mainly refers to short-term variability, but also long-term variations can have significant impact on terrestrial conditions. The main ways in which the Sun impacts the Earth are summarised in Table III. An excellent overview of this topic is given by Lockwood (2004).

\subsection{SPACE WEATHER}

One of the first associations of solar activity with geomagnetic storms was made as early as 1859 when Carrington and Hodgson, two British amateur astronomers, observed an intense white light flash on the Sun and linked it to geomagnetic disturbances observed several hours later (Carrington, 1859; Hodgson, 1859). These disturbances were of minor consequences then. This, however, has changed dramatically with our modern society being more and more dependent on electronic systems sensitive to solar perturbations and related geomagnetic effects, like electric power lines, pipelines, railways, radio communications, and of course artificial satellites. The impacts of the variable conditions in the Earth-related space environment, as well as recent developments in space weather research are described, e.g., in the volumes edited by Daglis $(2001,2004)$. 
TABLE III

The main ways in which solar variability influences the Earth

\begin{tabular}{lll}
\hline Solar output and time scales & Mechanism & Terrestrial impact \\
\hline $\begin{array}{l}\text { Variation of the total } \\
\text { solar irradiance } \\
\text { (years, decades, centuries) }\end{array}$ & $\begin{array}{l}\text { Changes in tropospheric } \\
\text { heating, changes in, } \\
\text { e.g., } \mathrm{O}_{3}, \mathrm{H}_{2} \mathrm{O} \\
\text { photochemistry }\end{array}$ & $\begin{array}{l}\text { Changes in the energy balance } \\
\text { and atmospheric circulation, } \\
\text { changes in atmospheric } \\
\text { composition }\end{array}$ \\
$\begin{array}{l}\text { Variation of the UV and } \\
\text { EUV irradiance } \\
\text { (months, years, decades, } \\
\text { centuries) }\end{array}$ & $\begin{array}{l}\text { Changes in } \\
\text { atmospheric } \\
\text { photochemistry, } \\
\text { e.g., } \mathrm{N}_{2}, \mathrm{O}_{2}, \mathrm{H}_{2} \mathrm{O}\end{array}$ & $\begin{array}{l}\text { Changes in the atmospheric } \\
\text { composition }\end{array}$ \\
$\begin{array}{l}\text { Shanges in the open } \\
\text { magnetic field leading } \\
\text { to changes in cosmic } \\
\text { ray flux at Earth } \\
\text { (months, years, decades, } \\
\text { centuries) }\end{array}$ & $\begin{array}{l}\text { Changes in concentration } \\
\text { of condensation nuclei } \\
\text { for cloud droplets }\end{array}$ & $\begin{array}{l}\text { Changes of cloud coverage of } \\
\text { low-lying clouds, changes in } \\
\text { the energy balance }\end{array}$ \\
$\begin{array}{l}\text { CMEs, flares, with } \\
\text { emission of energetic } \\
\text { particles (tens of } \\
\text { minutes, hours, days) }\end{array}$ & $\begin{array}{l}\text { Changes in upper } \\
\text { atmospheric ionisation } \\
\text { processes }\end{array}$ & $\begin{array}{l}\text { Short-term changes in } \\
\text { atmospheric photochemistry, } \\
\text { disruption of electronic } \\
\text { systems on Earth, aurorae }\end{array}$ \\
\hline
\end{tabular}

When a flare appears on the Sun, it is a source of X-rays which influence the Earth's ionosphere. The most energetic flares emit high-energy protons (exceeding $500 \mathrm{MeV}$ ) that arrive at Earth some 15 min later, producing neutrons in the the terrestrial atmosphere and causing ground level effects.

Charged particles of lower energies are guided by the terrestrial magnetic field to the polar caps causing aurorae. All these effects occur for tens of minutes to several hours after the flare onset, depending on the energy of the propagating particles. A TRACE image of the site of the largest solar flare observed to date is shown in Figure 16.

The major part of the effects on the Earth's magnetosphere is now attributed to the CMEs, which are the causes of geomagnetic storms. When their speed is high enough relative to the ambient solar wind, they are accompanied by a shock wave. The most geoactive CMEs originate in the central parts of the disk. They can originate in all parts of the Sun, also in quiet areas (Švestka, 2003). 


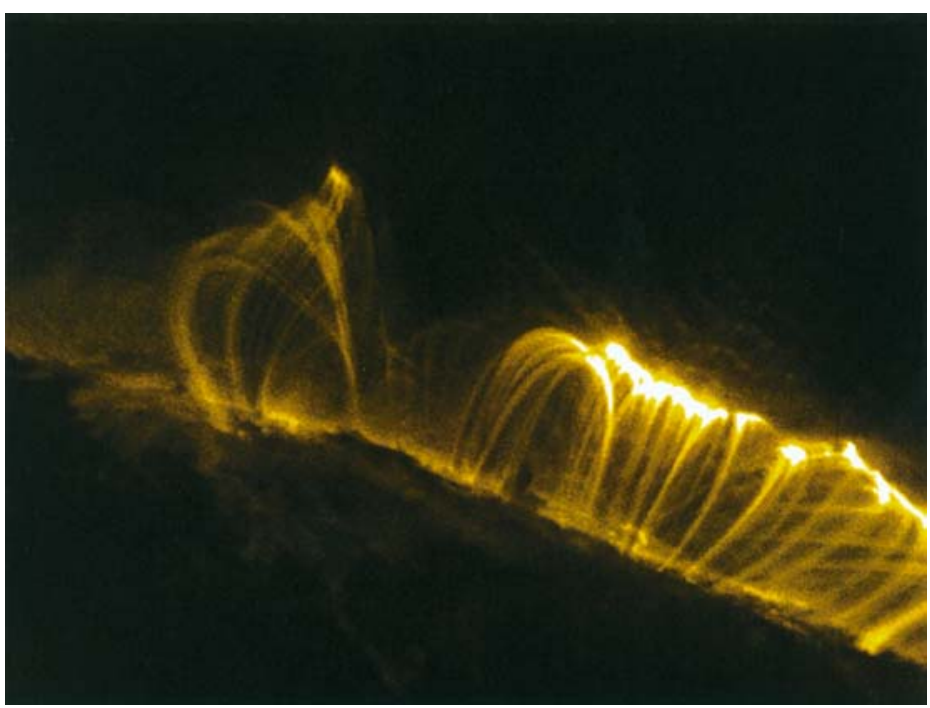

Figure 16. Huge post-flare loop observed on 4 November 2003. Credit: TRACE, StanfordLockheed Institute for Space Research.

Due to the effects of solar outbursts on terrestrial systems, predictions of these events are highly desirable. A step towards forecasting might be, apart from sunspot monitoring and far-side watch, the heliotomographic observation and diagnostics of emerging active regions (see Section 5.2).

Actual forecasting can only be effective if it uses a combination of comprehensive observations and models of the Sun and of the heliosphere, the Earth's magnetosphere, ionosphere and thermosphere. Large research efforts are currently being made in the development of corresponding models and their combination.

\subsection{The SUn AND THE EARTH's CLIMATE}

For a long time, the effect of the long-term variability of the Sun on the Earth's climate has been a controversial issue. Today, it is considered as most probably real. The terrestrial climate depends on a number of complex processes, many of which are yet barely understood. On large time scales of several millennia, it is correlated with changes in the Earth's orbital motions around the Sun.

Eddy (1976) investigated historical records of sightings of aurorae and of sunspot numbers, as well as records of ${ }^{14} \mathrm{C}$ in tree rings (Figure 17), and found that these past events and time series evidenced some correlation with earlier solar minima and at least one maximum of activity in the past 2000 years. These coincided with periods of climatic extremes - the little 


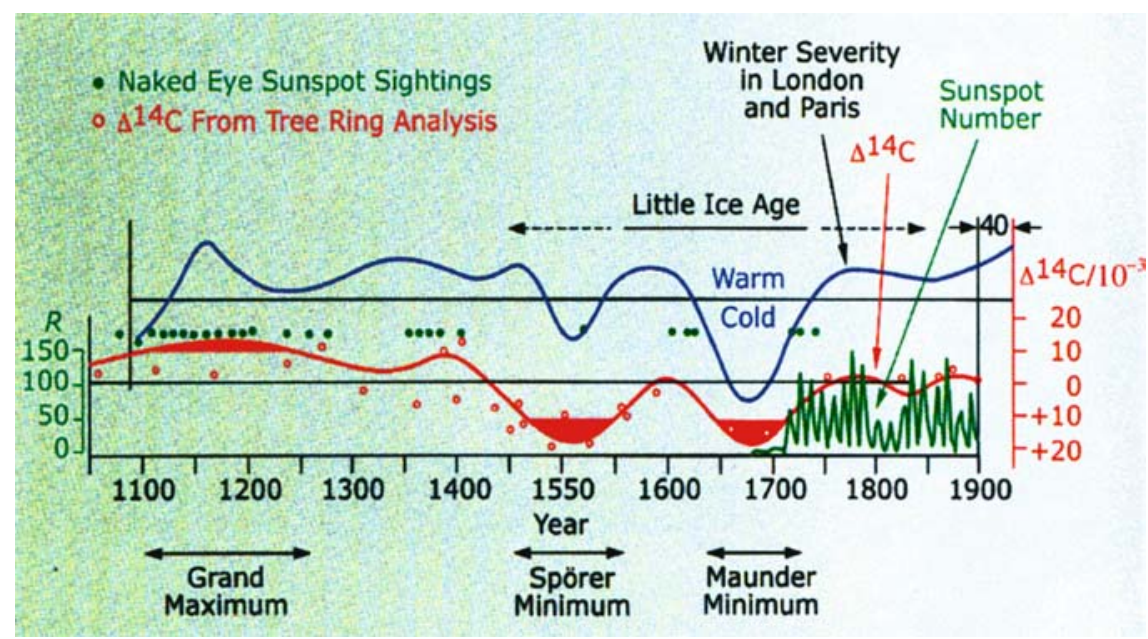

Figure 17. Time series of sunspot number R and ${ }^{14} \mathrm{C}$ measurements. From Eddy (1976).

ice age of the 16th to 19th century and the medieval climatic optimum of the eleventh through 13th centuries. He also noticed a complete absence of sunspots during a large fraction of the 17 th century, from 1645 to 1715 , as already noted by Spörer (1887) and Maunder (1890), and coined the expression the Maunder minimum.

During recent decades, global warming of $(0.6 \pm 0.2) \mathrm{K}$ has been observed on Earth (lntergovernmental Panel on Climate Change, IPCC, 2001). At the same time the amount of anthropogenic greenhouse gases (e.g., $\mathrm{CO}_{2}$ or methane) in the Earth's atmosphere has increased dramatically. It is thus of crucial importance to know the influence of the Sun on the evolution of the Earth's environmental parameters, in order to distinguish that from other influences, such as, e.g., volcanic eruptions and anthropogenic factors. A summary of the solar processes that might be important for the terrestrial climate is given by Hoyt and Schatten (1997) and in the book edited by Friis-Christensen et al. (2000).

\subsubsection{Total irradiance}

An obvious candidate for long-term trends is the changing solar activity causing variability in irradiance and thus of the temperature distribution in the Earth's atmosphere. Direct long-term observational time series of possible solar forcing parameters, e.g., of the irradiance, are not available before the late seventies of the last century, and suitable proxies have to be employed. Most models use proxies like sunspots and faculae, solar cycle length, geomagnetic activity and cosmic ray flux for the reconstruction of solar irradiance time series (e.g., Solanki and Fligge, 2000). The sunspot number is the most widely used such proxy, and the one recorded for the 
longest time. Figure 18 shows the record of sunspot numbers from 850 until today, from indirect reconstruction and direct observations (after 1650).

From a model reconstruction, Lean et al. (1995) estimated the irradiance level during the Maunder minimum to be $0.25 \%$ lower than today, which is not enough to explain the observed climate changes.

\subsubsection{UV irradiance}

Changes in the solar spectrum, especially in the UV, affect stratospheric temperature and chemistry (e.g., the balance between ozone production and destruction), and thus change the thermodynamical structure of the middle atmosphere, as has been investigated by Haigh (1996, 1999). Shortward of $102.6 \mathrm{~nm}$, the $\mathrm{O}_{2}$ photoionization limit, the EUV is responsible for the day-time ionosphere and its heating. EUV absorption also leads to other important processes in the Earth's atmosphere, including photoionization of $\mathrm{N}_{2}, \mathrm{O}$ and $\mathrm{NO}$ at wavelengths shorter than 79.6, 91.1, and $134.0 \mathrm{~nm}$, respectively, as well as photodissociation of $\mathrm{N}_{2}$ shortward of $127.0 \mathrm{~nm}$ and $\mathrm{O}_{2}$ shortward of $242.2 \mathrm{~nm}$. The latter process is the key step in ozone formation. Ozone destruction happens at somewhat larger wavelengths, e.g., in the Hartley band, shortward of $310 \mathrm{~nm}$ (Salby, 1996; Haigh, 2004). Solar variability is greater in the far UV range, and thus ozone

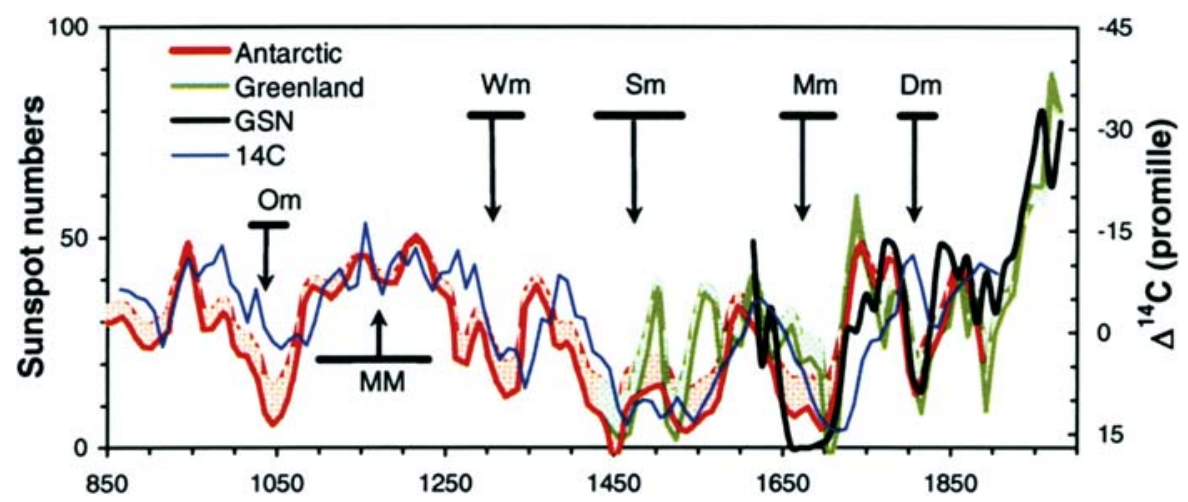

Figure 18. Time series of the sunspot number as reconstructed from ${ }^{10} \mathrm{Be}$ concentrations in ice cores from Antarctica (red) and Greenland (green). The corresponding profiles are bounded by the actual reconstruction results (upper envelope to the shaded areas) and by the reconstructed values corrected at low values of the sunspot number (solid curves) by taking into account the residual level of solar activity in the limit of vanishing sunspot number. The black curve shows the observed sunspot number since 1610 (group sunspot number, GSN) and the blue curve gives the ${ }^{14} \mathrm{C}$ concentration in tree rings. Several minima (Dalton, Maunder, Spörer, Wolf, Oort) and the medieval maximum of solar activity are also marked. (Reprinted from Usoskin et al., Phys. Rev. Lett., vol. 91, Issue 21, id. 21110, 2003, Copyright (2004) by the American Physical Society). 
production is more strongly modulated by solar activity than its destruction, the net production of stratospheric ozone being higher during periods of higher solar activity. The enhanced concentrations of stratospheric ozone result in greater absorption at wavelengths shorter than $330 \mathrm{~nm}$ and larger than $500 \mathrm{~nm}$, leading to less radiation reaching the troposphere at solar maximum. However, these effects are strongly non-linear, and the estimates of their impacts on the terrestrial climate system vary widely (Haigh, 2004). Like the total solar irradiance, the UV irradiance has been monitored since the advent of space research in the late seventies of the last century (Rottman et al., 2001).

\subsubsection{Cosmic rays}

A more indirect connection between solar activity and the Earth's climate has been proposed to act via cosmic rays. Cosmic ray fluxes are the main cause for the ionization of the atmosphere below 55 to $60 \mathrm{~km}$. Thus, cosmic rays could possibly result in the electrification of aerosols, increasing the latter's effectiveness to act as condensation nuclei. Marsh and Svensmark (2000) described a relation between the rate of formation of low altitude clouds and the flux of cosmic rays that might happen via the interaction with aerosols.

\subsubsection{Reconstruction of past climate and activity records}

During the past two decades much progress has been made in measuring detailed records of cosmogenic nuclides $\left({ }^{10} \mathrm{Be},{ }^{14} \mathrm{C}\right.$, and others) in various natural archives such as ice and sediment cores and tree rings. These records provide unique long-term information on the heliomagnetic and geomagnetic modulation of the cosmic rays and the transport and distribution of cosmogenic nuclides in the Earth system. The modulation of the cosmic ray flux by the solar wind (cf., Section 2.3.4) is the reason for ${ }^{14} \mathrm{C}$ variations in the tree-ring record over the past millennium (Stuiver, 1961). At times of greater solar activity the solar wind and its magnetic field shield the Earth from ${ }^{14} \mathrm{C}$-generating cosmic rays, resulting in lower ${ }^{14} \mathrm{C}$ absorption by living organisms. The same anticorrelation with solar activity holds true for the radioactive isotope ${ }^{10} \mathrm{Be}$ in precipitated snow, that can be accessed in ice cores from Arctic areas. These indirect indices have the potential to extend the records of solar variability significantly, to the past 100,000 years, using accurately dated ice cores (Beer, 2000).

\subsubsection{Present status of knowledge}

Lockwood et al. (1999) used measurements of the near-Earth magnetic field, namely the geomagnetic activity index, aa (from "auroral activity"), to reconstruct the heliospheric field, which is generated by the solar open magnetic flux dragged out by the solar wind. They found an increase of 
the open magnetic flux by approximately a factor of two during the last century. Mende and Stellmacher (2000) compared past temperature records of the Earth with proxies of solar variability $\left({ }^{10} \mathrm{Be}\right.$ from ice cores) and of anthropogenic influence (measurements of atmospheric greenhouse gases and from ice cores, in particular the $\mathrm{CO}_{2}$ record at Mauna Loa). A combination of estimated solar and anthropogenic functions was fitted to reproduce the observed temperature increase. For the time period from 1856 to 1996, they identified both an increasing anthropogenic forcing and an increasing solar influence, as the main driving forces for the Earth's climate, both being of roughly the same order of magnitude.

Recently, Usokin et al. (2003, 2004) used measurements of ${ }^{10} \mathrm{Be}$ to reconstruct via an inversion method a record of sunspot numbers dating back to the year 850 (Figure 18). They could extend the findings of Lockwood et al. (1999) and Mende and Stellmacher (2000) that the Sun is currently (since about 1940) in a phase of high level activity, not only relative to the last few centuries but also a millennium scale.

However, Solanki and Krivova (2003) estimated that the joint influence of solar variability through tropospheric heating by irradiance changes, changes in the UV spectrum or changes in the cosmic ray flux, could not account for more than $30 \%$ of the steep temperature increase observed during the last 30 years (Figure 19). Although there still remains the possibility of unrecognized processes, all this suggests that anthropogenic forcing and solar influences presently act in the same direction to cause global warming of the Earth.

\section{Future space observations}

As usual in science and particularly in astronomy, progress in observing techniques yields both expected and unexpected discoveries and, at the same time, raises new and yet unanswered questions. In the case of the Sun, we witness exactly that situation, particularly because of the unique character of the advanced missions discussed in this paper, and because we genuinely did explore the unknown.

The enormous knowledge accumulated over the past 15 years leaves several unanswered questions that can only be solved with more sophisticated observations. Despite the important progress witnessed in ground-based observations, the fact that they only cover the visible and radio part of the spectrum, plus a few infrared windows, clearly indicates that primarily future space missions will yield the required improvements. Several such missions are in development - or soon will be - either in the US, Europe, or Japan and in the rest of the world. A comprehensive description of these facilities can be found in Fleck (2002). In this Section, we mainly 


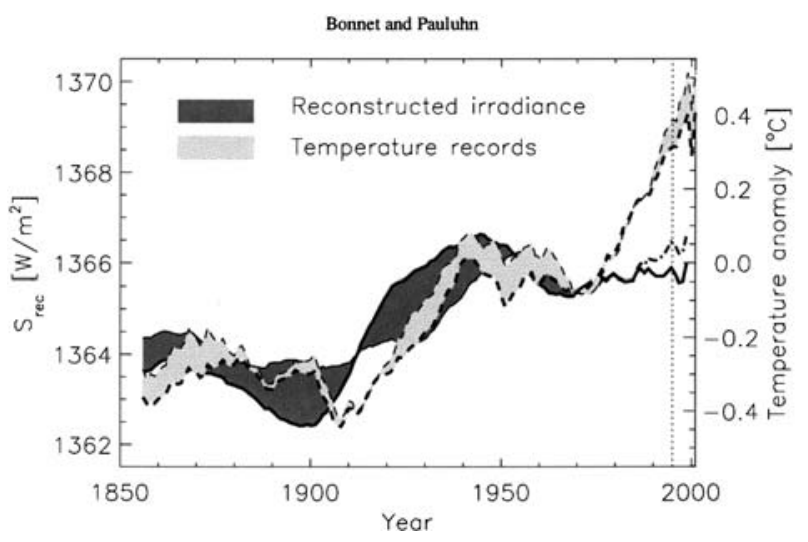

Figure 19. Total solar irradiance and terrestrial temperature vs. time for irradiance reconstructions with an increase in the 11-year averaged irradiance between 1700 and 1980 of $4 \mathrm{~W} / \mathrm{m}^{2}$. The dark shaded curves prior to 1985 represent irradiance reconstructions (solid curve: cycle-length based, dashed: cycle-amplitude based). From 1985 onward they represent total irradiance measurements. The light-shaded curves represent global (dark) and northern hemisphere (light) temperatures. All curves have been smoothed by an 11-year running mean. After the date marked by the vertical dotted line, the averaging period has been adjusted (Solanki and Krivova, 2003).

identify the remaining issues, without claiming to be exhaustive, outlining the required main characteristics of the corresponding future missions. This is undoubtedly a subjective approach. We do think, however, that the present literature as referred to in the previous Sections is abundant enough to satisfy the reader in the need of comprehensiveness.

\subsection{THE DEEP SOLAR INTERIOR}

Our present knowledge of the deep interior is not fully compatible with the trend of physical parameters as deduced by helioseismology. More work is necessary in adjusting the solar model by introducing more ${ }^{3} \mathrm{He}$ mixing in particular. Furthermore, we do not yet reach the deep core of the interior below a few percent of the solar radius, although we do not anticipate a dramatic difference with the presently deduced trend of density and temperature.

Further improvements in the measurements require access to the g-modes that pervade the whole body of the Sun from the very centre to the surface. Appourchaux (2003) does not leave too many hopes that we can access g-modes in the average lifetime of a solar astronomer with the present techniques, due to the necessity of integrating measurements for several decades in order to reach the estimated required accuracy of a fraction of $1 \mathrm{~mm} / \mathrm{s}$ in radial velocity measurements. Fortunately, new techniques are being envisaged that should yield substantial improvements. 
7.1.1. Measuring radial velocities down to $0.1 \mathrm{~mm} / \mathrm{s}$

The limit of around $3 \mathrm{~mm} / \mathrm{s}$ presently achieved is indeed insufficient for the detection of g-modes. Longer observing times or higher sensitivities are required above the present capabilities of the GOLF (Global Oscillations at Low Frequencies, Gabriel et al., 1995) instrument on SOHO or of the BiSON network, even though, using improved algorithms, velocity signals down to $2 \mathrm{~mm} / \mathrm{s}$ have been detected in long time series from GOLF (Turck-Chièze et al., 2004). A successor of GOLF, the New Generation GOLF or NGGOLF is being proposed for future space missions, which would derive radial velocities through 15 points in the wing of the sodium $\mathrm{D}$ line (Turck-Chièze et al., $2001 \mathrm{~b}$ ), with an expected accuracy of $0.1 \mathrm{~mm} / \mathrm{s}$, solar noise permitting. In parallel, at Nice University, a Mach-Zender interferometer is being developed (Jacob, 2002). Its robust and compact nature, similar to that of the Michelson Doppler Interferometer on SOHO, with no moving parts, makes it particularly suited to space-borne measurements. Its performance would be similar to that of NGGOLF.

\subsubsection{Solar limb oscillations measurements}

Using intensity fluctuations, Appourchaux and Toutain (1998) and Toner et al. (1999) showed that p-modes could be detected in the limb data of MDI and were amplified relative to full disk measurements by a factor of 4 to 5 . This observed amplification of the modes could be used to attempt to detect g-modes with an instrument measuring the fluctuations of the limb at the $10^{-6}$-arcsec level and the solar irradiance with high precision, such as the PICARD micro-satellite of the French space agency CNES (Damé et al., 1999). A similar approach is used in the Helioseismic and Magnetic Imager (HMI) (http://hmi.stanford.edu) of Stanford University foreseen to fly on board NASA's Solar Dynamics Observatory (SDO).

\subsubsection{Detection of gravitational waves}

A more revolutionary approach would be to detect the tidal perturbations of the gravitational field produced by the g-modes of order $\geq 2$. Such measurements might be possible with the ESA/NASA Laser Interferometer Space Antenna (LISA) to be launched around 2012, with a detection level equivalent to a few $\mathrm{mm} / \mathrm{s}$, probably at the limit of clear detection. A more sensitive mission, ASTROD (http://gravity5.phys.nthu.edu.tw/ ASTROD), using laser ranging of two satellites located on the far side of the Sun in the Earth's orbit, unfortunately not approved yet, would reach beyond the theoretical amplitude of the g-modes (Kumar et al., 1996), and appears today as the ultimate concept. Figure 20 (from Appourchaux, 2003) presents a summary of the limits reached by the various techniques described in this paragraph. 


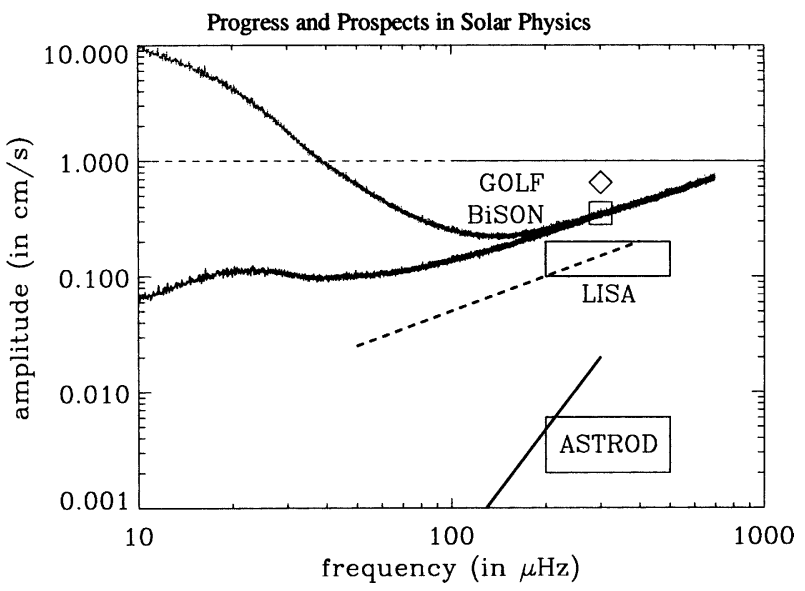

Figure 20. Predicted upper detection limit of gravitational modes for various existing and planned missions. (From Appourchaux, 2003).

\subsection{SOLAR INTERNAL DYNAMICS AND THE GENERATION OF THE SUN'S MAGNETIC FIELD}

\subsubsection{Local helioseismology}

Mode splitting helioseismology observations, together with three-dimensional modelling of convection coupled to rotation, have provided a fairly detailed knowledge of the dynamics of the solar interior. Puzzling, however, is the rotation of the highly turbulent convection zone around the tachocline and below the solar surface. Modelling the transport of angular momentum in the interior requires a better knowledge of the processes which couple the radiatively dominated core to the convective envelope (Thompson et al., 2003). The situation appears even more complex after the discovery of the roughly periodic 1.3 years variations in the equatorial region near the tachocline. At higher latitudes, similar variations are observed with less marked amplitudes and a period of 1 year. These results are at the limit of statistics and need to be confirmed. Because it is assumed that the source of the solar magnetic field is a global dynamo mechanism located at the tachocline, these discoveries, indicative of dynamical changes close to or within the site of the dynamo, cast new light on the processes through which the magnetic field is generated, and may provide important clues on its perennial operations. The whole mechanism of the magnetic cycle might consequently be better understood, and its variations over longer periods of time find an explanation, potentially leading to predicting its characteristics, in particular its amplitude and its periodicity, and possibly allowing forecasts of solar activity. Observing internal motions and rotation, both over long and short time scales through local helioseismology techniques, and modelling through 3-D computation pro- 
grams, are the essential tools to better understand the complexities of the interplay between convection, magnetic buoyancy and flows that create the characteristic manifestation of the magnetic cycle. Developing these tools will constitute important progress.

The magnetic field is transported upward through the convective zone to the photosphere and the corona by magnetic buoyancy, and this process is affected by solar flows. These may be the source of the explosive events that are observed higher up in the atmosphere, such as flares and CMEs. Similarly, the shears in velocity fields below the photosphere result in the shearing and twisting of the field lines that should add energy to the explosive release of the field (Toomre, 2003).

Following SOHO and its successful set of instruments, the next step in local helioseismology (besides the refinement of the instrumentation of ground based networks) will be NASA's Solar Dynamics Observatory (SDO) (http://sdo.gsfc.nasa.gov/) and in particular its Helioseismic and Magnetic Imager, a modern version of SOHO's MDI instrument. The HMI will provide continuous full disk 1-arcsec resolution oscillation measurements and magnetograms at an interval of less than $50 \mathrm{~s}$. SDO will be launched in 2008 into a geosynchronous orbit.

\subsubsection{Observation of the solar poles}

Because of the importance of the latitudinal variations of rotation and of the thermal structure in the convection zone for understanding the generation and transport of the magnetic field through the cycle, it is imperative to improve the accuracy of measurements at high latitudes. The high-latitude results are based essentially on observations of high-degree modes $(l \geq 250)$ whose reliability is questionable near the poles (Howe, 2003). All helioseismology observations have up to now been conducted from the ecliptic plane, be it from the ground or from space. The only mission which could observe the poles, Ulysses, was deprived of its imaging capability, when NASA decided to abandon the second satellite of the original Out-of-Ecliptic mission (Figure 21). ${ }^{1}$

ESA's Solar Orbiter, (http://www.rssd.esa.int/SH-general/Projects/Solarorbiter/), which is able to reach latitudes some $38^{\circ}$ above the ecliptic plane at a distance of $45 \mathrm{R}_{\odot}$, with a record equivalent resolution of $0.05^{\prime \prime}$ from Earth corresponding to $35 \mathrm{~km}$ per pixel, would go a long way to improving the present situation. This may still be limited for probing the highest latitudes. An ideal mission would be a repeat of the original Out-of-Ecliptic Mission. In this new proposed twin-spacecraft out-of-ecliptic mission,

\footnotetext{
${ }^{1}$ The OOE mission was orginally composed of two symmetrical spacecraft in solar oppsition on their common orbit perpendicular to the ecliptic, so as to observe each pole simultaneously.
} 


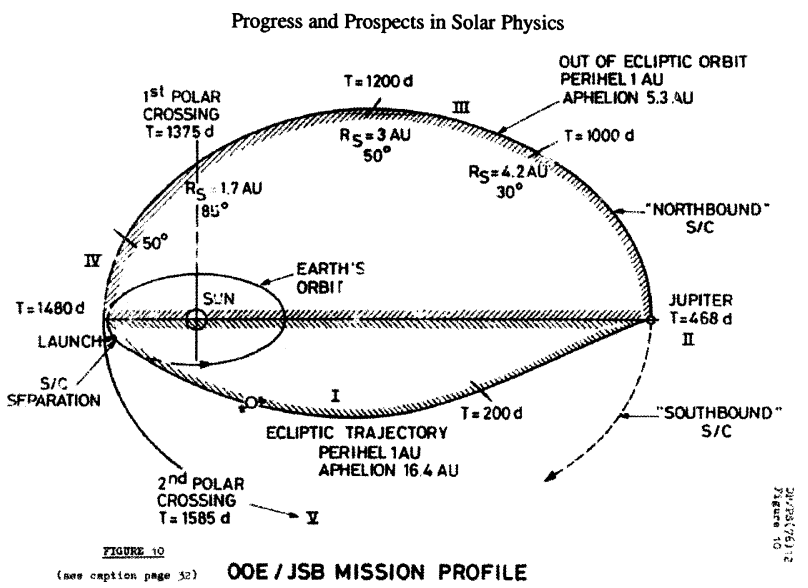

Figure 21. Concept of the twin satellite out-of-ecliptic mission, from the original concept of Ulysses.

each spacecraft should be equipped with exactly the same set of instruments, in particular the most modern version of the SDO HMI. The simultaneity of observations would add a tremendous power to the study of the solar interior with helioseismology. It would also provide a unique tool for forecasting solar activity by being able to observe both sides of the Sun at the same time.

\subsection{THE HOT EXPANDING MAGNETIC SOLAR ATMOSPHERE}

The magnetic field generated by the solar dynamo plays the most predominant role in shaping the solar atmosphere from scales as large as the chromospheric network down to spicules and chromospheric bright grains (Peter, 2002). It determines its temperature, its dynamics and its variability. It is the support of the chromosphere and of the corona and the cause of the solar wind. Ultimately, it is the element that makes our star active, and that influences the interplanetary environment and the planets of the solar system. Future missions must therefore target studies of the magnetic field for understanding the behaviour and the exact role of this most important element of the Sun's extended atmosphere. However, the techniques for measuring the field intensity either in the chromosphere or in the corona are rather limited.

The method using the Hanlé effect (Stenflo et al., 1998; Raouafi et al., 2002) is a promising one but its implementation is still raising technical problems. A valuable diagnostic of coronal magnetic fields is radio emission, and coronal magnetography one of the aims of the proposed Frequency Agile Solar Radiotelescope (http://www.ovsa.njit.edu/fasr) (FASR, see, e.g., White et al., 2003). 
7.3.1. Higher resolution and three-dimensional observations for an improved understanding of the heating mechanisms

Although the unique set of instruments carried on board SOHO has provided a three-dimensional understanding of the solar atmosphere and has revealed, together with Yohkoh and TRACE, the magnetic nature of the heating mechanisms, there are still a number of unanswered questions. The role of frequent magnetic reconnections associated with the cancellation and disappearance of numerous small magnetic bipolar regions has been shown to be predominant in the heating process, but how much of the energy is provided by the small-scale reconnection events and the associated flaring processes is not yet clear. The power of SOHO to make discoveries comes mainly from the uninterrupted character of its observations. To go further in the comprehension of these small-scale processes, one must have access to higher-resolution and three-dimensional observations. Two new satellites carry great promise in these respects and would improve substantially the present status.

SOLAR-B is a Japanese mission with US and UK participation. It is a successor to Yohkoh, planned to be launched in 2006. For a more complete description see Fleck (2002), and the website of the Japanese space agency (JAXA) (http://www.jaxa.jp/missions/projects/sat/astronomy/ solar_b/index_e.html). Its scientific payload comprises in particular a diffraction limited $50 \mathrm{~cm}$ telescope, capable of sub-arcsec resolution $\left(\leq 0.1^{\prime \prime}\right)$ in the spectral range $388-660 \mathrm{~nm}$. Its grazing incidence X-ray telescope would provide full Sun coronal images at wavelengths from 0.2 to $6 \mathrm{~nm}$ with $1^{\prime \prime}$ resolution. SDO, already mentioned, will also strongly contribute to this domain.

Also aiming at high resolution (35 $\mathrm{km}$ on the Sun) is SUNRISE (http:// www.linmpi.mpg.de/english/projekte/sunrise/), a balloon-borne 1-m solar telescope that is coordinated by the Max-Planck-Institut für Sonnensystem-forsrung (MPSe, Germany) with international collaboration. Beginning in $2005 / 2006$, the telescope will be operated during a series of stratospheric balloon flights in Antarctica.

The Space Solar Telescope (SST)(http://ilws.gsfc.nasa.gov/space-solartelescope.pdf), another high-resolution mission, is being developed by the National Astronomical Observatories of the Chinese Academy of Sciences (NAOC). It is foreseen to be launched in 2005 into a Sun-synchronous orbit at a height of $735 \mathrm{~km}$ and an inclination of $98.3^{\circ}$.

It is likely, however, that super-high resolution might be necessary to understand fully the small-scale processes. This could be the domain of projects like SIMURIS (Damé, 1998), or the Reconnection and Microscale Probe, RAM, a candidate mission in NASA's Sun Earth Connection roadmap (Fleck, 2002). With expected performance of a few 0.001" and high time resolution, these projects may address the fundamental processes at 
play in magnetic reconnection, microscale instabilities and particle acceleration. However, the capability of accessing super high resolution observations with enough light flux is still to be demonstrated.

\subsubsection{In situ and stereoscopic observations to further understand the solar wind}

SOHO and Ulysses, as well as Yohkoh, have yielded major progress in our understanding of the solar wind: its slow and fast components, its composition, its sources, its acceleration and its major physical properties. The unique orbit of Ulysses above the ecliptic plane, and the high resolution coronagraphs and spectrometers of SOHO have been key elements for these achievements. Because they have been observing the Sun over solar minimum and maximum we know better how the solar wind's properties vary over the solar cycle.

It is now clear that the acceleration of the fast wind takes place within $10 R_{\odot}$ of the surface, while the slow wind is accelerated over much larger distances, reaching terminal velocities around 20 to $30 R_{\odot}$. Further progress will require refined measurements. Solar physicists envisage two approaches: (1) observations of structures and velocity fields through imagery and spectrometry, and (2) in situ measurements at close distances to the Sun.

In the first category, it is expected to extend the UVCS type measurements over several spectral lines such as $\mathrm{He}$ I Ly $\beta$, the $\mathrm{O}$ IV doublet at $103.0 \mathrm{~nm}$ or the He II $30.4 \mathrm{~nm}$ resonance line. Because the light flux from these lines is rather weak compared with that for the resonance line of hydrogen, the sensitivity of the respective instruments will have to be at least one order of magnitude higher. Advanced coronagraphs of the LASCO type capable of observing closer to the Sun $\left(1.1 R_{\odot}\right)$ and with polarimetry capabilities will help to observe the acceleration processes at the very base of the corona. This is the objective of the Advanced Spectroscopic and Coronographic Explorer (ASCE) of NASA (Fleck, 2002). ASCE would be placed in an orbit equivalent to that of the Hubble Space Telescope, i.e., $600 \mathrm{~km}$ altitude and $28.5^{\circ}$ inclination. In this area also, the already mentioned twin-satellites of the Out-of-Ecliptic mission would prove to be invaluable tools in the understanding of the fast component of the solar wind. Similarly, ESA's Solar Orbiter will analyze the slow component.

Much more demanding and certainly at the limit of today's technological capabilities of NASA and ESA is the in situ Solar Probe (http://solarprobe.gsfc.nasa.gov/solarprobe_mission.htm, Habbal et al., 1998). The concept has been studied for more than 30 years and has still not yet materialized! Obviously, the thermal environment very close to the Sun with a perihelion at $4 R_{\odot}\left(3 R_{\odot}\right.$ above the surface) is one of the main technologi- 
cal difficulties to overcome when building such an ambitious and revolutionary mission. The proposed orbit is extremely interesting for the study of the acceleration regions of both the fast and slow solar wind. The payload would incorporate both in situ and remote sensing instruments: a magnetometer, spectrometers to measure solar wind, electrons, and energetic particle composition, X-ray telescopes, coronagraphs, etc. Due to the rapidity of the probe's transit into the corona, such local measurements need to be complemented by observations and more continuous sets of data such as those permitted by the Solar Orbiter, in order to reconstruct the structures and the environment in which the acceleration and heating processes take place. Because of the importance of the heliospheric latitude dependence of the acceleration mechanisms, the Solar Probe should be placed in a polar orbit.

\subsection{Connecting the Sun AND the Earth}

Pursuing the study of the Sun is clearly an objective of fundamental astrophysical importance. At the same time, the solar influence on the interplanetary medium and on the Earth is a matter of growing importance, as has been discussed in Section 6. Hence there is a need to monitor continuously the solar particle and radiative emissions, as well as their short and long term evolution; this is critical for eventually forecasting space weather.

Recent discussions at the international level between NASA, ESA, JAXA and ROSAVIACOSMOS have led to the proposal of the internationalisation of NASA's original Living with a Star (LWS) programme. Such an in international framework, called International Living With a Star (ILWS) (http://ilws.gsfc.nasa.gov), would coordinate all solar-physics missions of these respective agencies, and possibly others, in a most comprehensive way. ILWS will include missions still in operation, such as Ulysses and SOHO (both extended until at least 2007), plus already approved and future missions in development or under study. In addition to the solar missions proper, an additional set of satellites particularly suited to the study of the interplanetary medium will form the heart of the ILWS (Figure 22).

The nucleus of this system will be the Solar Terrestrial Relation Observatory (STEREO) (http://stp.gsfc.nasa.gov/missions/stereo/stereo.htm), a NASA mission to be launched in 2005. STEREO will use two identical satellites with exactly the same payload, one drifting ahead of the Earth, the other one behind, providing a stereoscopic view of the coronal structures and of the CMEs, as well as of their propagation throughout the heliosphere and their effects at Earth.

Solar Sentinels (http://lws.gsfc.nasa.gov/sentinels.htm) are the heliospheric element of the LWS programme to discover, understand and model 
Present Solar-Terrestrial Missions \& "First Order" ILWS Missions

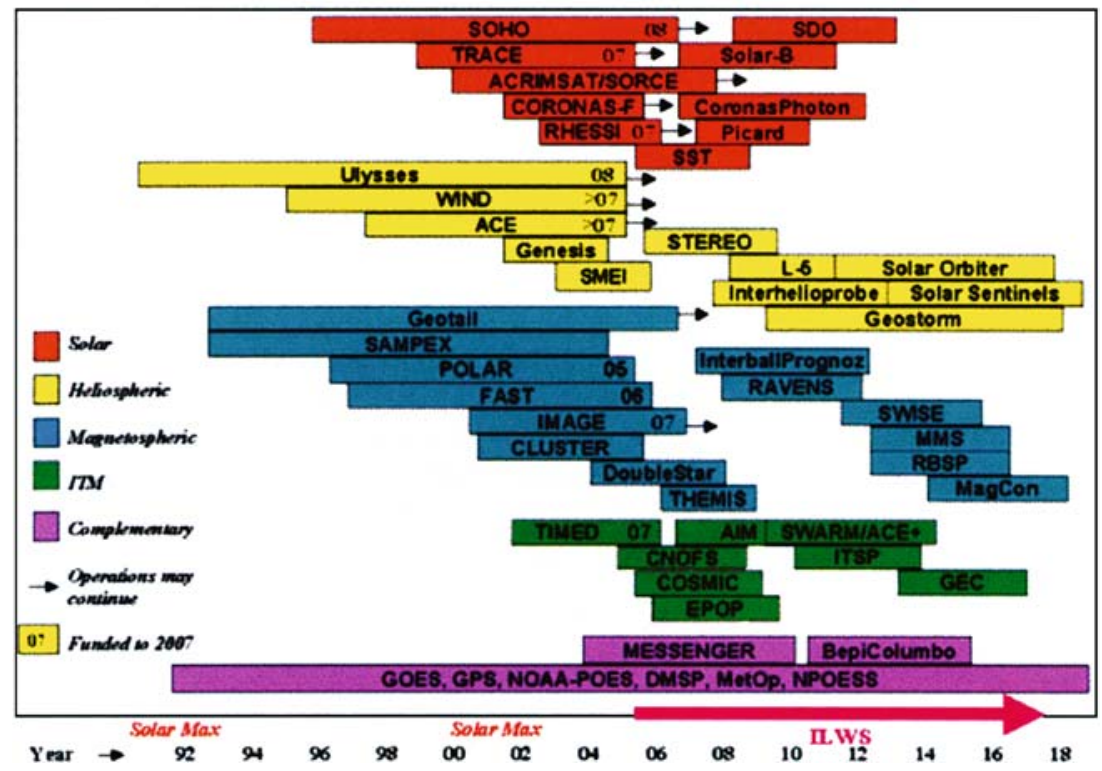

Figure 22. Present Solar- Terrestestrial Missions and First Order ILWS Missions. The figure also identifies some of the international solar-terrestrial missions currently under development or in planning that are associated with the ILWS program. Credit: NASA.

the connection between solar phenomena and geospace disturbances. They form a set of three small spacecraft plus a larger one, flying in different highly eccentric orbits in the inner heliosphere. They will be equipped with in situ experiments. These multi-point measurements will be used to determine the evolution and propagation of solar wind/solar transients as they propagate from the Sun to the Earth and will study the generation of shocks and associated energetic particles. At the same time, the Geospace Storm Probes (http://lws.gsfc.nasa.gov/geospace.htm) of NASA will monitor the near Earth space plasma current systems. The joint operation of these probes and of the SDO will allow the study of solar forcing the Earth's upper atmosphere, and the effects of solar activity on the magnetosphere and the Van Allen radiation belts.

\section{Conclusion}

In the last 20 years, many observations have been made on the Sun, both from the ground and from space. This has been mainly motivated by the fact that our star is a unique astrophysical laboratory that we can study in sufficient detail in order to test our theories of stellar interiors and of stel- 
lar atmospheres. In recent years, we have also witnessed a growing interest in the field of solar terrestrial relations in order to understand better the influence of the Sun on the status and on the evolution of our planet.

Tremendous progress has been accomplished in all fields, from the study of the solar interior, the role of the solar magnetic field, the heating of the chromosphere and of the corona to the sources and acceleration mechanisms of the solar wind and the perturbations of the heliosphere, in particular those caused by Coronal Mass Ejections (CMEs). These advances are due to the increased performance of ground-based instrumentation, but to a greater extent to the availability of a set of unique space missions, such as Yohkoh, SOHO, TRACE and Ulysses.

Despite the availability of these powerful tools, several key questions still need to be analysed in greater detail. This is the case for the generation mechanisms of the magnetic field in the convective zone, and the role of differential rotation and of its interplay with convection in creating the dynamo effect. Even if the role of the magnetic field in the coronal heating process and in the acceleration of the solar wind is now clearly evidenced, there are still a large number of problems to be solved before we can really understand the details and small-scale processes at play. The role of magnetic reconnection seems to be important also in the manifestation of CMEs, but we are far from being in a position to forecast them accurately.

This clearly shows that new instruments and new observations are needed. Higher spatial resolution will provide better insight into the smallscale processes at play, in particular in the heating of the corona and solar wind acceleration. Stereoscopic observations are crucial to understand the larger-scale processes which affect the changes in the configuration of the magnetic field and result in the CME phenomenon. Up to now, the Sun has been observed at all wavelength ranges only from the ecliptic plane. This is limiting our understanding of the processes which occur at the Sun's North and South poles. In particular the accuracy of the measurements of solar differential rotation in that crucial polar zone is very poor. This illustrates the need for a set of missions to be designed to observe the poles from above the ecliptic plane, if possible simultaneously from both hemispheres, equipped with visible and EUV telescopes, magnetometers and coronagraphs. Finally, it is clear that in situ measurements will prove invaluable in analysing and understanding the processes at play in the corona. In that respect, the long awaited Solar Probe would provide the unique set of observations that should cast new light on the physics of the extended atmosphere of the Sun.

These observations become more and more necessary if we are to forecast solar influences on our planet, and the possible disturbances induced by the Sun on the Earth's climate and on the systems that characterise our modern civilization. The remaining problems in both solar physics and in 
the new discipline of space weather forecasting require a large number of missions operated in a coordinated way. The International Living With a Star programme, which is an internationalisation of NASA's LWS programme, appears to be one of the most promising tools in this respect. It assembles in a unique system the missions which are in development or foreseen in the future by a large number of space faring nations or agencies. International cooperation therefore will be an absolute necessity in attempting to better understand and evaluate both the positive and negative effects of our Sun on our daily life.

\section{Acknowledgements}

We would like to thank all colleagues who have generously provided material and advice. In particular we want to express our gratitude to Yasmine Calisesi, Claus Fröhlich, Johannes Geiss, Costis Gontikakis, Alexander Kosovichev, Natalie Krivova, Dave McComas, Pascal Saint-Hilaire, Sami Solanki, Rudolf von Steiger, Michael Thompson, Ilya Usoskin, Klaus Wilhelm, and many more, especially those maintaining the websites of the various missions. The editor, Michael J. Rycroft, and the reviewers are thanked for helpful remarks and comments which significantly improved the content and readability of the paper.

\section{References}

Alexander, D. and Acton, L. W.: 2001, 'The Active Sun', in J. Bleeker, J. Geiss and M.C.E. Huber (eds.), The Century of Space Science, KIuwer Academic Publishers, Dordrecht, The Netherlands, 1089-1113.

Alfvén, H.: 1947, 'Granulation, Magneto-Hydrodynamic Waves, and the Heating of the Solar Corona', Man. Not. Roy. Astronom. Soc. 107, 211-219.

Antiochos, S. K.: 1979, 'The Stability of Solar Coronal Loops', Astrophys. J. Lett. 232, LI25-L129.

Appourchaux, T.: 2003, 'The Search for g-Modes', ESA SP-517, ed.: H. Sawaya-Lacoste, ESA Publications Division, Noordwijk, The Netherlands, 131-138.

Appourchaux, T. and Toutain, T.: 1998, in J. Provost and F.-X. Schmider (eds.), Sounding Solar and Stellar Interiors, IAU Symp. 181, Poster volume, 5, Kluwer Academic Publishers, Dordrecht, The Netherlands.

Asai, A., Shimojo, M., Isobe, H., et al.: 2001, 'Periodic Acceleration of Electrons in the 1998. November 10 Solar Flare', Astrophys. J. 562, L103-L106.

Aschwanden, M. J., Fletcher, L., Schrijver, C. J., and Alexander, D.: 1999, 'Coronal Loop Oscillators Observed with the Transition Region and Coronal Explorer', Astrophys. $J$. 520, 880-894.

Aschwanden, M. J.: 2001, 'An Evaluation of Coronal Heating Models for Active Regions. Based on Yohkoh, SOBO, and TRACE Observations', Astrophys. J. 560, 1035-1044.

Aschwanden, M. J.: 2002, 'Observation and Models of Coronal Loops: from Yohkoh to TRACE', ESA SP-505, ed.: H. Sawaya-Lacoste, Noordwijk, The Netherlands, ESA Publications Division, 191-198. 
Aschwanden, M. J. and Pernell, C. E.: 2002, 'Nanoflare Statistics from First Principles: Fractal Geometry and Temperature Synthesis', Astrophys. J. 572, 1048-1071.

Aschwanden, M.J., De Pontieu, B., Schrijver, C. J., and Title, AM.: 2002, 'Transverse Oscillations in Coronal Loops Observed with TRACE - II. Measurements of Geometric and Physical Parameters', Solar Phys. 206, 99-132.

Aschwanden, M. J., Nakariakov, V. M., and Melnikov, V. F.: 2004, 'Magnetohydrodynamic Sausage-Mode Oscillations in Coronal Loops', Astrophys. J. 600, 458-463.

Athay, RG.: 1966, 'Theoretical Line Intensities. V. Solar UV Emission Lines of Heavy Elements', Astrophys. J. 145, 748-795.

Athay, R G., White, O. R.: 1978, 'Chromospheric and Coronal Heating by Sound Waves', Astrophys. J. 226, 1135-1139.

Ayres, T.R., Testerman, L., and Brault, J. W.: 1986, 'Fourier Transform Spectrometer Observations of Solar Carbon Monoxide. II - Simultaneous Cospatial Measurements of the Fundamental and First-Overtone Bands, and CaII K, in Quiet and Active Regions', Astrophys. J. 304, 542-559.

Babcock, H. W.: 1953, 'The Solar Magnetograph', Astrophys. J. 118, 387-396.

Babcock, H. W. and Babcock, H.D.: 1955,'The Sun's Magnetic Field, 1952-1954'. Astrophys. J. 121, 349-366.

Babcock, H. W.: 1961, 'The Topology of the Sun's Magnetic Field and the 22-Year Cycle', Astrophys. J. 133, 572-587.

Bahcall, J. N. and Davis, R. Jr.: 1976, 'Solar Neutrinos: a Scientific Puzzle', Science 191, 264-267.

Bahcall, J. N., Pinsonneault, M. H., and Wasserburg, G. J.: 1995, 'Solar Models with Helium and Heavy-Element Diffusion', Reviews of Modem Physics 67, 781-808.

Banaszkiewicz, M., Witte, M., Rosenbauer, H.: 1996, 'Determination of Interstellar Helium Parameters from the ULYSSES-NEUTRAL GAS Experiment: Method of Data Analysis', Astron. Astrophys. Suppl. 120, 587-602.

Bastian, T. S., Benz, A. O., and Gary, D. E.: 1998, 'Radio Emission from Solar Flares', Ann. Rev. Astronom. Aslrophys. 36, 131-188.

Beck, J. G., Duvall, T. S., and Scherrer, P.H.:1998, 'Long-Lived Giant Cells Detected at the Surface of the Sun', Nature 394, 653-655.

Beer, J., Bilnov, A. Bonani, G., Finkel, R. C., Hofmann, H. J., et al. 'Use'of Be-10 in Polar Ice to Trace the 11-Year Cycle of Solar Activity', Nature 347, 1990, 164-166.

Beer, J., Tobias, S., Weiss, N.: 1998, 'An Active Sun Throughout the Maunder Minimum', Solar Phys. 181, 237-249.

Beer, J.: 2000, 'Long-Term Indirect Indices of Solar Variability', Space Sci. Rev. 94, 53-66.

Benz, A. O. and Krucker, S.: 2002, 'Energy Distribution of Microevents in the Quiet Solar Corona', Astrophys. J. 568, 413-421.

Bethe, H. A. and Critchfield, C. L.: 1938, 'The Formation of Deuterons by Proton Combination', Phys. Rev. 54, 248-254.

Bethe, H. A.: 1939, 'Energy Production in Stars', Phys. Rev. 55, 434-456.

Biermann, L.: 1941, Vierteljahrschrift Astronom. Ges. 76, 194.

Biermann, L.: 1946, Naturwissenschaften 33, 118.

Biermann, L.: 1948, 'Über die Ursache der Chromosphärischen Turbulenz und des UV Exzesses der Sonnenstrahlung', Zeitschrift fUr Astrophys. 25, 161-177.

Bohlin, J. D.: 1977, 'Extreme-Ultraviolet Observations of Coronal Holes. I - Locations, Sizes and Evolution of Coronal Holes, June 1973-January 1974', Solar Phys. 51, 377-398.

Bohlin, J. D. and Sheeley, N. R.: 1978, 'Extreme-Ultraviolet Observations of Coronal Holes.II - Association of Holes with Solar Magnetic Fields and a Model for their Formation during the Solar Cycle', Solar Phys. 56, 125-151. 
Bonnet, R. M.: 1968, 'Recherches sur l'emission Continue du Soleil entre $1950 \AA$ et 3000 A’, Thesis, Paris.

Bonnet, R. M., Decaudin, M., Bruner, E. C., Jr., et al.: 1980, 'High-Resolution Lymanalpha Filtergrams of the Sun', Astrophys. J. Lett. 237, L47-L50.

Bonnet, R. M., Decaudin, M., Foing, B., et al.: 1982, 'Rocket Photographs of Fine Structure and Wave Patterns in the Solar Temperature Minimum', Astronom. Astrophys. 111, 125-129.

Brekke, P., Thompson, W. T., Woods, T. N., and Eparvier, E. G.: 2000, 'The ExtremeUltraviolet Solar Irradiance Spectrum Observed with the Coronal Diagnostic Spectrometer (CDS) on SOHO', Astrophys. J. 536, 959-970.

Brković, A., Rüedi, I., Solanki, S. K., et al.: 2000, 'Characteristics Of Blinkers Observed With CDS', Astronom. Astrophys. 353, 1083-1093.

Brown, T.M.: 1985, 'Solar Rotation as a Function of Depth and Latitude', Nature 317, 591-594.

Brown, T. M. and Gilman, P.A.: 1984, 'Techniques for Detecting Giant Cells using Spatially Resolved Solar Velocity Data', Astrophys. J. 286, 804-809.

Brown, T. M. and Morrow, C. A.: 1987, 'Depth and Latitude Dependence of Solar Rotation', Astrophys. J. Lett. 314, L21-L26.

Brown, T. M., Christensen-Dalsgaard, J., Dziembowski, W. A., et al.: 1989, 'Inferring the Sun's Internal Angular Velocity from Observed p-Mode Frequency Splittings', Astrophys. J. 343, 526-546.

Brueckner, G. E. and Bartoe, J. -D. E.: 1978, "High Velocity Jets in the "Quiet" Sun as a Possible Source of the Solar Wind and the Heating of the Corona', Bull. Am. Astronom. Soc. 10, 416.

Brueckner, G. E., Howard. R. A., Koomen, M. J., et al.: 1995, 'The Large Angle Spectroscopic Coronagraph (LASCO)', Solar Phys. 162, 357-402.

Brun, A. S., Turck-Chièze, S., and Morel, P.: 1998, 'Standard Solar Models in the Light of New Helioseismic Constraints I. The Solar Core' Astrophys. J. 506, 913-923.

Brun, A. S., Turck-Chièze, S., and Zahn, J. P.: 1999, 'Standard Solar Models in the Light of New Helioseismic Constraints II. Mixing below the Convective Zone', Astrophys. J. 525, 1032-1041.

Bruner, E. C., Jr.: 1981, 'OSO 8 Observational Limits to the Acoustic Coronal Heating Mechanism', Astrophys. J. 247, 317-324.

Burlaga, L., Sittler, E., Mariani, E., and Schwenn, R.: 1981, 'Magnetic Loop behind an Interplanetary Shock - Voyager, Helios, and IMP 8 Observations', J. Geophys. Res. 86, 6673-6684.

Burnight, T. R.: 1949, 'Soft X-Radiation in the Upper Atmosphere', Phys. Rev. 76, 165.

Carlsson, M. and Stein, R. F.: 1995, 'Does a Nonmagnetic Solar Chromosphere Exist?', Astrophys. J. 440, L29-L32.

Carrington, R. C.: 1859, 'Description of a Singular Appearance Seen in the Sun on September 1, 1859', Mon. Not. Roy. Astronom. Soc. 20, 13-16.

Chaplin, W. J., Elsworth, Y., Isaak, G. R, et al.: 1996, 'Observing the Sun with the Birmingham Solar-Oscillations Network (BISON)', The Observatory 116, 32-33.

Chaplin, W. J., Christensen-Dalsgaard, J., Elsworth, Y., et al.: 1999, 'Rotation of the solar core from BISON and LOWL frequency observations', Mon. Not. Roy. Astronom. Soc. 308, 405-414.

Chapman, S. and Ferraro, V.C.A.: 1931, 'A New.Theory of Magnetic Storms', Terrestrial Magnetism and Atmospheric Electricity 36, 77-97.

Chou, D.-Y., Labonte, B. J., Braun, D. C., and Duvall, T. L., Jr,: 1991, 'Power Spectra of Solar Convection', Astrophys. J. 372, 314-320. 
Choudhuri, A. R.: 2003, 'The Solar Dynamo as a Model of the Solar Cycle', in B. N. Dwivedi (ed.), Dynamic Sun, Cambridge University Press, 103-127.

Craig, I. J. D., McClymont, A. N., and Underwood, J. H.: 1978, 'The Temperature and Density Structure of Active Region Coronal Loops', Astronom. Astrophys. 70, 1-11.

Cranmer, S. R, Field, G B., and Kohl, J. L.: 1999, 'Spectroscopic Constraints on Models of Ion Cyclotron Resonance Heating in the Polar Solar Corona and High-Speed Solar Wind', Astrophys. J. 518, 937-947.

Cranmer, S. R: 2000, 'Ion Cyclotron Wave Dissipation in the Solar Corona: The Summed Effect of More Thar 2000 Ion Species', Astrophys. J. 532, 1197-1208.

Christensen-Dalsgaard, J. and Gough, D.O.: 1981, 'Comparison of Observed Solar Wholedisk Oscillation Frequencies with the Predictions of a Sequence of Solar Models', Astronom. Astrophys. 104, 173-176.

Christensen-Dalsgaard, J., Duvall, T. L., Gough, D.O., Harvey, J W, and Rhodes, E. J.: 1985, 'Speed of Sound in he Solar Interior', Nature 315, 378-382.

Christensen-Dalsgaard, J., Däppen, W., Ajukov, S. V., et al.: 1996, 'The Current State of Solar Modeling', Science 272, 1286-1292.

Couvidat, S., Thrck-Chièze, S., and Kosovichev, A.G.: 2003, 'Solar Seismic Models and the Neutrino Prediction ;', Astrophys. J. 599, 1434-1448.

Cram, L. E., Durney, B. R., and Guenther, D. B.: 1983. 'Preliminary Observations of Velocity Fields at the Solar Poles', Astrophys. J. 267, 442-454.

Crooker, N., Joselyn, J.A., and Feynman, J. (eds.): 1997, 'Coronal Mass Ejections', American Geophysical Union, Washington DC, US.

Curdt, W., Brekke, P., Feldman, U., et al.: 2001, 'The SUMER Spectral Atlas of SolarDisk Features', Astronom. Astrophys. 375, 591-613.

Daglis, I. A. (ed.): 2001, 'Space Storms and Space Weather Hazards', NATO Science Series II: Mathematics, Physics and Chemistry 38, Kluwer, Dordrecht.

Daglis, I. A. (ed.): 2004, 'Effects of Space Weather on Technology Infrastructure', NATO Science Series II: Mathematics, Physics and Chemistry 176, Kluwer, Dordrecht.

Damé, L.: 1998, 'Low Orbit High Resolution Solar Physics with the Solar Interferometer', $A d v$. in Space Res. 21, Issue 1-2, 295-304.

Damé, L., Hersé, M., Thuillier, G., et al.: 1999, 'PICARD: Simultaneous Measurements of the Solar Diameter, Differential Rotation, Solar Constant and their Variations' $A d v$. in Space Res. 24, Issue 2, 205-214.

Danielson, R. E.: 1961, 'The Structure of Sunspot Penumbras. I. Observations', Astrophys. J. 134, 275-288.

Davis, R: 1964, 'Solar Neutrinos. II. Experimental', Phys. Rev. Left. 12, 303-05.

de Jager, C.: 2001, 'Early Solar Space Research', in J. Bleeker, J. Geiss, and M.C.E. Huber (eds.), The Century of Space Science, Kluwer Academic Publishers, Dordrecht, The Netherlands, 203-223.

Delaboudinière, J.-P., Artzner, G.E., Brunaud, J., et al. 1995 'EIT: Extreme-Ultraviolet Imaging Telescope for the SOHO Mission', Solar Phys. 162, 291-312.

Dere, K. P., Bartoe, J.-D., F., Brueckner, G. E., et al.: 1987, 'Ultraviolet Observations of Solar Fine Structure', Science 238, 1267-1269.

Deubner, F.-J.: 1975, 'Observations of Low Wavenumber Nonradial Eigenmodes of the Sun', Astronom. Astrophys. 44, 371-375.

Deubner, F-J. and Gough, D.: 1984, 'Helioseismology: Oscillations as a Diagnostic of the Solar Interior', Ann. Rev. Astronom. Astrophys. 22, 593-619.

Dulk G. A.: 1985, 'Radio Emission from the Sun and Stars', Ann. Rev. Astronom. Astrophys. 23, 169-224.

Dungey J. W.: 1958, Cosmic Electrodynamics, Cambridge University Press. 
Dupree, A. K., Goldberg, L.: 1967, 'Solar Abundance Determination from Ultraviolet Emission Lines', Solar Phys. 1, 229-241.

Duvall, T. L., Jr., Dziembowski, W.A, Goode, P.R., et al.: 1984, 'Internal Rotation of the Sun', Nature 310, 22-25.

Duvall, T. L., Jr., Harvey, J. W., and Pomerantz, M.A.: 1986, 'Latitude and Depth Variation of Solar Rotation', Nature 321, 500-501.

Duvall, T. L., Jr., Jefferies, S. M., Harvey, J. W., and Pomerantz, M. A.: 1993, 'Time-Distance Helioseismology', Nature 362, 430-432.

Duvall, F.-J., Gizon, D.: 2000, 'Time-Distance Helioseismology with f-Modes as a Method for Measurement of Near-Surface Flows', Solar Phys. 192, 177-191.

Duvall, T. L., Jr., Kosovichev, A. G.: 2001, 'New Developments in Local Area Helioseismology' in P. Brekke, B. Fleck, and J. B. Gurman (eds.), Recent Insights into the Physics of the Sun and Heliosphere: Highlights from SOHO and Other Space Missions, 159166.

Dziembowski, W. A., Goode, P. R., and Libbrecht, K. G.: 1989, 'The Radial Gradient in the Sun's Rotation', Astrophys. J. Lett. 337, L53-L57.

Dwivedi, B. N., Mohan, A, and Wilhelm, K.: 2003, 'Vacuum-Ultraviolet Emission Line Diagnostics for Solar Plasmas', in B. N. Dwivedi (ed.), Dynamic Sun, Cambridge University Press, $353-373$.

Eddy, J. A,: 1976, 'The Maunder Minimum', Science 192,1189-1202.

Eff-Darwich, A and Korzennik, S. G.: 1998, 'The Rotation of the Solar Core: Compatibility of the Different Data Sets Available', in S.G. Korzennik and A Wilson (eds.), Structure and Dynamics of the Interior of the Sun and Sun-like Stars, Proc. SOHO 6/GONG 98 Workshop, 685-692.

Eff-Darwich, A, Korzennik, S. G., and Jiménez-Reyes, S. J.: 2002, 'Inversion of the Internal Solar Rotation Rate', Astrophys. J. 573, 857-863.

Elsworth, Y., Howe, R., Isaak, G. R., et aI.: 1995, 'Slow Rotation of the Sun's Interior', Nature 376, 669.

Elwert, G.: 1961, 'Theory of X-Ray Emission of the Sun', J. Geophys. Res. 66, 391.

Evershed, J.: 1909, 'Radial Movement in Sun-Spots', Mon. Not. Roy. Astronom. Soc. 69, 454-457.

Feldman, U., Damrnasch, I. E., Wilhelm, K., et al.: 2003, 'Images of the Solar Upper Atmosphere from SUMER on SOHO', ESA SP-1274, ed.: Bruce Battrick, ESA Publications Division, Noordwijk, The Netherlands.

Fleck, B., Domingo, V., and Poland, A. (eds.): 1995, 'The SOHO Mission', Kluwer Academic Publishers. Dordrecht, The Netherlands.

Fleck, B.: 2002, 'Prospects of Future Solar Space Missions', ESA SP-505, ed.: H. SawayaLacoste, ESA Publications Division, Noordwijk, The Netherlands, 311-318.

Fleck, B. and Keller, C. U.: 2003, 'Solar Observing Facilities', in B. N. Dwivedi (ed.), Dynamic Sun, Cambridge University Press, 1089-1113.

Fontenla, J. M., Avrett, E. H. and Loeser, R: 1993, 'Energy Balance in the Solar Transition Region. III - Helium Emission in Hydrostatic, Constant-Abundance Models with Diffusion', Astrophys. J. 406, 319-345.

Forsyth, R J., Balogh, A., Smith, E. J., et al.: 1996, 'The Underlying Magnetic Field Direction in Ulysses Observations of the Southern Polar Heliosphere', Geophys. Res. Lett. 23, 3321-3324.

Fossat, E.: 1991, 'The IRIS Network for Full Disk Helioseismology - Present Status of the Programme', Solar Phys. 131, 1-12.

Foukal, P.: 1975, 'The Temperature Structure and Pressure Balance of Magnetic Loops in Active Regions', Solar Phys. 43, 327-336. 
Foukal, P., Mack, P. E., and Vernazza, J.: 1977, 'The Effect of Sunspots and Faculae on the Solar Constant', Astrophys. J. 215, 952-959.

Foukal, P.: 1978, 'Magnetic Loops, Downflows, and Convection in the Solar Corona' Astrophys. J. 223, 1046-1057.

Friis-Christensen, E., Fröhlich, C., Haigh, J. D., et al. (eds.): 2000, 'Solar Variability and Climate', Kluwer Academic Publishers, Dordrecht, The Netherlands.

Fröhlich, C. and Lean, J.: 1998, 'The Sun's Total Irradiance: Cycles, Trends, and Related Climate Change Uncertainties Since 1976', Geophys. Res. Lett. 25, 4377-4380.

Fröhlich, C.: 2000, 'Observations of Irradiance Variability', Space Sci. Rev. 94, 15-24.

Fröhlich, C.: 2003, 'Long-Term Behaviour of Space Radiometers', Metrologia 40, 60-65.

Fukuda Y., et al., Super-Kamiokande Coll.: 1998, 'Evidence for Oscillation of Atmospheric Neutrinos', Phys. Rev. Left. 81, 1562-1567.

Gabriel, A. H. and Jordan, C.: 1969, 'Interpretation of Solar Helium-like Ion Line Intensities', Mon. Not. R. Astr. Soc. 145, 241-248.

Gabriel, A. H., Grec, G., Charra, J., et al.: 1995, 'Global Oscillations at Low Frequency from the SOHO Mission (GOLF)', Solar Phys. 162, 61-99.

Geiss, J., Gloeckler, G., and von Steiger, R: 1995, 'Origin of the Solar Wind From Composition Data', Space Sci. Rev. 72, 49-60.

Giles, P. M., Duvall, T L., Jr., Scherrer, P. H., and Bogart, R. S.: 1997, 'A Flow of Material from the Sun's Equator to its Poles', Nature 390, 52.

Giordano, S., Antonucci, E., Noci, G., et al.: 2000, 'Identification of the Coronal Sources of the Fast Solar Wind', Astrophys. J. 531, L79-L82.

Giovanelli, R G.: 1947, 'Magnetic and Electric Phenomena in the Sun's Atmosphere Associated with Sunspots', Mon. Not. Roy. Astronom Soc. 107, 89-105.

Giovanelli, R G. and Jones, H. P.: 1982, 'The Three-Dimensional Structure of Atmospheric Magnetic Fields in Two Active Regions', Solar Phys. 79 267-278.

Gizon, D., Duvall, T. L., and Schou, J.: 2003, 'Wave-like Properties of Solar Supergranulation', Nature 421, 43-44.

Gold, T. and Hoyle, F.: .960, 'On the Origin of Solar Flares', Mon. Not. Roy. Astronom. Soc. 120, 89-105.

Gosling, J. T.: 1974, 'Interplanetary disturbances - classification', in: Solar wind three, Proceedings of the Third Conference, Los Angeles, University of California, 140-143.

Goldberg, L., Muller, E. A., and Aller, L. H.: 1960, 'The Abundances of the Elements in the Solar Atmosphere' Astrophys. J. Suppl. 5, 1-136.

Goode, P. R, Dziembowski, W. A., Korzennik, S. G., and Rhodes, E. J., Jr; 1991, What We Know about the Sun's Internal Rotation from Solar Oscillations', Astrophys. J. 367, 649-657.

Gopalswamy, N., Lara, A., Yashiro, S., and Howard, R. A.: 2003, 'Coronal Mass Ejections and Solar Polarity Reversal', Astrophys. J. 598, L63-L66.

Gough, D.: 1985, 'Inverting Helioseismic Data', Solar Phys. 100, 65-99.

Gough, D. and Scherrer, P. H.: 2001, 'The Solar Interior', in J. Bleeker, J. Geiss, and M.C.E. Huber (eds.), The Century of Space Science, Kluwer Academic Publishers, Dordrecht, The Netherlands, 1035-1063.

Grec, G., Fossat, E., and Pomerantz, M.: 1980, 'Solar Oscillations - Full Disk Observations from the Geographic South Pole', Nature 288, 541-544.

Grevesse, N., and Sauval, A. J..: 1998, 'Standard Solar Composition', Space Science Rev. 85, 161-174.

Gribov, V. and Pontecorvo, B.: 1969, 'Neutrino Astronomy and Lepton Charge', Phys. Lett. 28 B, 493. 
Gringauz, K.I., Bezrukikh, V. V., Balandina, S. M., et al.: 1964, 'Direct Observations of Solar Plasma Fluxes at Distances in the Order of 1, 900,000 km from the Earth 17 February 1961, and Simultaneous Observations of the Geomagnetic Field', Planet. Space Sci. 12 87-90.

Grün, E., Zook, H. A, Baguhl, M., et al.: 1993, 'Discovery of Jovian Dust Streams and Interstellar Grains by the ULYSSES Spacecraft', Nature 362, 428-430.

Güdel, M.: 2002, 'Stellar Radio Astronomy: Probing Stellar Atmospheres from Protostars to Giants', Ann. Rev. Astronom. Astrophys. 40, 217-261.

Habbal, S. R, Gloeckler, G., McNutt, R L., Jr., and Tsurutani, B. T.: 1998, 'The Solar Probe Mission: A Search for the Origin of the Solar Wind and an Unprecedented View of the Solar Surface', ESA SP-417, 83-90.

Haber, D. A, Hindman, B. W., Toomre, J., et al.: 2002, 'Solar Subsurface Weather and Possible Giant Cell Signatures', Bull. Am. Astronom. Soc. 34, 645.

Haigh, J. D.: 1996, 'The Impact of Solar Variability on Climate', Science 272, 981-984.

Haigh, J.D.: 1999, 'Modelling the Impact of Solar Variability on Climate', J. Atmos. Sol. Terr. Phys. 61, 63-72.

Haigh, J.D.: 2004, 'Climate Lectures', 34th Saas-Fee Advanced Course of the Swiss Society for Astrophysics and Astronomy,. 15-20 March 2004, Davos, Switzerland.

Hale, G.E.: 1908, 'Solar Vortices', and 'On the Probable Existence of a Magnetic Field in Sun-Spots' (2 articles), Astrophys. J. 28, 100-116, 315-343.

Hale, G.E., Seares, F.H., van Maanen, A, and Ellerman, F.: 1918, 'The General Magnetic Field of the Sun. Apparent Variation of Field-Strength with Level in the Solar Atmosphere', Astrophys. J. 47, 206-254.

Hale, G.E., Ellerman, F., Nicholson, S.B., and Joy, A. H.: 1919, 'The Magnetic Polarity of Sun-Spots', Astrophys. J. 49, 153-178.

Hale, G. E. and Nicholson, S.B.: 1925, 'The Law of Sun-spot Polarity', Astrophys. J. 62, 270-300.

Hanaoka, Y.: 1996, 'Flares and Plasma Flow Caused by Interacting Coronal Loops', Solar Phys. 165, 275-301.

Handy, B. N., Acton, L. W., Kankelborg, C. C., et al.: 1999, 'The transition region and coronal explorer', Solar Phys. 187, 229-260.

Handy, B. N. and Schrijver, C. J.: 2001, 'On the Evolution of the Solar Photospheric and Coronal Magnetic Field', Astrophys. J. 547, 1100-1108.

Harrison, R. A., Sawyer, E. C., Carter, M. K., et al.: 1995,'The Coronal Diagnostic Spectrometer for the Solar and Heliospheric Observatory', Solar Phys. 162, 233-290.

Harrison, R. A.: 1997, 'EUV Blinkers: The Significance of Variations in the Extreme Ultraviolet Quiet Sun', Solar Phys. 175, 467-485.

Harvey, J.: 1995, 'Helioseismology', Physics Today, Oct 1995, 32-38.

Harvey, J. W., Hill, F., Hubbard, R., et al.: 1996, 'The Global Oscillation Network Group (GONG) Project', Science 272, 1284-1286.

Hassler, D.M., Damrnasch, I.E., Lemaire, P., et al.: 1999, 'Solar Wind Outflow and the Chromospheric Magnetic Network', Science 283, 810-813.

Heber, B., Dröge, W, Kunow, H., et al.: 1996, 'Spatial Variation of > $106 \mathrm{MeV}$ Proton Fluxes Observed During the Ulysses Rapid Latitude Scan: Ulysses COSPIN/KET Results', Geophys. Res Lett.23, 1513-1516.

Hedin, A E., Buonsanto, M.J., Codrescu, M., et al. : 1994, 'Solar Activity Variations in Midlatitude Thermospheric Meridional Winds', J. Geophys. Res. 99, 17601-17608.

Héroux, L., Cohen, M., and Malinovsky, M.: 1972, 'The Interpretation of XUV Rocket Measurements of Intensity Ratios of Solar Spectral Lines of the Lithiumlike Ions O VI, Ne VIII, and Mg x', Sol. Phys. 23, 369-393. 
Hey, J. S., Parsons, S. J., and Phillips, J. W: 1948, 'Some Characteristics of Solar Radio Emissions' Mon. Not. Roy. Astronom. Soc. 108, 354-371.

Hill, E.: 1998, 'Solar Oscillation Ring Diagrams and Large-Scale Flows', Astrophys. J. Lett. 343, L69-L71.

Hinteregger, H. E.: 1965, 'Experimental Determination of Absolute Intensities in the Solar XUV Spectrum', Annales d'Astrophysique 28, 123-124.

Hodgson, R.: 1859, 'On a Curious Appearance Seen in the Sun', Mon. Not. Roy. Astronom. Soc. 20, 15-16.

Hovestadt, D.: 1974, 'Nuclear Composition of Solar Cosmic Rays', in: Solar wind three, Proceedings of the Third Conference, Los Angeles, University of California, 2-25.

Howe, R., Christensen-Dalsgaard, J., Hill, F, et al..: 2000, 'Dynamic Variations at the Base of the Solar Convection Zone, Science 287, 2456-2460.

Howe, R.: 2003, 'The Internal Rotation of the Sun', ESA SP-517, ed.: H. Sawaya-Lacoste, ESA Publications Division, Noordwijk, The Netherlands, 81-86.

Hoyt, D. V. and Schatten, K. H.: 1997, 'The Role of the Sun in Climate Change', Oxford University Press, New York.

Hudson, H.S.: 1991, 'Solar Flares, Microflares, Nanoflares, and Coronal Heating', Solar Phys. 133, 357-369.

Innes, D.E., Inhester, B., Axford, W.I., and Wilhelm, K.: 1997, 'Bi-Directional Plasma Jets Produced by Magnetic Reconnection on the Sun', Nature 386, 811-813.

Insley, J. E., Moore, V., and Harrison, R. A: 1997, 'First Observation of Coronal Hole Structure and Evolution Using CDS', Solar Phys. 175, 437-456.

IPCC: 2001, 'Third Assessmet Report on Climate Change', Intergovernmental Panel on Climate Change, Geneva, Switzerland.

Jacob, C.: 2002, in F Combes and D. Barret (eds.), SF2A-2002, Semaine de l' Astrophysique Francaise, Editions de Physique, Les Ulis, France.

Jordan, C.: 1972 'Identifications of Emission Lines in the EUV Solar Spectrum', Space Sci. Rev. 13, 595-606.

Jordan, C.:1980, 'The Energy Balance of the Solar Transition Region', Astronom. Astrophys. 86, 355-363.

Kirchhoff, G. and Bunser, R.: 1860, 'Chemische Analyse durch Spectralbeobachtungen', Annalen der Physik und der Chemie 110, 161-189; see also 2nd edition, 1996, Band 72, in Reprint der Bände 72 und 100, Verlag Harri Deutsch.

Kjeldseth-Moe, O.: 2003, 'The Solar Transition Region', in B.N. Dwivedi (ed.), Dynamic Sun, Cambridge University Press, 196-216.

Kohl, J. L., Esser, R., Gardler, L. D., et al.: 1995, 'The Ultraviolet Coroagraph Spectrometer for the Solar and Heliospheric Observatory', Solar Phys. 162, 313-356.

Komm, R, Howe, R., Durney, B. R., and Hill, E.: 2003, 'Temporal.. Variation of Angular Momentum in the Solar Convection Zone' Astrophys. J. 586, 650-662.

Kosovichev, A. G.: 1996, 'Tomographic Imaging of the Sun's Interior', Astrophys. J. Lett 461, L55-L57.

Kosovichev, A. G. and Duvall, T. L.: 1997, 'Acoustic Tomography of Solar Convective Flows and Structures', in F. P. Pijpers, J. Chrlstensen-Dalsgaard, and C.S. Rosenthal (eds.), SCORe'96: Solar Convection and Oscillations and their Relationship, Kluwer Academic Publishers (Astrophys. Space Sci. Lib. 225), 241-260.

Kosovichev, A. G., Duvall, T. L., and Scherrer, P. H.: 2000, 'Time-Distance Inversion Methods and Results', Solar Phys. 192, 159-176.

Krieger, A. S., Timothy, A. F., and Roelof, E. C.: 1973, 'A Coronal Hole and its Identification as the Source of a High Velocity Solar Wind Stream', Solar Phys. 29, 505-525. 
Krivova, N. A. and Solanki, S. K.: 2002, 'The 1.3-year and 156-day Periodicities in Sunspot Data: Wavelet Analysis Suggests a Common Origin', Astronom. Astrophys. 394, 701706.

Krucker, S. and , Benz, A. O. : 1998, 'Energy Distribution of Heating Processes in the Quiet Solar Corona', Astrophys. J. Lett. 501 L213-L216.

Kuin, N. P. M. and Martens, P. C. H.: 1982, 'On the Thermal Stability of Hot Coronal Loops - The Coupling Between Chromosphere and Corona', Astronom. Astrophys. 108, L1-L4.

Kumar, P., Quataert, E. J., and Bahcall, J. N.: 1996, 'Observational Searches for Solar g-Modes: Some Theoretical Considerations', Astrophys. J. Lett. 458, L83-L85.

Kundu, M. R. and White, S. M.: 2003, 'Radio Observations of Explosive Energy Releases on the Sun', in B. N. Dviwedi (ed.), Dynamic Sun, Cambridge University Press, 288313.

Landini, M. and Monsignori Fossi, B. C.: 1975, 'A Loop Model of Active Coronal Regions', Astronom. Astrophys. 42, 213-220.

Lang, K. R.: 2000, 'The Sun from Space', Springer-Verlag, Berlin, Heidelberg, New York.

Leighton R. B., Noyes, R. W., and Simon, G. W.: 1962,'Velocity Fields in the Solar Atmosphere. I. Preliminary Report', Astrophys. 1. 135, 474-500.

Leighton R. B.: 1969, 'A Magneto-Kinematic Model of the Solar Cycle', Astrophys. J. 156, $1-26$.

Lean, J., Beer, J., and Bradley, R: 1995, 'Reconstruction of Solar Irradiance Since 1610: Implications for Climate Change', Geophys. Res. Lett 22, 3195-3198.

Lean, J.: 1997, 'The Sun's Variable Atmosphere and its Relevance for Earth', Ann. Rev. Astronom. Astrophys. 35, 33-67.

Leibacher, J. W. and Stein, R. F.: 1971, 'A New Description of the Solar Five-Minute Oscillation', Astrophys. Lett 7, 191-192.

Lindsey, C. and Braun, D. C.: 1990, 'Helioseismic Imaging of Sunspots at their Antipodes', Solar Phys. 126, 101-115.

Lindsey, C. and Braun, D. C.: 2000, 'Basic Principles of Solar Acoustic Holography (Invited Review)', Solar Phys. 192, 261-284.

Lockwood, M., Stamper, R, and Wild, M.N.: 1999, 'A Doubling of the Sun's Coronal Magnetic Field During the Past 100 Years', Nature 399, 437-439.

Lockwood, M.: 2004, 'Solar Outputs, the Variations and their Effects on Earth', 34th SaasFee Advanced Course of the Swiss Society for Astrophysics and Astronomy, 15-20 March 2004, Davos, Switzerland.

Löfdahl, M. G. and Scharmer, G. B.: 2003, 'Phase Diverse Speckle Inversion Applied to Data from the Swedish 1-meter Solar Telescope', in S. L. Keil and S. V. Avakyan (eds.), Innovative Telescopes and Instrumentation for Solar Astrophysics, Proc. SPIE 4853, 567-575.

Loukitcheva, M., Solanki, S. K., Carlsson, M., and Stein, R. F.: 2004, 'Millimeter Observations and Chromospheric Dynamics', Astronom. Astrophys., 419, 747-756.

Lyot, B.: 1939, 'The Study of the Solar Corona and Prominences without Eclipses' (George Darwin Lecture, 1939), Mon. Not. Roy. Astronom. Soc. 99, 580-594.

MacQueen, R. M., Eddy, J. A., Gosling, J. T., et al.: 1974, 'The Outer Solar Corona as Observed from Skylab: Preliminary Results', Astrophys. J. 187, L85-L88.

Marsch, E., Axford, W.I., and McKenzie, J.F.: 2003, 'Solar Wind', in B.N. Dwivedi (ed.), Dynamic Sun, Cambridge University Press. 374-402.

Marsh, N. D. and Svensmark, H.: 2000, 'Low Cloud Properties Influenced by Cosmic Rays', Phys. Rev. Lettt. 85, 5004-5007. 
Masuda, S., Kosugi, T., Hara, H., et al.: 1994, 'A Loop-Top Hard X-Ray Source in a Compact Solar Flare as Evidence: for Magnetic Reconnection', Nature 371, 495.

Maunder, E. W.: 1890, 'Professor Spoerer's Researches on Sunspots', Mon. Not. Roy. Astronom. Soc. 50, 251-252.

McComas, D. J., Elliott, H. A, Schwadron, N. A, at al.: 2003, 'The Three-Dimensional Solar Wind Around Solar Maximum', Geophys. Res. Lett. 30, 24-1-24-4.

Mehltretter, J. P.: 1978, 'Balloon-borne Imagery of the Solar Granulation. II - The Lifetime of Solar Granulation', Astronom. Astrophys. 62, 311-316.

Mende, W. and Stellmacher, R: 2000, 'Solar Variability and the Search for Corresponding Climate Signals', Spac Sci. Rev. 94, 295-306.

Mikheyev, S. P. and Smirnov, A. Y.: 1985, 'Resonance Amplification of Oscillations in Matter and Spectroscopy of Solar Neutrinos', Sov. J. Nucl. Phys. 42, 913-917.

Nakariakov, V. M., Ofman, L., DeLuca, E. E., et al.: 1999, 'TRACE Observations of Damped Coronal Loop Oscillations: Implications for Coronal Heating', Science 285, 862-864.

Nakariakov, V. M. and Ofman, L.: 2001, 'Determination of the Coronal Magnetic Field by Coronal Loop Oscillations, Astronom. Astrophys. 372, L53-L56.

Nakariakov, V.M.: 2003, 'Coronal Oscillations', in B.N. Dwivedi (ed.), Dynamic Sun, Cambridge University Press, 314-332.

Narain, U. and Ulmschneider, P.: 1996, 'Chromospheric and Coronal Heating Mechanisms II', Space Sci. Rev. 75, 453-509.

Neugebauer, M. and Snyder C. W.: 1966, 'Mariner-2 Observations of the Solar Wind, 1:Average Properties', J. Geophys. Res. 71, 4469-4484.

Neugebauer, M. and von Steiger, R: 2001, 'The Solar Wind', in J. Bleeker, J. Geiss, and M.C.E. Huber (eds ), The Century of Space Science, Kluwer Academic Publishers, Dordrecht, The Netherlands, 1115'-D1140.

Nordlund, A: 2003, 'Solar Photosphere and Convection', in B. N. Dwivedi (ed.), Dynamic Sun, Cambridge University Press, 148-164.

Nolte, J. T., Krieger, A S., Timothy, A. F., et al.: 1976, 'Coronal Holes as Sources of Solar

Wind, Solar Phys. 46, 303-322.

November, L. J., Toomre, J., Gebbie, K. B., and Simon, G. W. 'The Detection of Mesogranulation on the Sun', Astrophys. J. Lett. 45, L12_126.

Parker, E. N. 1958, 'Dynamics of the Interplanetary Gas and Magnetic Fields', Astrophys. 1. 128, 664-676.

Parker, E. N.: 1979a, 'Sunspots and the Physics of Magnetic Flux Tubes. I - The General Nature of the Sunspot. II - Aerodynamic Drag', Astrophys. J. 230, 905-923.

Parker, E. N.: 1979b, 'Sunspots.and the Physics of Magnetic Flux Tubes. III - Aerodynamic Lift', Astrophys. J. 231, 250-259.

Parker, E. N.: 1979c, 'Sunspots and the Physics of Magnetic Flux Tubes V - Mutual Hydrodynamic Forces between Neighboring Tubes', Astrophys. J. 231, 270-276.

Parker, E. N.: 1983, 'Magnetic Neutral Sheets in Evolving Fields. I - General theory.II Formation of the Solar Corona', Astrophys. J. 264, 635-647 (2 papers).

Parker, E. N.: 1988, 'Nanoflares and the Solar X-Ray Corona', Astrophys. J. 330, 474-479.

Parker, E. N.: 2001, 'A History of the Solar Wind Concept', in J. Bleeker, J. Geiss, and M.C.E. Huber (eds.), The Century of Space Science, Kluwer Academic Publishers, Dordrecht, The Netherlands, 225-255.

Parnell, C. E. and Jupp, P. E.: 2000, 'Statistical Analysis of the Energy Distribution of Nanoflares in the Quiet Sun', Astrophys. J. 529, 554-569.

Pauluhn, A. and Solanki, S. K.: 2004, 'Radiance Emission by Flaring Activity', ESA SP547, ed.: H. Lacoste, ESA Publications Division, Noordwijk, The Netherlands, 323-328. 
Pasachoff, J. M. and Ladd, E. F.: 1987, 'High-Frequency Oscillations in the Corona Observed at the 1983 Eclipse', Solar Phys. 109, 365-372.

Peres, G.: 1999, 'Coronal Heating and Structuring', ESA SP-446, ed.: J.-C. Vial and B. Kaldeich-Schümann, ESA Publications Division, Noordwijk, The Netherlands, 4352.

Peter, H.: 2002, 'Hierarchy of Chromospheric Structures and their Relation to the Magnetic field', ESA SP-505, ed.: H. Sawaya-Lacoste, ESA Publications Division, Noordwijk, The Netherlands, 155-162.

Peter, H. and Judge, P. G.: 1999, 'On the Doppler shifts of Solar Ultraviolet Emission Lines', Astrophys. J. 522, 1148-1166.

Peterson, L. E. and Winckler, J. R.: 1959, 'Gamma-Ray Burst from a Solar Flare', J. Geophys. Res. 64, 697.

Petschek, H. E.: 1964, 'Magnetic Field Annihilation', in W.N. Hess (ed.), The Physics of Solar Flares, 425.

Phillips, J. L., Bame, S. J., Barnes, A., et al.: 1995, 'Ulysses Solar Wind Plasma Observations from Pole to Pole', Geophys. Res. Lett. 22, 3301-3304.

Phillips, K. J. H. and Dwivedi, B. N.: 2003, 'Probing the Sun's Hot Corona', in B. N. Dwivedi (ed.), Dynamic Sun, Cambridge University Press, 335-352.

Poletto, G., Vaiana, G. S., Zombeck, M. V., et al. 1975, 'A Comparison of Coronal X-Ray Structures of Active Regions with Magnetic Fields Computed from Photospheric Observations', Solar Phys. 44, 83-99.

Pontecorvo, B.: 1957, 'Mesonium and Antimesonium', J. Exptl. Theoret. Phys. 33, 549. [Sov. Phys. JETP 6, 429, 1958].

Pottasch, S. R.: 1963, 'The Lower Solar Corona: Interpretation of the Ultravioloet Spectrum', Astrophys. J. 137, 945-966.

Pottasch, S. R.: 1964, 'On the Chemical Composition of the Solar Corona', Mon. Not. Roy. Astronom. Soc. 128, 73-92.

Priest, E. R.: 1981, 'Theory of Loop Flows and Instability', In Solar active regions: A monograph from Skylab Solar Workshop III. Boulder, CO, Colorado Associated University Press, 213-275.

Raouafi, N.-E., Sahal-Bréchot, S., and Lemaire, P.: 2002, 'Linear Polarization of the O VI $\lambda$ 1031.92 Coronal Line. II. Constraints on the Magnetic Field and the Solar Wind Velocity Field Vectors in the Coronal Polar Holes', Astronom. Astrophys. 396, 10191028.

Raouafi, N.-E. and Solanki, S. K.: 2004, 'Effect of the Electron Density Stratification on Off-Limb O VI Line Profiles: How Large is the Velocity Distribution Anisotropy in the Solar Corona? Astronom. Astrophys. 427, 725-733.

Raymond, J. C. and Foukal, P.: 1982, 'The Thermal Structure of Solar Coronal Loops and Implications for Physical Models of Coronae', Astrophys. J. 253, 323-329.

Reale, F. and Peres, G.: 2000, 'TRACE-Derived Temperature and Emission Measure Profiles along Long-lived Coronal Loops: The Role of Filamentation', Astrophys. J. Lett. 528, L45-L48.

Reiner, M. J., Fainberg, J., and Stone, R. G.: 1995, 'Interplanetary Type III Radio Bursts Observed from High Southern Heliographic Latitudes', Space Sci. Rev. 72, 255-260.

Ren, D., Hegwer, S. L., Rimmele, T., Didkovsky, L. V., and Goode, P. R.: 2003, 'Optical Design of High-Order Adaptive Optics for the NSO Dunn Solar Telescope and the Big Bear Solar Obseratory', in S. L. Keil and S. V. Avakyan (eds.), Innovative Telescopes and Instrumentation for Solar Astrophysics, Proc. SPIE 4853, 593-599. 
Rimmele, T. R., Richards, K., Hegwer, S. L., et al.: 2003, 'Solar Adaptive Optics: a Progress Report', in P.L. Wizinowich and D. Bonaccini (eds.) Adaptive Optical System Technologies II, Proc. SPIE 4839, 635-646.

Richard, O. and Vauclair, S.: 1997, 'Local Mixing Near the Solar Core, Neutrino Fluxes and Helioseismology', Astronom. Astrophys. 322, 671-673.

Roberts, B., Edwin, P.M., and Benz, A. 0.: 1983, 'Fast Pulsations in the Solar Corona', Nature 305, 688-690.

Roelof, E. C., Gold, R. E., Simnett, G. M., et al.: 1992, 'Low-Energy Solar Electrons and Ions Observed at ULYSSES February-April, 1991 - The Inner Heliosphere as a Particle Reservoir', Geophys. Res. Lett. 19, 1243-1246.

Rosenbauer, H., Schwenn, R., Marsch, E., et al.: 1977, 'A Survey on Initial Results of the HELIOS Plasma Experiment', J. of Geophysics - Zeitschr.für Geophysik 42, 561-580.

Rosner, R., Tucker, W. H., and Vaiana, G. S.: 1978, 'Dynamics of the Quiescent Solar Corona', Astrophys. J. 220, 643-665.

Rottman, G. J., Barth, C. A., Thomas, R. J., et al.: 1982, 'Solar Spectral Irradiance, 120 to 190 nm, October 13, 1981 - January 3, 1982', Geophys. Res. Lett. 9, 587-590.

Rottman, G., Woods, T., Snow, M., and Detoma, G.: 2001, 'The Solar Cycle Variation in Ultraviolet Irradiance', Adv. in Space Res. 27(12) 1927-1932.

Russell, H. N.: 1929, 'On the Composition of the Sun's Atmosphere', Astrophys. J. 70, 1182.

Sakao, T., Kosugi, T., Masuda, S., et al.: 1994, 'Particle Acceleration in the 15 November, 1991 Solar Flare Observed with HXT', in Y. Uchida, T. Watanabe, K. Shibata, and H. S. Hudson (eds.) X-ray solar physics from Yohkoh, Frontiers Science Series, Universal Academy Press, 91.

Salby, M. L.: 1996, 'Fundamentals of Atmospheric Physics', Intemat. Geophys. Series, Vol. 6, Academic Press.

Scharmer, G. B., Gudiksen, B. V., Kiselman, D., et al.: 2002, 'Dark Cores in Sunspot Penumbral filaments', Nature 420, 151-153.

Schatzmann, E.: 1949, 'The Heating of the Solar Corona and Chromosphere', Annales d'Astrophysique 12, 203-218.

Schmieder, B. and Mein, N.: 1980, 'Mechanical Flux in the Solar Chromosphere. II Determination of the Mechanical Flux', Astronom. Astrophys. 84, 99-105.

Schrijver, C. J., Title, A. M., Harvey, K. L., et al.: 1998, 'Large-Scale Coronal Heating by the Small-Scale Magnetic Field of the Sun', Nature 394, 152-154.

Schrijver, C. J., Title, A. M., Berger, T. E., et al.: 1999, 'A New View of the Solar Outer Atmosphere by the Transition Region and Coronal Explorer', Solar Phys. 187, 261-302.

Schrijver, C. J., Zwaan, C.: 2000, 'Solar and Stellar Magnetic Activity', Cambridge University Press, New York (Cambridge astrophysics series, 34).

Schrijver, C. J., Aschwanden, M. J., and Title, A. M.: 2000, 'Transverse Oscillations in Coronal Loops Observed with TRACE - I. An Overview of Events, Movies, and a Discussion of Common Properties and Required Conditions', Solar Phys. 206, 69-98.

Schou, J., Antia, H. M., Basu, S., et al.: 1998, 'Helioseismic Studies of Differential Rotation in the Solar Envelope by the Solar Oscillations Investigation Using the Michelson Doppler Imager', Astrophys. J. 505, 390-417.

Schwabe, H.: 1838, Astr. Nachr. 15, 243.

Schwarzchild, M.: 1949, 'On Noise Arising from the Solar Granulation', Astrophys. J. $107,1-5$.

Secchi, A.: 1870, 'Le Soleil', Gauthiers-Villars, Paris.

Sheeley, N. R., Bohlin, J. D., Brueckner, G. E., et al.: 1975, 'Coronal Changes Associated with a Disappearing Filament', Solar Phys. 45, 377-392. 
Sheeley, N. R. and Golub, L.: 1979, 'Rapid Changes in the fine Structure of a Coronal 'Bright Point' and a Small Coronal 'Active Region" Solar Phys. 63, 119-126.

Sheeley, N. R.,Jr., Wang, Y.-M., Hawley, S. H., et al.: 1997, 'Measurements of Flow Speeds in the Corona between 2 and $30 R_{\odot}$ Astrophys. J. 484, 472-478.

Sheeley, N. R.,Jr., Howard, R. A., Michels, D. J.: 1985, 'Coronal Mass Ejections and Interplanetary Shocks', J. Geophys. Res. 90, 163-175.

Shibata, K., Ishido, Y., Acton, L. W, et al.: 1993, 'X-Ray Jets in the Solar Corona: Observations with YOHKOH Soft X Ray Telescope', Fourth International Toki Conference on Plasma Physics and Controlled Nuclear Fusion, 207-210.

Shimizu, T.: 1995, 'Energetics and Occurrence Rate of Active-Region Transient Brightenings and Implications for the Heating of the Active-Region Corona' Publ. Astronom. Soc. Japan 47, 251-263.

Shimizu, T., Tsuneta, S., Acton, L. W, et al.: 1992, 'Transient Brightenings in Active Regions Observed by the Soft X-ray Telescope on YOHKOH', Publ. Astronom. Soc. Japan 44, L147-L153.

Simon, G. W. and Leighton, R. B.: 1964, 'Velocity Fields in the Solar Atmosphere. III. Large Scale Motions, the Chromospheric Network, and Magnetic Fields', Astrophys. $J$. 140, $1120-1147$

Simon, G. W. and Weiss, N.O.: 1968, 'Supergranules and the Hydrogen Convection Zone', Zeitschrift für Astrophysik 69, 435-450.

Simpson, J. A:, Connell, 1. 1., Lopate, C, et al.: 1995, 'The Latitude Gradients of Galactic Cosmic Ray and Anomalous Helium Fluxes Measured on Ulysses from the Sun's South Polar Region to the Equator', Geophys. Res. Lell. 22, 3337-3340.

Skumanich, A., Smythe, C, and Frazier, E. N.: 1975, 'On the Statistical Description of Inho-mogeneities in the Quiet Solar Atmosphere. I - Linear Regression Analysis and Absolute Calibration of Multichannel Observations of the $\mathrm{Ca}^{+}$Emission Network', Astrophys. J. 200, 747-764.

Smith, E. J. and Balogh, A.: 1995, 'Ulysses Observations of the Radial Magnetic Field', Geophys. Res. Lett. 22, 3317-3320.

Smith, E. V. P. and Gottlieb, D. M.: 1974, 'Solar Flux and its Variations', Space Science Reviews 16, 771-802.

Smith, P. L. and Huber, M. C .E.: 2002, 'Spectroradiometry for Solar Physics in Space', in A. Pauluhn. M.C.E. Huber, and R. von Steiger (eds.), ISSI Sci. Rep. SR-002, 21-36.

Snodgrass H.B.: 1983, 'Magnetic Rotation of the Solar Photosphere', Astrophys. J. 270, 288-299.

Snodgrass H. B and Howard, R.: 1984, 'Limits on Photospheric Doppler Signatures for Solar Giant Cells', Astrophys. J. 284, 848-855.

SNO collaboration: 2001, 'Measurement of the Rate of $v_{\mathrm{e}}+\mathrm{d} \rightarrow \mathrm{p}+\mathrm{p}+\mathrm{e}^{-}$Interactions Produced by ${ }^{8}$ B Solar Neutrinos at the Sudbury Neutrino Observatory' Phys. Rev. Lett 87, 071301 .

SNO collaboration: 2002, 'Measurement of Day and Night Neutrino Energy Spectra at SNO and Constraints on Neutrino Mixing Parameters', Phys. Rev. Lett. 89, 011302.

Solanki, S. K., Livingstone, W., and Ayres, T.: 1994, 'New Light on the Heart of Darkness of the Solar Chromosphere', Science 263, 64-66.

Solanki, S. K. and Fligge, M.: 2000, 'Reconstruction of Past Solar Irradiance', Space Sci. Rev. 94, 127-138.

Solanki, S. K. and Hammer, R.: 2001, 'The Solar Atmosphere', in J. Bleeker, J. Geiss, and M.C.E. Huber (eds.), The Century of Space Science, Kluwer Academic Publishers, Dordrecht. The Netherlands, 1065-1088. 
Solanki, S. K.: 2002, 'Solar Variability', in A. Pauluhn, M. C. E. Huber, and R. von Steiger (eds.), ISSI Sci. Rep. SR-002, 1-20.

Solanki, S. K.: 2003, 'Sunspots: An Overview', Astronom. Astrophys. Rev. 11, 153-286.

Solanki, S. K., Lagg, A., Woch, J., et al.: 2003, 'Three-Dimensional Magnetic Field Topology in a Region of Solar Coronal Heating', Nature 425, 692-695.

Solanki, S. K. and Krivova, N. A.: 2003, 'Can Solar Variability Explain Global Warming Since 1970' J. Geophys. Res. 108(A5), SSH 7-1.

Spörer, E. W. G: 1887, Über die Periodizität der Sonnenflecken seit dem Jahre 1618', Vierteljahrsschr. Astron. Ges. Leipzig 22, 323-329.

Spruit, H. C., Nordlund, A., and Title, A. M.: 1990, 'Solar Convection', Ann. Rev. Astronom. Astrophys. 28, 263-301.

von Steiger, R., Schwadron, N. A, Fisk, L. A., et a1.: 2000, 'Composition of Quasi-Stationary Solar Wind Flows from 1 Ulysses/Solar Wind Ion Composition Spectrometer', J. Geophys. Res. 105, 27217-27238.

Stein, R. E.: 1982, 'On the Modal Structure of the Solar Oscillations', Astronom. Astrophys. $105,417-418$.

Stenfl J. O.: 1973, 'Magnetic-Field Structure of the Photospheric Network', Solar Phys. 32, 41-63.

Stenflo, J. O., Keller, C. U., and Gandorfer, A: 1998, 'Differential Hanle Effect and the Spatial Variation of Turbulent Magnetic Fields on the Sun', Astronom. Astrophys. 329, 319-328.

Stix, M.: 2002, 'The Sun', 2nd edition, Springer-Verlag, Berlin, Heidelberg, New York.

Stucki, K., Solanki, S. K., Pike, C. D., et al.: 2002, 'Properties of Ultraviolet Lines Observed with the Coronal Diagnostic Spectrometer (CDS/SOHO) in Coronal Holes and the Quiet Sun', Astronom. Astrophys. 381, 653-667.

Stuiver, M.: 1961, 'Variations in Radiocarbon Concentration and Sunspot Activity', J. Geophys. Res. 66, 273

Sun, M., Chou, D., and the TON Team: 2003, 'The Progress of the Taiwan Oscillation Network Project', Space Sci. Rev. 107, 103-106.

Švestka: 1976, 'Optical Observations of Flares', Philosoph. Trans., Series A 281 (1304) 435441

Švestka, Z.: 2003, 'Solar Activity’, in B. N. Dwivedi (ed.), Dynamic Sun, Cambridge University Press, 237-261

Sweet, P. A: 1969, 'Mechanisms of Solar Flares', Ann. Rev. Astronom. Astrophys. 7, 149176.

Thompson M. J.: 1990, 'A New Inversion of Solar Rotational Splitting Data', Solar Phys: $125,1-12$.

Thompson, M. J., Christensen-Dalsgaard, J., Miesch, M. S., and Toomre J.: 2003, 'The Internal Rotation of the Sun', Ann. Rev. Astronom. Astrophys. 41, 599-643.

Timothy, A. E., Krieger, P. S., and Vaiana, G. S.: 1975, 'The Structure and Evolution of Coronal Holes', Solar Phys. 42, 135-156.

Title, A M., Tarbell, T. D., Topka, K. P., et al.: 1989, 'Statistical Properties of Solar Granulation Derived from the SOUP Instrument on Spacelab 2', Astrophys. J. 336, 475-494.

Tomczyk, S., Streander, K , Card, G., et al.: 1995, 'An Instrument to Observe Low-Degree Solar Oscillations', Solar Phys. 159, 1-21.

Toner, C. G., Jefferies, S. M., and Toutain, T.: 1999, 'Increasing the Visibility of Solar Oscillations', Astrophys. J. 518, L127-L130.

Toomre, J., Christensen-Dalsgaard, J., Hill, F., et al.: 2003, 'Transient Oscillations near the Solar Tachocline', ESA SP-517, ed.: H. Sawaya-Lacoste, ESA Publications Division, Noordwijk, The Netherlands, 409-412. 
Tousey, R: 1953, 'Solar Work at High Altitudes from Rockets', in G. P. Kuiper (ed.), The Sun, University of Chicago Press, 658-676.

Tsuneta, S., Takahashi, T., Acton, L. W., et a1.: 1992, 'Global Restructuring of the Coronal Magnetic Fields Observed with the YOHKOH Soft X-ray Telescope', Publ. Astronom. Soc. Japan 44, L211-L214.

Tsuneta, S.: 1997, 'Moving Plasmoid and Formation of the Neutral Sheet in a Solar Flare' Astrophys. J. 483, 507-514.

Turck-Chièze, S., Basu, S., Berthomieu, G., et al.: 1998, 'Sensitivity of the Sound Speed to the Physical Processes included in the Standard Solar Model', in S. G. Korzennik and A. Wilson (eds.), Structure and Dynamics of the Interior of the Sun and Sun-like Stars, Proc. SOHO 6/GONG 98 Workshop, 555-560.

Turck-Chièze, S., Couvidat, S., Kosovichev, A. G., et al.: 2001a, 'Solar Neutrino Emission Deduced from a Seismic Model', Astrophys. J Lett. 555, L66-L73.

Turck-Chièze, S., Robillot, J.M., Dzitko, H., et al: 2001b, 'G-Mode: a New Generation of Helioseismic Instrument', ESA SP-464, ed.: A. Wilson, ESA Publications Division, Noordwijk, The Netherlands, 331-335.

Turck-Chièze, S., García, R. A., Couvidat, S., et al.: 2004, 'Looking for Gravity-Mode Multiplets with the GOLF Experiment aboard SOHO', Astrophys. J. 604, 455-468.

Ulmschneider, P. and Kalkofen, W.: 2003, 'Heating of the Solar Chromosphere', in B. N. Dviwedi (ed.), Dynamic Sun, Cambridge University Press, 181-195.

Ulrich, R. K.: 1970, 'The Five-Minute Oscillations on the Solar Surface', Astrophys. J. 162, 993-1002.

Ushida, Y.: 1970, Publ. Astronom. Soc. Japan 22, 41.

Usoskin, I. G., Solanki, S. K., Schüssler, M., et al.: 2003, 'Millennium-Scale Sunspot Number Reconstruction: Evidence for an Unusually Active Sun Since the 194Os', Phys. Rev. Lett. 91, 211101.

Usoskin, I. G., Mursula, K., Solanki, S. K., et al.: 2004, 'Reconstruction of Solar Activity for the Last Millennium Using ${ }^{10} \mathrm{Be}$ data', Astronom. Astrophys. 413, 745-751.

Vaiana, G. S., Davis, J. M., Giacconi, R, et al.: 1973, 'X-Ray Observations of Characteristic Structures and Time Variations from the Solar Corona: Preliminary Results from SKYLAB', Astrophys. J., 185, L47-L51.

Vaiana, G. S. and Rosner, R.: 1978, 'Recent Advances in Coronal Physics', Ann. Rev. Astronom. Astrophys. 16, 393-428.

Vauclair, S.: 1998, 'Element Settling in the Solar Interior', Space Science Rev., 85, 71-78.

Vernazza, J. E., Avrett, E. H., and Loeser, R: 1973, 'Structure of the Solar Chromosphere. I. Basic Computations and Summary of the Results', Astrophys. J. 184, 605-632.

Vernazza, J. E., Avrett, E. H., and Loeser, R: 1981, 'Structure of the Solar Chromosphere. III. Models of the EUV Brightness Components of the Quiet Sun', Astrophys. J. Suppl. Series 45, 635-725.

Vernazza, J. E. and Reeves, E. M.: 1978,'Extreme Ultraviolet Composite Spectra of Representative Solar Features' Astrophys J. Suppl. Ser. 37, 485-513.

Waldmeier, M.: 1957, 'Die Sonnenkorona II', Verlag Birkhäuser, Basel/Stuttgart.

Waldmeier, M.: 1975, 'The Coronal Hole at the 7 March 1970 Solar Eclipse', Solar Phys. 40, 351-358.

Walker, A. B. C., Jr.: 1972, The Coronal X-Spectrum: Problems and Prospects, Space Sci. Rev. 13, 672-730.

Wang, Y.-M., Nash. A.G., and Sheeley, N.R, Jr.: 1989, 'Magnetic Flux Transport on the Sun, Science 245,712-718. 
Wang, T., Solanki, S. K., Curdt, W., et al.: 2002, 'Doppler Shift Oscillations of Hot Solar Coronal Plasma Seen by SUMER: A Signature of Loop Oscillations?', Astrophys. J. 574, L101-L104.

Wang, T., Solanki, S. K., Curdt, W., et al.: 2003, 'Hot Coronal Loop Oscillations Observed with SUMER: Examples and Statistics', Astronam. Astrophys. 406 1105-1121.

White, O. R. (ed.): 1977, The Solar Output and its Variation, Proceedings of a Workshop, held in Boulder, Colorado, April 26-28, 1976, Associated University Press.

White, S., Lee, J., Aschwanden, M. A., and Bastian, T. S.: 2003, 'Imaging Capabilities of the Frequency Agile Solar Radiotelescope (FASR)', in S. L. Keil and S. V. Avakyan (eds.), Innovative Telescopes and Instrumentation for Solar Astrophysics, Proc. SPIE 4853, 531-541.

Wild J. P.: 1950, Aust. J. Sci. Res. A 3, 399.

Wilhelm, K., Curdt, W., Marsch, E., et al.: 1995, 'SUMER - Solar Ultraviolet Measurements of Emitted Radiation', Solar Phys. 162, 189-231

Wilhelm, K, Marsch, E., Dwivedi, B. N., et al.: 1998, 'The Solar Corona Above Polar Coronal Holes as Seen by SUMER on SOHO', Astrophys. J. 500, 1023-1038.

Wilhelm, K., Dammasch, I. E., Marsch, E., and Hassler, D. M.: 2000, 'On the Sources Regions of the Fast Solar WInd n Polar Coronal Holes', Astronom. Astrophys. 353, $749-756$.

Wilhelm, K. and Kalkofen, W.: 2003, 'Observations of the Upper Solar Chromosphere with SUMER', Astronom. A 1trophys. 408, 1137-1154.

Williams, D. R., Mathoudiakis, M., Gallagher, P. T., et al.: 2002, 'An Observational Study of Magneto-Acoustic Wave in the Solar Corona', Mon. Not. Roy. Astronom. Soc. 336, $747-752$.

Withbroe, G. L. and Noyes, R. W.: 1977, 'Mass and Energy Flow in the Solar Chromosphere' and Corona', Ann. Rev. Astronom. Astrophys. 15, 363-387.

Withbroe, G. L.: 1981, 'Physics of Static Loop Structures and Scaling Laws', In Solar active regions: A monograph from Skylab Solar Workshop III. Boulder, CO, Colorado Associated University Press, 199-212.

Wolfenstein, L.: 1978, 'Neutrino Oscillations in Matter', Phys. Rev. D 17, 2369-2374.

Zhao, J., Kosovichev, A. G., and Duvall, T. L Jr.: 2001, 'Investigation of mass Flows beneath a Sunspot by Time-Distance Helioseismology' Astrophys. J. 557, 384-388.

Zhitnik, I. A., Bugaenko, O. I., Ignat'ev, A. P., et al.: 2003, 'Dynamic 10 MK Plasma Structures' Observed in Monochromatic Full-Sun Images by the SPIRIT Spectroheliograph on the CORONAS-F Mission', Mon. Not. Roy. Astronom. Soc. 338, 67-71

Zirin, H.: 1966, 'The Solar Atmosphere', Blaisdell, Waltham, Mass., USA.

Zirker, J. B.: 1971 'Coronal Holes and High-Speed Wind Streams', Rev. Geophys. Space Phys. 15, 257-269. 